\title{
QUADRATIC SYSTEMS WITH AN INTEGRABLE SADDLE: A COMPLETE CLASSIFICATION IN THE COEFFICIENT SPACE $\mathbb{R}^{12}$
}

\author{
JOAN C. ARTÉS ${ }^{1}$, JAUME LLIBRE ${ }^{1}$ AND NICOLAE VULPE ${ }^{2}$
}

\begin{abstract}
A quadratic polynomial differential system can be identified with a single point of $\mathbb{R}^{12}$ through the coefficients. Using the algebraic invariant theory we classify all the quadratic polynomial differential systems of $\mathbb{R}^{12}$ having an integrable saddle. We show that there are only 47 topologically different phase portraits in the Poincaré disc associated to this family of quadratic systems up to a reversal of the sense of their orbits. Moreover each one of these 47 representatives is determined by a set of affine invariant conditions.
\end{abstract}

\section{InTRODUCTION AND STATEMENT OF MAIN RESUltS}

Let $\mathbb{R}[x, y]$ be the ring of the polynomials in the variables $x$ and $y$ with coefficients in $\mathbb{R}$. We consider a system of polynomial differential equations or simply a polynomial differential system in $\mathbb{R}^{2}$ defined by

$$
\begin{aligned}
& \dot{x}=P(x, y), \\
& \dot{y}=Q(x, y),
\end{aligned}
$$

where $P, Q \in \mathbb{R}[x, y]$. We say that the maximum of the degrees of the polynomials $P$ and $Q$ is the degree of system (1). A quadratic polynomial differential system or simply a quadratic system $(Q S)$ is a polynomial differential system of degree 2 . We say that a quadratic system (1) is non-degenerate if the polynomials $P$ and $Q$ are relatively prime or coprime.

In [20] H. Poincaré defined the notion of a center for a real polynomial differential system in the plane (i.e. an isolated singularity surrounded by a continuum of periodic orbits). The analysis of the limit cycles which bifurcate from a focus or a center of a quadratic system was made by Bautin [8], by providing the structure of the power series development of the displacement function defined near a focus or a center of a quadratic system. More recently the structure of this displacement function has been understand for any weak focus of a polynomial differential system. More precisely, first by using a linear change of coordinates and a rescaling of the independent variable, we transform any polynomial differential system having a focus or a center at the origin with purely imaginary eigenvalues (i.e. having a weak focus) into the form

$$
\begin{aligned}
& \dot{x}=y+P(x, y), \\
& \dot{y}=-x+Q(x, y),
\end{aligned}
$$

where $P$ and $Q$ are polynomials without constant and linear terms. Then the return map $x \mapsto h(x)$ defined for $|x|<R$, where $R$ is a positive number which is sufficiently small to insure that the power series expansion of $h(x)$ at the origin is convergent. Of course, limit cycles correspond to isolated zeros of the displacement function $d(x)=h(x)-x$. The structure of the power series for the displacement function is given by the following restatement of Bautin's fundamental result (see [23] for more details): There exists a positive integer $m$ and a real number $R>0$ such that the displacement function for the polynomial differential system (2) can be written as

$$
d(x)=\sum_{j=1}^{m} v_{2 j+1} x^{2 j+1}\left[\alpha_{0}+\sum_{k=1}^{\infty} \alpha_{k}^{2 j+1} x^{k}\right]
$$

Date: 25 May, 2010.

1991 Mathematics Subject Classification. Primary 34C05, 34A34.

Key words and phrases. quadratic vector fields, weak saddle, type of singularity, 
for $|x|<R$, where the $v_{2 j+1}$ 's and the $\alpha_{k}^{2 j+1}$ 's are homogeneous polynomials in the coefficients of the polynomials $P$ and $Q$.

The constants $V_{j}=v_{2 j+1}$ are called the focus quantities or the Poincaré-Liapunov constants. A weak focus for which $V_{1}=\ldots=V_{n-1}=0$ and $V_{n} \neq 0$ is a weak focus of order $n$. If all the focus quantities are zero then the weak focus is a center. Note that any weak focus has finitely many focus quantities, in our notation exactly $m$.

It is known that a polynomial differential system (2) has a center at the origin if and only if there exists a local analytic first integral of the form $H=x^{2}+y^{2}+F(x, y)$ defined in a neighborhood of the origin, where $F$ starts with terms of order higher than 2 . This result is due to Poincaré [21] and Liapunov [15], see also Moussu [17].

A weak saddle is a hyperbolic saddle such that the trace of its linear part is zero. We consider now a polynomial differential system in the plane having a weak saddle at the origin. In a sufficiently small neighborhood of the origin, the stable and unstable manifolds, $W^{s}$ and on $W^{u}$, determine four regions. We denote by $R$ the closure of one of these regions. Let $S$ and $U$ be cross sections of the flow inside $R$, with one endpoint on $W^{s}$ and $W^{u}$ respectively, parameterized respectively by $s \geq 0$ and $u \geq 0$, the points $s=0$ and $u=0$ lying respectively on $W^{s}$ and on $W^{u}$

Let $F: S \rightarrow U$ be the Poincaré map from $S$ to $U$ following the flow near the saddle. The saddle quantity or the dual Poincaré-Liapunov constant of order $n$ is

$$
L_{n}=\lim _{s \rightarrow 0}-\frac{F^{(n+1)}(s)}{(n+1) ! \log s} .
$$

A saddle point with zero trace for which $L_{1}=\ldots=L_{n-1}=0$ and $L_{n} \neq 0$ is a weak saddle of order $n$. If all the saddle quantities are zero then we say that the weak saddle is an integrable saddle. Of course the saddle quantities are independent of the choice of $R, S$ and $U$, and are invariant under changes of coordinates on $S$ and $U$.

The notion of weak saddle was introduced by Cai Suilin [10] for the quadratic systems, and generalized to any polynomial differential system by Joyal and Rousseau [14], see these papers for additional information.

Doing a linear change of coordinates and a rescaling of the independent variable, any polynomial differential system having a weak saddle at the origin can be written as

$$
\begin{aligned}
& \dot{x}=y+p(x, y), \\
& \dot{y}=x+q(x, y),
\end{aligned}
$$

where $p$ and $q$ are polynomials without constant and linear terms. Doing the change of variables $x=(\bar{w}+w) / 2$ and $y=(\bar{w}-w) i / 2$ the differential system (3) becomes

$$
\begin{aligned}
& \dot{w}=i w+P(w, \bar{w}), \\
& \dot{\bar{w}}=-i \bar{w}+Q(w, \bar{w}),
\end{aligned}
$$

Then the focus quantities $V_{j}$ of system (4) coincide with the saddle quantities $L_{j}$ of system (3). Due to this duality between focus quantities and saddle quantities it follows that every weak saddle has finitely many saddle quantities, and that an integrable saddle has an analytic first integral defined in a neighborhood of it.

If a quadratic system possesses a weak saddle according to [10] (see also, [14]) via an affine transformation this system can be written as

$$
\dot{x}=x+a x^{2}+b x y+c y^{2}, \quad \dot{y}=-y-d x^{2}-l x y-m y^{2},
$$

with the weak saddle at the origin. For these systems Cai Sui Lin has calculated the first three saddle quantities

$$
\begin{aligned}
& L_{1}^{*}=l m-a b, \\
& L_{2}^{*}=k b(2 m-b)(m+2 b)-c l(2 a-l)(a+2 l), \\
& L_{3}^{*}=(c k-l b)[\operatorname{acl}(2 a-l)-b k m(2 m-b)] .
\end{aligned}
$$

Then the weak saddle $(0,0)$ is of the first (respectively second; third) order if $L_{1}^{*} \neq 0$ (respectively $\left.L_{1}^{*}=0, L_{2}^{*} \neq 0 ; L_{1}^{*}=L_{2}^{*}=0, L_{3}^{*} \neq 0\right)$ and it is an integrable saddle if $L_{1}^{*}=L_{2}^{*}=L_{3}^{*}=0$. 
During the last one hundred years quadratic vector fields have been investigated intensively as one of the easiest but not trivial families of nonlinear differential systems, and more than one thousand papers have been published about these vectors fields (see for instance [22, 34, 33]), but the problem of classifying all the quadratic vector fields (even integrable ones) remains open. For more information on the integrable differential vector fields in dimension 2, see for instance [11].

The objective of this paper is to classify all the global phase portraits of the quadratic systems having an integrable saddle. Of course this problem is algebraically solvable in the sense indicated by Coppel [12], because the classification of the quadratic centers is algebraically solvable.

Two quadratic systems are topologically equivalent if there is a homeomorphism on the Poincaré disc sending orbits of one to orbits of the other, preserving or reversing simultaneously the sense of all the orbits.

Through the coefficients of a quadratic system every one of these systems can be identified with a single point of $\mathbb{R}^{12}$. Using the invariant theory we classify all the quadratic polynomial differential systems of $\mathbb{R}^{12}$ having an integrable saddle. More precisely we prove that there are only 47 topologically different phase portraits in the Poincaré disc associated to this family of quadratic systems up to a reversal of the sense of their orbits (see the Main Theorem). Moreover each one of these 47 representatives is determined by a set of invariant conditions (see Tables 1, 2 and 3).

The first step in a systematic study of the subclasses of $Q S$ was achieved in the determining the subclass $Q C$ of all systems in $Q S$ which have a center. The phase portraits of this class were given by $\mathrm{N}$. Vulpe in [31] and are here denoted by $V u l_{\#}$ using his classification. There are 31 cases.

Notice that the duality between focus and saddle quantities does not extend to the number of phase portraits since we have 31 in one case and 47 in the other.

Theorem 1 (Main Theorem). Consider a non-degenerate quadratic system of differential equations.

(i) This system possesses an integrable saddle and the configuration of all its singularities (finite and infinite) is given in Tables 1 and 2 if and only if the corresponding conditions given by these tables hold.

(ii) The phase portrait of this system corresponds to the one of 48 portraits given by Table 3 and either it is determined univocally by the respective configuration, or it is determined by the configuration and additional conditions as indicated in Table 3. Moreover there are 47 topologically distinct phase portraits (Portraits 30 and 31 are topologically equivalent, one has a focus and the other in its place has a node).

Remark 1. Hamiltonian systems correspond to cases 43-55. However there are cases with integrable saddle whose phase portrait is topologically equivalent to Hamiltonian even the system is not Hamiltonian. We denote these cases in Table 3 with the symbol $\cong$.

We note that the planar polynomial differential systems can have centers where both eigenvalues are zero but its linear part is not identically zero (nilpotent centers), or centers having its linear part identically zero. But it is well-known that all quadratic centers have eigenvalues of the form $\pm \omega i$ with $\omega \neq 0$. A singular point is hyperbolic if the real part of its eigenvalues is not zero.

On the other hand quadratic systems can have singular points which are topologically equivalent to hyperbolic saddles (singular points whose neighborhood is formed by four hyperbolic sectors) which are not hyperbolic but can also be considered "weak" in the sense that they have the trace of its linear part zero (nilpotent saddles, see [13]). The order of weakness of this new kind of "weak saddles" has not been studied for the moment.

The work is organized as follows. In Section 2 we give some preliminary results including the definitions of singular points, a brief description of the Poincaré compactification, the notation used in Tables 1, 2 and 3 and in Figure 1, an important theorem needed for this paper about 
weak singularities which is proved in [32], and a short introduction to the needed invariant theory with the definition of the invariants and $\mathrm{T}$-comitants needed for this paper.

In Section 3 we prove step by step our Main Theorem dividing the proof in two main cases $\left(b^{2}+l^{2} \neq 0\right.$ and $b=l=0$ in systems (5)) and each case splits in four different subcases (those described in Tables 1 and 2).

\begin{tabular}{|c|c|c|c|c|c|}
\hline \multicolumn{6}{|c|}{$\begin{array}{l}\text { Conditions for the existence of an integrable saddle [statement (b) of Theorem 2]: } \\
\qquad \mathcal{T}_{3} \neq 0, \mathcal{T}_{4}=\mathcal{F}_{1}=\mathcal{F}_{2}=\mathcal{F}_{3} \mathcal{F}_{4}=0, \mathcal{T}_{3} \mathcal{F}>0\end{array}$} \\
\hline \multicolumn{4}{|c|}{ Additional conditions for configurations } & $\begin{array}{l}\text { Configuration } \\
\text { of singularities }\end{array}$ & No. \\
\hline \multicolumn{4}{|c|}{$\mu_{0}<0$} & $\$, n, n, n ; S, S, N$ & 1 \\
\hline \multirow{4}{*}{$\mu_{0}>0$} & \multicolumn{3}{|c|}{$\mathbf{D}<0$} & $\$, s, n, n ; S, N, N$ & 2 \\
\hline & \multirow{2}{*}{$\mathbf{D}>0$} & \multicolumn{2}{|c|}{$W_{4}<0$} & $\$, f ; N$ & 3 \\
\hline & & \multicolumn{2}{|c|}{$W_{4} \geq 0$} & $\$, n ; N$ & 4 \\
\hline & \multicolumn{3}{|c|}{$\mathbf{D}=0$} & $\$, n, \overline{s n}_{(2)} ; N, \overline{\left(\begin{array}{l}0 \\
2\end{array}\right)} S N$ & 5 \\
\hline \multirow{3}{*}{$\mu_{0}=0$} & \multirow{2}{*}{$\eta<0$} & \multicolumn{2}{|c|}{$W_{4} \neq 0$} & $\$, f ; N$ & 6 \\
\hline & & \multicolumn{2}{|c|}{$W_{4}=0$} & $\$, n ; N$ & 7 \\
\hline & \multicolumn{3}{|c|}{$\eta>0$} & $c, s ; N, \overline{\left(\begin{array}{l}1 \\
1\end{array}\right)} S N, \overline{\left(\begin{array}{l}1 \\
1\end{array}\right)} S N$ & 8 \\
\hline \multicolumn{6}{|c|}{$\begin{array}{l}\text { Conditions for the existence of an integrable saddle [statement (c) of Theorem 2]: } \\
\qquad \mathcal{T}_{4}=\mathcal{T}_{3}=0, \mathcal{T}_{2} \neq 0,\left(c_{4}\right) \cup\left(c_{5}\right)\end{array}$} \\
\hline \multirow{5}{*}{$\mu_{0}<0$} & \multirow{4}{*}{$\mathbf{D}<0$} & \multicolumn{2}{|c|}{$\eta<0$} & $\$, c, n, n ; S$ & 9 \\
\hline & & \multirow{2}{*}{$\eta>0$} & $\widetilde{K}<0$ & $\$, c, s, s ; N, N, N$ & 10 \\
\hline & & & $\widetilde{K}>0$ & $\$, c, n, n ; S, S, N$ & 11 \\
\hline & & \multicolumn{2}{|c|}{$\eta=0$} & 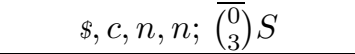 & 12 \\
\hline & \multicolumn{3}{|c|}{$\overline{\mathbf{D}}>0$} & $\$, \$ ; N, N, N$ & 13 \\
\hline \multirow{6}{*}{$\mu_{0}>0$} & \multirow{3}{*}{$\mathbf{D}<0$} & \multicolumn{2}{|c|}{$\eta<0$} & $\$, \$, n, n ; N$ & 14 \\
\hline & & \multicolumn{2}{|c|}{$\eta>0$} & $\$, \$, n, n ; S, N, N$ & 15 \\
\hline & & \multicolumn{2}{|c|}{$\eta=0$} & $\$, \$, n, n ; \overline{\left(\begin{array}{l}0 \\
3\end{array}\right)} N$ & 16 \\
\hline & \multirow{3}{*}{$\mathbf{D}>0$} & \multicolumn{2}{|c|}{$\eta<0$} & $\$, c ; N$ & 17 \\
\hline & & \multicolumn{2}{|c|}{$\eta>0$} & $\$, c ; S, N, N$ & 18 \\
\hline & & \multicolumn{2}{|c|}{$\eta=0$} & 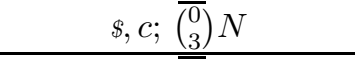 & 19 \\
\hline \multirow{5}{*}{$\mu_{0}=0$} & \multirow{2}{*}{$\eta<0$} & \multicolumn{2}{|c|}{$\widetilde{K} \neq 0$} & $\$, c ;\left(\begin{array}{l}2 \\
1\end{array}\right) N$ & 20 \\
\hline & & & & $\$, c ; N$ & 21 \\
\hline & & & $W_{4}<0$ & 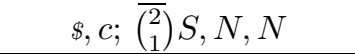 & 22 \\
\hline & $\eta>0$ & $n+u$ & $W_{4}>0$ & $\$, \$ ; \overline{\left(\begin{array}{l}2 \\
1\end{array}\right)} N, N, N$ & 23 \\
\hline & & & & 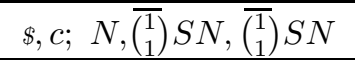 & 24 \\
\hline
\end{tabular}

TABLE 1. Configurations of the finite singularities for Quadratic systems having an integrable saddle: the case $\mathcal{T}_{3}^{2}+\mathcal{T}_{2}^{2} \neq 0$ 


\begin{tabular}{|c|c|c|c|c|c|c|}
\hline \multicolumn{7}{|c|}{$\begin{array}{l}\text { Conditions for the existence of an integrable saddle [statement }(e) \text { of Theorem 2]: } \\
\qquad \mathcal{T}_{4}=\mathcal{T}_{3}=\mathcal{T}_{2}=0, \sigma \neq 0,\left(e_{3}\right)\end{array}$} \\
\hline \multicolumn{5}{|c|}{ Additional conditions for configurations } & $\begin{array}{l}\text { Configuration } \\
\text { of singularities }\end{array}$ & No. \\
\hline \multicolumn{5}{|c|}{$\mu_{0}<0$} & $\$, \bar{s}_{(3)} ; N, N, N$ & 25 \\
\hline \multirow{3}{*}{$\mu_{0}>0$} & \multicolumn{4}{|c|}{$\eta<0$} & $\$, \widehat{e s}_{(3)} ; N$ & 26 \\
\hline & \multicolumn{4}{|c|}{$\eta>0$} & $\$, \widehat{e s}(3) ; S, N, N$ & 27 \\
\hline & \multicolumn{4}{|c|}{$\eta=0$} & $\left.\$, \widehat{e s}_{(3)} ; \overline{(}^{0}\right) N$ & 28 \\
\hline \multirow{5}{*}{$\begin{array}{l}\mu_{0}=0 \\
\mu_{1} \neq 0\end{array}$} & \multirow{4}{*}{$\mathbf{D}<0$} & & \multirow{2}{*}{$\tilde{L}<0$} & $\tilde{N} \leq 0$ & $\$, n, n ; S, \widehat{\left(\begin{array}{c}1 \\
2\end{array}\right)} P E P-H$ & 29 \\
\hline & & $C_{2} \neq 0$ & & $\widetilde{N}>0$ & $\$, n, n ; S, \widehat{\left(\begin{array}{l}1 \\
2\end{array}\right)} P E P-P H P$ & 30 \\
\hline & & & \multicolumn{2}{|c|}{$\tilde{L}>0$} & $\$, n, n ; N, \widehat{\left(\begin{array}{l}1 \\
2\end{array}\right)} H H H-H$ & 31 \\
\hline & & \multicolumn{3}{|c|}{$C_{2}=0$} & $\$, n, n ;(\infty, \emptyset)$ & 32 \\
\hline & \multicolumn{4}{|c|}{$\mathbf{D}>0$} & $\$ ; N, \widehat{\left(\begin{array}{l}1 \\
2\end{array}\right)} P E P-H$ & 33 \\
\hline \multirow{9}{*}{$\begin{array}{l}\mu_{0}=0 \\
\mu_{1}=0\end{array}$} & \multicolumn{4}{|c|}{$\mu_{2}<0$} & $\$, s ; N,\left(\begin{array}{l}2 \\
2\end{array}\right) P E P-P E P$ & 34 \\
\hline & \multirow{4}{*}{\multicolumn{2}{|c|}{$\mu_{2}>0$}} & \multicolumn{2}{|c|}{$\tilde{L}<0$} & $\$, n ; S,\left(\begin{array}{l}2 \\
2\end{array}\right) P E P-P E P$ & 35 \\
\hline & & & \multicolumn{2}{|c|}{$\tilde{L}>0$} & $\$, n ; N,\left(\begin{array}{l}2 \\
2\end{array}\right) P P H-P P H$ & 36 \\
\hline & & & \multirow{2}{*}{$\tilde{L}=0$} & $C_{2} \neq 0$ & $\$, n ;\left(\begin{array}{l}2 \\
3\end{array}\right) P E P H-P$ & 37 \\
\hline & & & & $C_{2}=0$ & $\$, n ;(\infty, N)$ & 38 \\
\hline & \multirow{4}{*}{$\mu_{2}=0$} & \multicolumn{3}{|c|}{$\widetilde{K} \neq 0$} & $\$ ; N,\left(\begin{array}{l}3 \\
2\end{array}\right) P E P-P H$ & 39 \\
\hline & & \multirow{3}{*}{$\widetilde{K}=0$} & \multicolumn{2}{|c|}{$\tilde{L} \neq 0$} & $\$ ; N,\left(\begin{array}{l}3 \\
2\end{array}\right) P E P-H$ & 40 \\
\hline & & & \multirow{2}{*}{$\tilde{L}=0$} & $\kappa_{1} \neq 0$ & 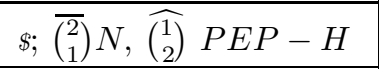 & 41 \\
\hline & & & & $\kappa_{1}=0$ & $\$ ;\left(\begin{array}{l}1 \\
1\end{array}\right) S N,\left(\begin{array}{l}2 \\
2\end{array}\right) P E P-P E P$ & 42 \\
\hline \multicolumn{7}{|c|}{$\begin{array}{l}\text { Conditions for the existence of an integrable saddle [statement }(f) \text { of Theorem 2]: } \\
\text { Hamiltonian systems } \Rightarrow \sigma=0, \cup_{1}^{6}\left(f_{j}\right)\end{array}$} \\
\hline \multirow{4}{*}{\multicolumn{2}{|c|}{$\mu_{0}<0$}} & \multicolumn{3}{|c|}{$\mathbf{D}<0$} & $\$, \$, \$, c ; N, N, N$ & 43 \\
\hline & & \multicolumn{3}{|c|}{$\mathbf{D}>0$} & $\$, \$ ; N, N, N$ & 44 \\
\hline & & \multirow{2}{*}{\multicolumn{2}{|c|}{$\mathbf{D}=0$}} & $\mathbf{T} \neq 0$ & $\$, \$, \widehat{c p}_{(2)} ; N, N, N$ & 45 \\
\hline & & & & $\mathbf{T}=0$ & $\$, \widehat{s}_{(3)} ; N, N, N$ & 46 \\
\hline & & & $\mathbf{D}<0$ & & $\$, \$, c, c ; N$ & 47 \\
\hline$\mu_{0}$ & & & $\mathbf{D}>0$ & & $\$, c ; N$ & 48 \\
\hline & & & $\mathbf{D}=0$ & & $\$, c, \widehat{c p}_{(2)} ; N$ & 49 \\
\hline & & & & $\mathbf{D}<0$ & $\$, \$, c ; N, \widehat{\left(\begin{array}{l}1 \\
2\end{array}\right)} P E P-H$ & 50 \\
\hline & & & & $\mathbf{D}>0$ & $\$ ; N, \widehat{\left(\begin{array}{l}1 \\
2\end{array}\right)} P E P-H$ & 51 \\
\hline$\mu_{0}$ & & & & $\mathbf{D}=0$ & $\$, \widehat{c p}_{(2)} ; N, \widehat{\left(\begin{array}{l}1 \\
2\end{array}\right)} P E P-H$ & 52 \\
\hline & & & $\mu_{2} \neq 0$ & $\widetilde{K} \neq 0$ & $\$, \$ ; N,\left(\begin{array}{l}2 \\
2\end{array}\right) P E P-P E P$ & 53 \\
\hline & & $\mu_{1}=0$ & & $\widetilde{K}=0$ & $\$, c ; \widehat{\left(\begin{array}{l}2 \\
3\end{array}\right)} N$ & 54 \\
\hline & & & $\mu_{2}$ & & $\$ ;\left(\begin{array}{l}3 \\
3\end{array}\right) P E P E P-P$ & 55 \\
\hline
\end{tabular}

TABLE 2. Configurations of the finite singularities for Quadratic systems having an integrable saddle: the case $\mathcal{T}_{3}=\mathcal{T}_{2}=0$ 


\begin{tabular}{|c|c|c|c|c|c|}
\hline $\begin{array}{l}\text { Con- } \\
\text { figu- } \\
\text { ration }\end{array}$ & $\begin{array}{l}\text { Phase } \\
\text { portrait }\end{array}$ & \begin{tabular}{|c|} 
Con- \\
figu- \\
ration \\
\end{tabular} & $\begin{array}{l}\text { Phase } \\
\text { portrait }\end{array}$ & \begin{tabular}{|c|} 
Con- \\
figu- \\
ration \\
\end{tabular} & $\begin{array}{l}\text { Phase } \\
\text { portrait }\end{array}$ \\
\hline 1 & 28 & 21 & $\mathrm{Vul}_{2}$ & 41 & $\cong \mathrm{Ham}_{19}$ \\
\hline 2 & 29 & 22 & $V u l_{19}$ & \multirow{2}{*}{42} & 47 if $B_{3} \neq 0$ \\
\hline 3 & 30 & 23 & $\cong \mathrm{Ham}_{25}$ & & 46 if $B_{3}=0$ \\
\hline 4 & 31 & \multirow{3}{*}{24} & $V u l_{18}$ if $B_{3} B_{5}<0$ & \multirow{4}{*}{43} & $V u l_{11}$ if $B_{1} \neq 0$ \\
\hline 5 & 32 & & $V u l_{16}$ if $B_{3} B_{5}>0$ & & $V u l_{9}$ if $B_{1}=0, B_{3} B_{4}<0$ \\
\hline 6 & 30 & & $V u l_{17}$ if $B_{3}=0$ & & $V u l_{8}$ if $B_{1}=0, B_{3} B_{4}>0$ \\
\hline 7 & 31 & 25 & $\cong \mathrm{Ham}_{25}$ & & $V u l_{10}$ if $B_{1}=B_{3}=0$ \\
\hline 8 & 33 & 26 & 35 & \multirow[t]{2}{*}{44} & $\operatorname{Ham}_{25}$ if $\left\{\begin{array}{l}B_{1} \neq 0, \text { or } \\
B_{1}=0, B_{3} B_{4}>0\end{array}\right.$ \\
\hline 9 & $V u l_{25}$ & 27 & 36 & & $\mathrm{Ham}_{26}$ if $B_{1}=0, B_{3} B_{4}<0$ \\
\hline \multirow{3}{*}{10} & $V u l_{9}$ if $B_{3} B_{5}<0$ & 28 & 35 & \multirow{2}{*}{45} & $\mathrm{Ham}_{27}$ if $B_{1} \neq 0$ \\
\hline & $V u l_{8}$ if $B_{3} B_{5}>0$ & 29 & 37 & & $\mathrm{Ham}_{24}$ if $B_{1}=0$ \\
\hline & $V u l_{10}$ if $B_{3}=0$ & 30 & 38 & 46 & $\mathrm{Ham}_{25}$ \\
\hline \multirow{3}{*}{11} & $V u l_{28}$ if $B_{3} B_{5}<0$ & 31 & 39 & \multirow{2}{*}{47} & $V_{u l}$ if $B_{1} \neq 0$ \\
\hline & $V u l_{26}$ if $B_{3} B_{5}>0$ & 32 & 40 & & $V u l_{3}$ if $B_{1}=0$ \\
\hline & $V u l_{27}$ if $B_{3}=0$ & 33 & $\cong \operatorname{Ham}_{19}$ & 48 & $\mathrm{Vul}_{2}$ \\
\hline 12 & $V u l_{25}$ & \multirow{2}{*}{34} & $\cong \operatorname{Ham}_{20}$ if $B_{3} \neq 0$ & 49 & $\overline{V u l_{7}}$ \\
\hline 13 & $\cong \mathrm{Ham}_{25}$ & & $\cong \mathrm{Ham}_{21}$ if $\mathrm{B}_{3}=0$ & \multirow{2}{*}{50} & $V u l_{6}$ if $B_{1} \neq 0$ \\
\hline 14 & 34 & \multirow{2}{*}{35} & 41 if $B_{3} \neq 0$ & & $V u l_{5}$ if $B_{1}=0$ \\
\hline 15 & 29 & & 42 if $B_{3}=0$ & 51 & $H_{a m} 19$ \\
\hline 16 & 34 & 36 & 43 & 52 & $\mathrm{Ham}_{22}$ \\
\hline 17 & $V u l_{2}$ & 37 & 44 & \multirow{2}{*}{53} & $H_{a m} 20$ if $B_{3} \neq 0$ \\
\hline 18 & $V u l_{19}$ & 38 & 45 & & $\mathrm{Ham}_{21}$ if $B_{3}=0$ \\
\hline 19 & $V u l_{2}$ & 39 & 46 & 54 & $V u l_{2}$ \\
\hline 20 & $V u l_{2}$ & 40 & $\cong \operatorname{Ham}_{19}$ & 55 & $\mathrm{Ham}_{18}$ \\
\hline
\end{tabular}

TABLE 3. The conditions for the realization of each one of the phase portraits of Quadratic systems having an integrable saddle

\section{Some PRELIMINARY RESUltS}

Consider real quadratic systems of the form:

$$
\begin{aligned}
& \frac{d x}{d t}=p_{0}+p_{1}(x, y)+p_{2}(x, y) \equiv P(x, y), \\
& \frac{d y}{d t}=q_{0}+q_{1}(x, y)+q_{2}(x, y) \equiv Q(x, y)
\end{aligned}
$$

with homogeneous polynomials $p_{i}$ and $q_{i}(i=0,1,2)$ of degree $i$ in $x, y$ :

$$
\begin{array}{r}
p_{0}=a_{00}, \quad p_{1}(x, y)=a_{10} x+a_{01} y, \quad p_{2}(x, y)+a_{20} x^{2}+2 a_{11} x y+a_{02} y^{2}, \\
q_{0}=b_{00}, \quad q_{1}(x, y)=b_{10} x+b_{01} y, \quad q_{2}(x, y)+b_{20} x^{2}+2 b_{11} x y+b_{02} y^{2} .
\end{array}
$$

Let $\tilde{a}=\left(a_{00}, a_{10}, a_{01}, a_{20}, a_{11}, a_{02}, b_{00}, b_{10}, b_{01}, b_{20}, b_{11}, b_{02}\right)$ be the 12 -tuple of the coefficients of systems $(7)$ and denote $\mathbb{R}[\tilde{a}, x, y]=\mathbb{R}\left[a_{00}, \ldots, b_{02}, x, y\right]$.

Definitions and notations (see [13] for a more detailed description).

It is well-known that singular points of polynomial differential systems can be split into four main groups according to the linear part of the system at them. If we denote by $\Delta$ and by $\rho$ the determinant and the trace of the linear part $J$ of the system at a singular point we have 

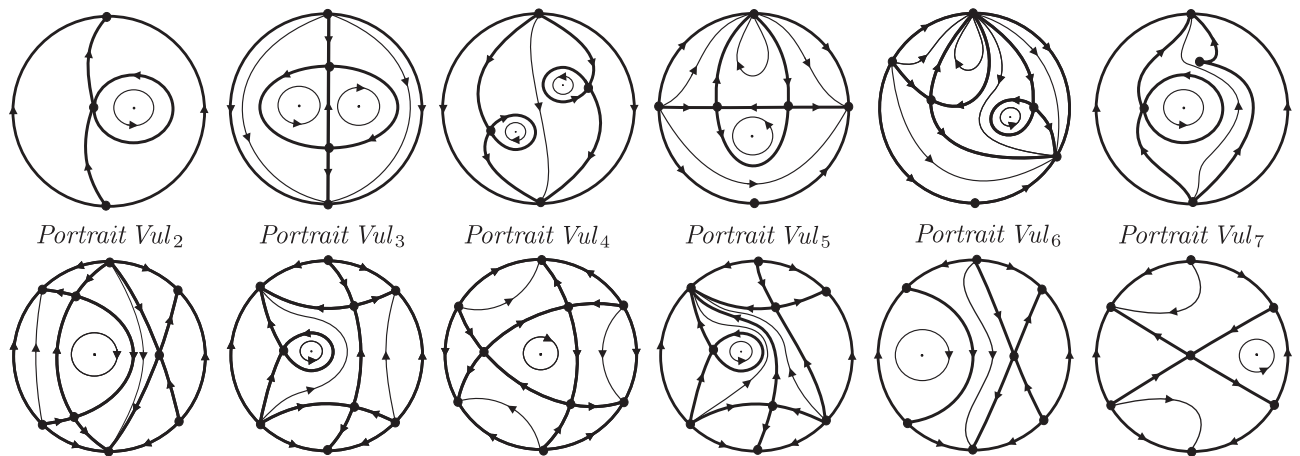

Portrait $\mathrm{Vul}_{3}$

Portrait $\mathrm{Vul}_{4}$

Portrait $\mathrm{Vul}_{5}$

Portrait $\mathrm{Vul}_{6}$

Portrait $\mathrm{Vul}_{7}$
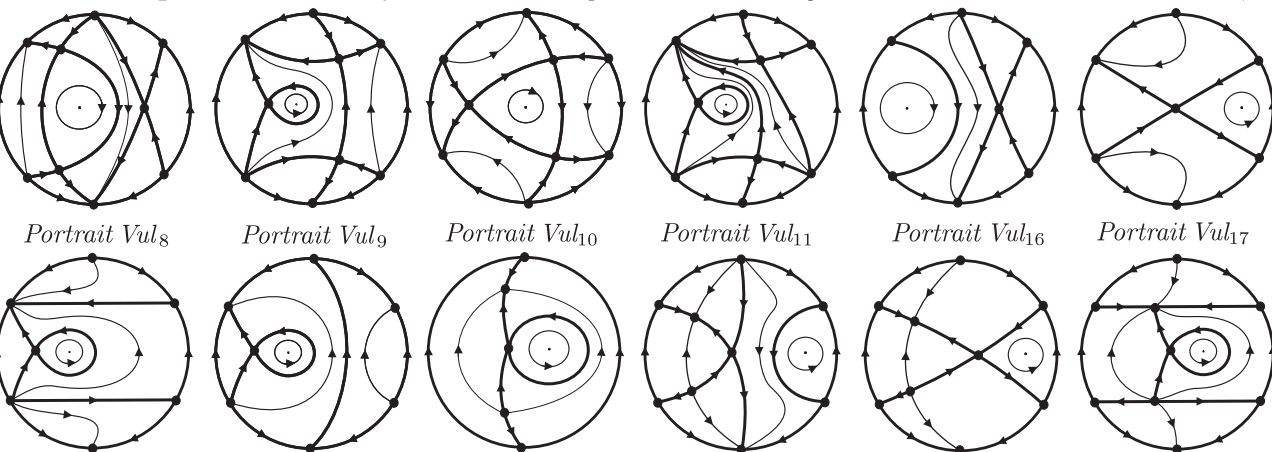

Portrait Vul $_{9}$

Portrait $\mathrm{Vul}_{10}$

Portrait $V u l_{11}$

Portrait $V_{u l} l_{16}$

Portrait $\mathrm{Vul}_{17}$
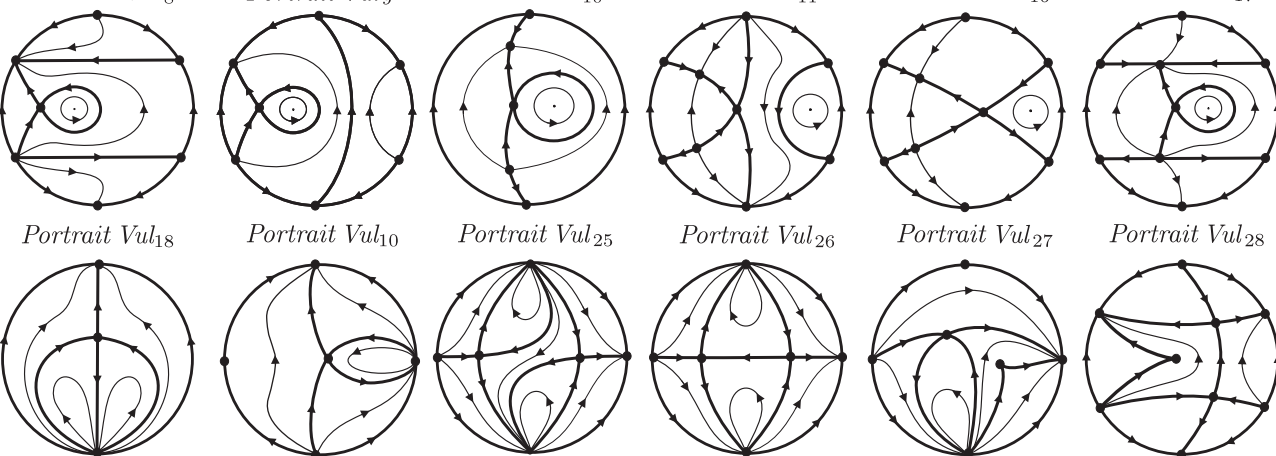

Portrait $V_{u l}$

Portrait $V u l_{26}$

Portrait $V u l_{27}$
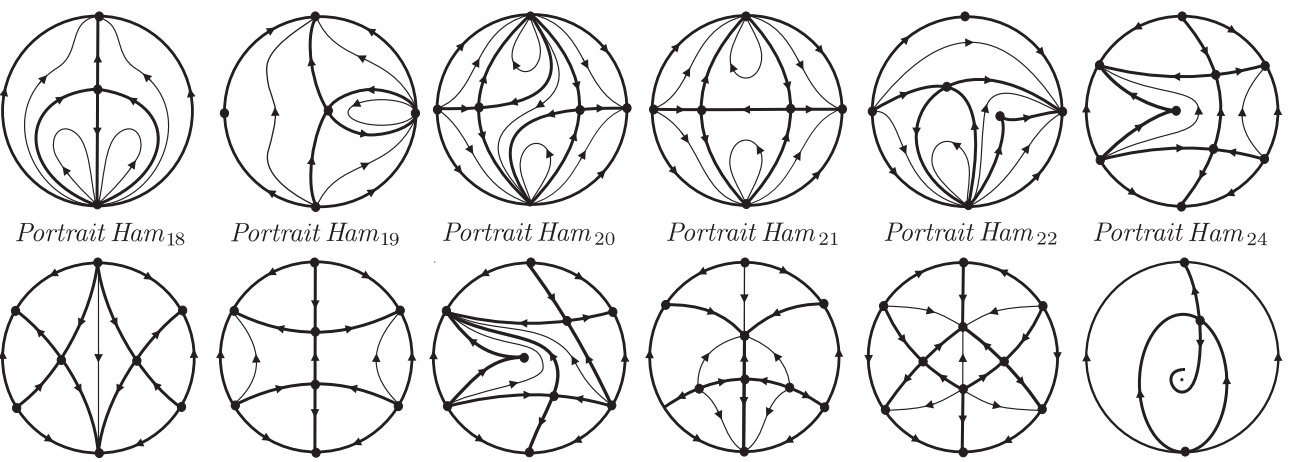

Portrait Ham 20

Portrait Ham 21

Portrait Ham 22

Portrait $\mathrm{Ham}_{24}$

Portrait Ham $_{25}$
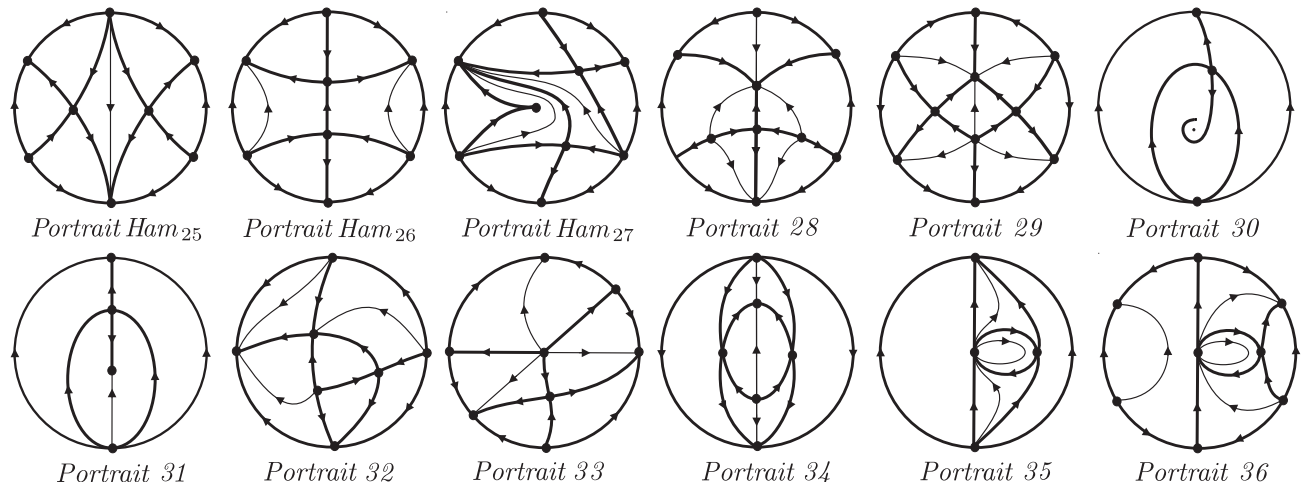

Portrait Ham $_{27}$
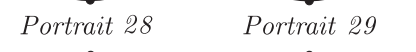

Portrait 30
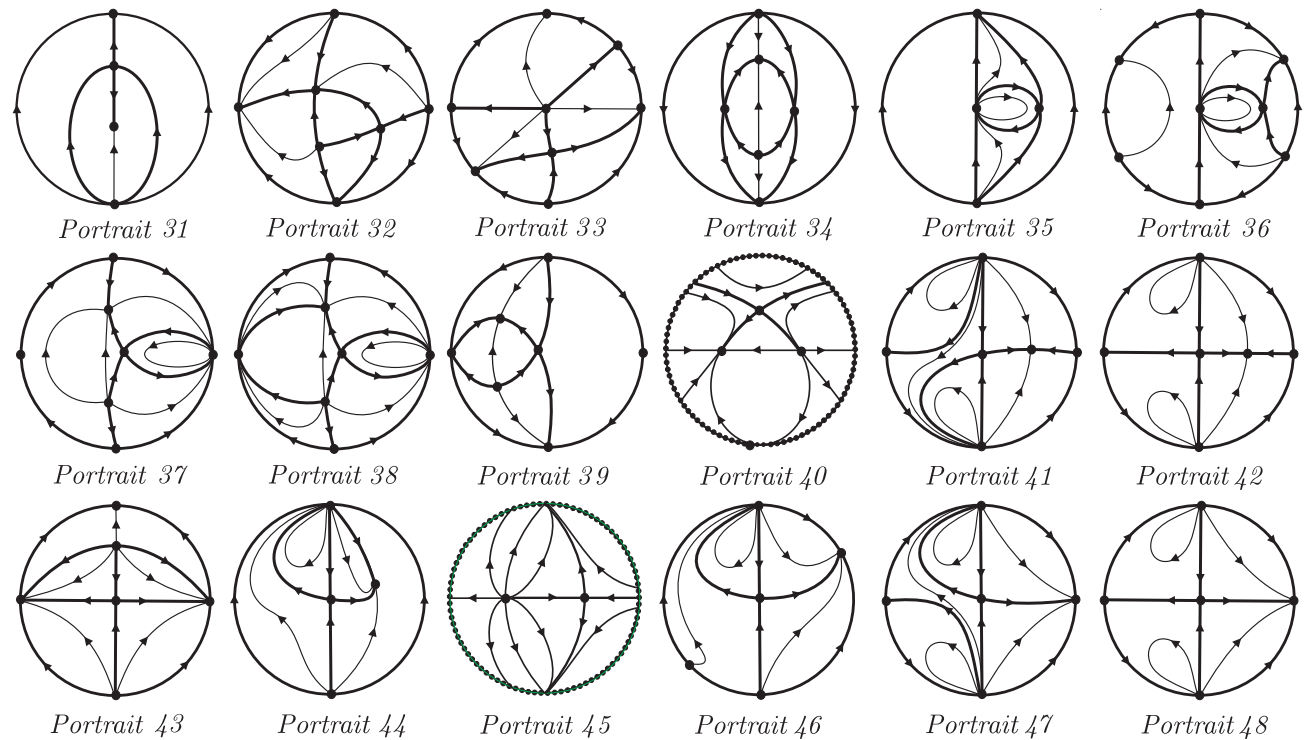

FiguRE 1. Phase portraits of Quadratic systems having an integrable saddle

that a point is elementary (respectively semi-elementary, nilpotent and degenerate) if $\Delta \neq 0$, (respectively $\Delta=0 \neq \rho, \Delta=0=\rho$, but $J \not \equiv 0$, and $J \equiv 0$ ). 
Moreover, an elementary point can be a saddle " $s$ ", a node " $n$ ", a focus " $f$ " or a center " $c$ "; a semi-elementary point can be a saddle " $\bar{s}_{(\#)}$ ", a node " $\bar{n}_{(\#)}$ " or a saddle-node " $\overline{s n}_{(\#)}$ ", a nilpotent point can be a saddle " $\hat{s}_{(\#)}$ ", a node " $\hat{n}_{(\#)}$ ", a saddle-node " $\widehat{s n}_{(\#)}$ ", an elliptic-saddle " $\widehat{e s}_{(\#)}$ ", a cusp " $\widehat{c p}_{(\#)}$ ", a focus " $\hat{f}_{(\#)}$ " or a center " $\hat{c}_{(\#)}$ "; and a degenerated point is either a focus, a center or its neighborhood is formed by a finite number (for polynomial systems) of elliptic, parabolic and/or hyperbolic sectors, and it can be described as " $s_{1} s_{2} \ldots s_{n(\#)}$ " where $s_{i} \in\{e, p, h\}$ and using clock-wise sense. The subindex "\#" stands in all the cases for the multiplicity of the point, that is, the maximum number of singular points that can bifurcate from it under perturbation. Not all of them can appear in quadratic systems, and some of them as the $\widehat{e s}_{(\#)}$ and the $\widehat{c p}_{(\#)}$ can only appear with the multiplicities 3 and 2 respectively. We have named all nilpotent points even they do not appear in quadratic systems for coherence with other nilpotent points but we have not named the degenerated centers and focus since they neither appear and a new notation is needed which we let for future studies of higher degree systems.

When considering weak elementary singularities, it is known that we can split saddles and focus according to their degree of weakness and that weakness for quadratic systems is limited to three degrees plus an integrable level, either for saddles or foci. So we will distinguish them as " $s(\#)$ " and " $f(\#)$ " for $\# \in\{1,2,3\}$ the respective weak saddles and weak focus of first, second and third order leaving the $s$ and $f$ for the non weak ones. The next degree of weakness for the focus of quadratic systems correspond to centers and the next degree of weakness for the saddles are the integrable saddles which we will denote by "\$".

It is also well known that planar polynomial differential systems can be extended to a sphere and projected onto a disk providing in this way a compactified view of all the flow including the asymptotic behavior close to infinity. This can be done using different compactifying techniques of which we chose the Poincaré compactification. From the infinite local charts point of view, the system has one degree more that in the finite plane, and the equator of the sphere corresponds to the infinity which is always invariant and may contain also infinite singular points. We will denote the infinite singular points in the same way as we denote finite ones (using capital letter for the first and lower case for the latter) except that we will distinguish the multiplicity of the infinite points with the notation $\left(\begin{array}{l}f \\ i\end{array}\right)$ where $f$ stands for the number of finite points that can bifurcate from it and $i$ for the infinite. In case they are simple we will avoid the use of the term $\left(\begin{array}{l}0 \\ 1\end{array}\right)$. We add a dash to separate the sectors of nilpotent and degenerate singular points which are located on one side of the infinity, from those of the opposite side. For example, the notation $\left(\begin{array}{l}3 \\ 2\end{array}\right) P E P-P H$ corresponds to a singular point formed by the collision of three finite points and two infinite, having a sequence of parabolic, elliptic and parabolic sectors on one side and parabolic and hyperbolic on the other. We write a semicolon to split the finite and infinite singular points.

The infinity may be formed by an infinite set of singular points. In that case, in the expression of the differential system in the infinite local chart, there is a common factor which may be removed and a lower degree system remains which must be studied. Possibly the system has not the line of infinity invariant any more, but may still contain singular points. In [29] all quadratic systems with the line of infinity filled with singular points were studied and it was determined that the neighborhood of infinity may be of 6 different classes. Two of them will appear in this paper and we will denote Figure 41 of $[29]$ as $(\infty, \emptyset)$ and Figure 44 as $(\infty, N)$ according to the type of the singular point which appears at infinity after the removal of the singular line (in the first case there is no singular point after). The other classes can be denoted in a similar way.

Among all the orbits which form the flow of a differential system, the most interesting ones are the so called separatrices. Briefly speaking these are the orbits which border the hyperbolic sectors, plus the limit cycles and the singular points. Removing all the separatrices, the disk splits in a set of canonical regions and adding one orbit to each one of them, the global phase portrait is completed (see for more details [13]).

In this paper we are going to draw all separatrices, plus the line of infinity with a wide line and we will add some orbits with a thinner line to make it perfectly understandable. In order 
not to overload the picture we will skip the extra orbit in some regions where the attractor or repeller sets in the boundary are easy to detect. More concretely we will skip extra orbit except in the next situations, when we will draw:

1) a closed orbit around a center;

2) an orbit in each elliptic sector;

3) an orbit arriving to (or departing from) any node or focus which is not source/sink of any finite separatrix;

4) an orbit on each parabolic sector of a singular point not topologically equivalent to an elementary point;

$5)$ one orbit inside any canonical region which border includes 4 or more singular points;

6) some extra orbits when the infinite is filled with singular points;

7) the needed orbits to complete invariant straight lines.

We will draw a large dot for the singular points except for the focus and centers for which a small dot is enough. If there is a set of an infinite number of singular points (in the finite or infinite part) we will denote it with a continuous set of dots.

2.1. Weak singularities of the family of quadratic systems. In [32] we have the following result.

Theorem 2. Consider a non-degenerate quadratic system (7).

(a) If $\mathcal{T}_{4} \neq 0$ then this system has no weak singularity.

(b) If $\mathcal{T}_{4}=0$ and $\mathcal{T}_{3} \neq 0$ then the system has exactly one weak singularity. Moreover this singularity is either a weak focus (respectively a weak saddle) of the indicated order below, or a center (respectively an integrable saddle) if and only if $\mathcal{T}_{3} \mathcal{F}<0$ (respectively $\mathcal{T}_{3} \mathcal{F}>0$ ) and the following corresponding condition holds:

$\left(b_{1}\right) \quad f^{(1)}$ (respectively $\left.s^{(1)}\right) \Leftrightarrow \mathcal{F}_{1} \neq 0$;

$\left(b_{2}\right) \quad f^{(2)}$ (respectively $\left.s^{(2)}\right) \Leftrightarrow \mathcal{F}_{1}=0, \mathcal{F}_{2} \neq 0$

$\left(b_{3}\right) \quad f^{(3)}$ (respectively $\left.s^{(3)}\right) \Leftrightarrow \mathcal{F}_{1}=\mathcal{F}_{2}=0, \mathcal{F}_{3} \mathcal{F}_{4} \neq 0$;

$\left(b_{4}\right) \quad c \quad($ respectively $\$) \quad \Leftrightarrow \quad \mathcal{F}_{1}=\mathcal{F}_{2}=\mathcal{F}_{3} \mathcal{F}_{4}=0$.

(c) If $\mathcal{T}_{4}=\mathcal{T}_{3}=0$ and $\mathcal{T}_{2} \neq 0$ then the system could possess two and only two weak singularities and none of them is of order 2 or 3 . Moreover this system possesses two weak singularities, which are of the types indicated below, if and only if $\mathcal{F}=0$ and one of the following conditions holds:

$\left(c_{1}\right) \quad s^{(1)}, s^{(1)} \Leftrightarrow \mathcal{F}_{1} \neq 0, \mathcal{T}_{2}<0, \mathcal{B} \leq 0, \mathcal{H}>0$

$\left(c_{2}\right) \quad s^{(1)}, f^{(1)} \Leftrightarrow \mathcal{F}_{1} \neq 0, \mathcal{T}_{2}>0, \mathcal{B}<0$;

$\left(c_{3}\right) \quad f^{(1)}, f^{(1)} \Leftrightarrow \mathcal{F}_{1} \neq 0, \mathcal{T}_{2}<0, \mathcal{B}<0, \mathcal{H}<0$

$\left(c_{4}\right) \quad \$, \quad \$ \quad \Leftrightarrow \quad \mathcal{F}_{1}=0, \mathcal{T}_{2}<0, \mathcal{B}<0, \mathcal{H}>0$

$\left(c_{5}\right) \quad \$, \quad c \quad \Leftrightarrow \quad \mathcal{F}_{1}=0, \mathcal{T}_{2}>0, \mathcal{B}<0$;

$\left(c_{6}\right) \quad c, \quad c \quad \Leftrightarrow \quad \mathcal{F}_{1}=0, \mathcal{T}_{2}<0, \mathcal{B}<0, \mathcal{H}<0$.

(d) If $\mathcal{T}_{4}=\mathcal{T}_{3}=\mathcal{T}_{2}=0$ and $\mathcal{T}_{1} \neq 0$ then the system could possess one and only one weak singularity (which is of order 1). Moreover this system has one weak singularity of the type indicated below if and only if $\mathcal{F}=0$ and one of the following conditions holds:

$\left(d_{1}\right) \quad s^{(1)} \Leftrightarrow \mathcal{F}_{1} \neq 0, \mathcal{B}<0, \mathcal{H}>0$

$\left(d_{2}\right) \quad f^{(1)} \Leftrightarrow \mathcal{F}_{1} \neq 0, \mathcal{B}<0, \mathcal{H}<0$.

(e) If $\mathcal{T}_{4}=\mathcal{T}_{3}=\mathcal{T}_{2}=\mathcal{T}_{1}=0$ and $\sigma(a, x, y) \neq 0$ then the system could possess one and only only one weak singularity. Moreover this system has one weak singularity, which 
is of the type indicated below, if and only if one of the following conditions holds:

$$
\begin{aligned}
& \left(\begin{array}{ll}
\left(e_{1}\right) s^{(1)} \\
\left(e_{2}\right) f^{(1)} \Leftrightarrow \quad \mathcal{F}_{1} \neq 0, \mathcal{H}=\mathcal{B}_{1}=0, \mathcal{B}_{2}>0 ;
\end{array}\right. \\
& \left(e_{3}\right) \quad \$ \quad \Leftrightarrow \begin{cases}{[\alpha]} & \mathcal{F}_{1}=0, \mathcal{H}=0, \mathcal{B}<0, \mathcal{H}>0, \text { or } \\
{[\beta]} & \mathcal{F}_{1}=0, \mathcal{H}=\mathcal{B}_{1}=0, \mathcal{B}_{2}>0, \text { or } \\
{[\gamma]} & \mathcal{F}_{1}=0, \mathcal{H}=\mathcal{B}=\mathcal{B}_{1}=\mathcal{B}_{2}=\mathcal{B}_{3}=\mu_{0}=0, K\left(\mu_{2}^{2}+\mu_{3}^{2}\right) \neq 0, \text { or } \\
{[\delta]} & \mathcal{F}_{1}=0, \mathcal{H}=\mathcal{B}=\mathcal{B}_{1}=\mathcal{B}_{2}=\mathcal{B}_{3}=K=0, \mu_{2} \mathcal{G} \neq 0, \text { or } \\
{[\varepsilon]} & \mathcal{F}_{1}=0, \mathcal{H}=\mathcal{B}=\mathcal{B}_{1}=\mathcal{B}_{2}=\mathcal{B}_{3}=\mathcal{B}_{4}=K=\mu_{2}=0, \mu_{3} \neq 0 ;\end{cases} \\
& \left(e_{4}\right) \quad c \quad \Leftrightarrow \begin{cases}{[\alpha]} & \mathcal{F}_{1}=0, \mathcal{F}=0, \mathcal{B}<0, \mathcal{H}<0, \text { or } \\
{[\beta]} & \mathcal{F}_{1}=0, \mathcal{H}=\mathcal{B}_{1}=0, \mathcal{B}_{2}<0 .\end{cases}
\end{aligned}
$$

(f) If $\sigma(a, x, y)=0$ then the system is Hamiltonian and it possesses $i$ (with $1 \leq i \leq 4$ ) weak singular points of the types indicated below if and only if one of the following conditions holds:

$$
\begin{aligned}
& \left(f_{1}\right) \quad \$, \$, \$, c \quad \Leftrightarrow \quad \mu_{0}<0, \mathbf{D}<0, \mathbf{R}>0, \mathbf{S}>0 ; \\
& \left(f_{2}\right) \quad \$, \$, c, c \quad \Leftrightarrow \quad \mu_{0}>0, \mathbf{D}<0, \mathbf{R}>0, \mathbf{S}>0 ; \\
& \left(f_{3}\right) \quad \$, \$, c \quad \Leftrightarrow \quad \mu_{0}=0, \mathbf{D}<0, \mathbf{R} \neq 0 \text {; } \\
& \left(f_{4}\right) \quad \$, \$ \quad \Leftrightarrow \quad \begin{cases}{[\alpha]} & \mu_{0}<0, \mathbf{D}>0, \text { or } \\
{[\beta]} & \mu_{0}<0, \mathbf{D}=0, \mathbf{T}<0, \text { or } \\
{[\gamma]} & \mu_{0}=\mathbf{R}=0, \mathbf{P} \neq 0, \mathbf{U}>0, K \neq 0 ;\end{cases} \\
& \left(f_{5}\right) \quad \$, c \quad \Leftrightarrow \quad \begin{cases}{[\alpha]} & \mu_{0}>0, \mathbf{D}>0, \text { or } \\
{[\beta]} & \mu_{0}>0, \mathbf{D}=0, \mathbf{T}<0, \text { or } \\
{[\gamma]} & \mu_{0}=\mathbf{R}=0, \mathbf{P} \neq 0, \mathbf{U}>0, K=0 ;\end{cases} \\
& \left(f_{6}\right) \quad \$ \quad \Leftrightarrow \begin{cases}{[\alpha]} & \mu_{0}<0, \mathbf{D}=\mathbf{T}=\mathbf{P}=0, \mathbf{R} \neq 0, \text { or } \\
{[\beta]} & \mu_{0}=0, \mathbf{D}>0, \mathbf{R} \neq 0, \text { or } \\
{[\gamma]} & \mu_{0}=0, \mathbf{D}=0, \mathbf{P R} \neq 0, \text { or } \\
{[\delta]} & \mu_{0}=\mathbf{R}=\mathbf{P}=0, \mathbf{U} \neq 0 ;\end{cases} \\
& \left(f_{7}\right) \quad c \quad \Leftrightarrow \quad \mu_{0}>0, \mathbf{D}=\mathbf{T}=\mathbf{P}=0, \mathbf{R} \neq 0 .
\end{aligned}
$$

Here the invariant polynomials are constructed as follows.

We single out the following five polynomials, basic ingredients in constructing invariant polynomials for systems (7):

$$
\begin{aligned}
& C_{i}(\tilde{a}, x, y)=y p_{i}(x, y)-x q_{i}(x, y), \quad(i=0,1,2) \\
& D_{i}(\tilde{a}, x, y)=\frac{\partial p_{i}}{\partial x}+\frac{\partial q_{i}}{\partial y},(i=1,2) .
\end{aligned}
$$

Using the so-called transvectant of index $k$ (see [18]) of two polynomials $f, g \in \mathbb{R}[\tilde{a}, x, y]$

$$
(f, g)^{(k)}=\sum_{h=0}^{k}(-1)^{h}\left(\begin{array}{l}
k \\
h
\end{array}\right) \frac{\partial^{k} f}{\partial x^{k-h} \partial y^{h}} \frac{\partial^{k} g}{\partial x^{h} \partial y^{k-h}},
$$

we construct the following $G L$-comitants of the second degree with respect to the coefficients of the initial system

$$
\begin{array}{lll}
T_{1}=\left(C_{0}, C_{1}\right)^{(1)}, & T_{2}=\left(C_{0}, C_{2}\right)^{(1)}, & T_{3}=\left(C_{0}, D_{2}\right)^{(1)}, \\
T_{4}=\left(C_{1}, C_{1}\right)^{(2)}, & T_{5}=\left(C_{1}, C_{2}\right)^{(1)}, & T_{6}=\left(C_{1}, C_{2}\right)^{(2)}, \\
T_{7}=\left(C_{1}, D_{2}\right)^{(1)}, & T_{8}=\left(C_{2}, C_{2}\right)^{(2)}, & T_{9}=\left(C_{2}, D_{2}\right)^{(1)} .
\end{array}
$$

Consider now the differential operator $\mathcal{L}=x \cdot \mathbf{L}_{2}-y \cdot \mathbf{L}_{1}$ (see [7]) acting on $\mathbb{R}[\tilde{a}, x, y]$, where

$$
\begin{aligned}
& \mathbf{L}_{1}=2 a_{00} \frac{\partial}{\partial a_{10}}+a_{10} \frac{\partial}{\partial a_{20}}+\frac{1}{2} a_{01} \frac{\partial}{\partial a_{11}}+2 b_{00} \frac{\partial}{\partial b_{10}}+b_{10} \frac{\partial}{\partial b_{20}}+\frac{1}{2} b_{01} \frac{\partial}{\partial b_{11}} \\
& \mathbf{L}_{2}=2 a_{00} \frac{\partial}{\partial a_{01}}+a_{01} \frac{\partial}{\partial a_{02}}+\frac{1}{2} a_{10} \frac{\partial}{\partial a_{11}}+2 b_{00} \frac{\partial}{\partial b_{01}}+b_{01} \frac{\partial}{\partial b_{02}}+\frac{1}{2} b_{10} \frac{\partial}{\partial b_{11}} .
\end{aligned}
$$


Using this operator we construct the following important set of invariant polynomials:

$$
\begin{aligned}
& \mu_{0}(\tilde{a})=\operatorname{Res}_{x}\left(p_{2}(x, y), q_{2}(x, y)\right) / y^{4}, \\
& \mu_{i}(\tilde{a}, x, y)=\frac{1}{i !} \mathcal{L}^{(i)}\left(\mu_{0}\right), i=1, . ., 4,
\end{aligned}
$$

where $\mathcal{L}^{(i)}\left(\mu_{0}\right)=\mathcal{L}\left(\mathcal{L}^{(i-1)}\left(\mu_{0}\right)\right)$.

We denote

$$
\sigma(\tilde{a}, x, y)=\frac{\partial P}{\partial x}+\frac{\partial Q}{\partial y}=\sigma_{0}(\tilde{a})+\sigma_{1}(\tilde{a}, x, y)\left(\equiv D_{1}(\tilde{a})+D_{2}(\tilde{a}, x, y)\right)
$$

and observe that the polynomial $\sigma(\tilde{a}, x, y)$ is an affine comitant of systems (7). It is known, that if $\left(x_{i}, y_{i}\right)$ is a singular point of a system (7) then for the trace of its respective linear matrix we have $\rho_{i}=\sigma\left(x_{i}, y_{i}\right)$.

Applying the differential operators $\mathcal{L}$ and $(*, *)^{(k)}$ (i.e. transvectant of index $k$ ) we shall define the following polynomial function which governs the values of the traces for finite singularities of systems (7).

Definition 1 ([32]). We call the trace function $\mathfrak{T}(w)$ the function defined as follows:

$$
\mathfrak{T}(w)=\sum_{i=0}^{4} \frac{1}{(i !)^{2}}\left(\sigma_{1}^{i}, \frac{1}{i !} \mathcal{L}^{(i)}\left(\mu_{0}\right)\right)^{(i)} w^{4-i}=\sum_{i=0}^{4} \mathcal{G}_{i} w^{4-i}
$$

where the coefficients $\mathcal{G}_{i}(\tilde{a})=\frac{1}{(i !)^{2}}\left(\sigma_{1}^{i}, \mu_{i}\right)^{(i)}, i=0,1,2,3,4\left(\mathcal{G}_{0}(\tilde{a}) \equiv \mu_{0}(\tilde{a})\right)$ are $G L-$ invariants.

Using the function $\mathfrak{T}(w)$ we could construct the following four affine invariants $\mathcal{T}_{4}, \mathcal{T}_{3}, \mathcal{T}_{2}$, $\mathcal{T}_{1}$ :

$$
\mathcal{T}_{4-i}(\tilde{a})=\left.\frac{1}{i !} \frac{d^{i} \mathfrak{T}}{d w^{i}}\right|_{w=\sigma_{0}}, i=0,1,2,3 \quad\left(\mathcal{T}_{4} \equiv \mathfrak{T}\left(\sigma_{0}\right)\right)
$$

In order to be able to calculate the values of the needed invariant polynomials directly for every canonical system we shall define here a family of $T$-comitants (see [25] for detailed 
definitions) expressed through $C_{i}(i=0,1,2)$ and $D_{j}(j=1,2)$ :

$$
\begin{aligned}
\widetilde{A}= & \left(C_{1}, T_{8}-2 T_{9}+D_{2}^{2}\right)^{(2)} / 144 \\
\widetilde{D}= & {\left[2 C_{0}\left(T_{8}-8 T_{9}-2 D_{2}^{2}\right)+C_{1}\left(6 T_{7}-T_{6}-\left(C_{1}, T_{5}\right)^{(1)}+6 D_{1}\left(C_{1} D_{2}-T_{5}\right)-9 D_{1}^{2} C_{2}\right] / 36,\right.} \\
\widetilde{E}= & {\left[D_{1}\left(2 T_{9}-T_{8}\right)-3\left(C_{1}, T_{9}\right)^{(1)}-D_{2}\left(3 T_{7}+D_{1} D_{2}\right)\right] / 72, } \\
\widetilde{F}= & {\left[6 D_{1}^{2}\left(D_{2}^{2}-4 T_{9}\right)+4 D_{1} D_{2}\left(T_{6}+6 T_{7}\right)+48 C_{0}\left(D_{2}, T_{9}\right)^{(1)}-9 D_{2}^{2} T_{4}+288 D_{1} \widetilde{E}\right.} \\
& \left.-24\left(C_{2}, \widetilde{D}\right)^{(2)}+120\left(D_{2}, \widetilde{D}\right)^{(1)}-36 C_{1}\left(D_{2}, T_{7}\right)^{(1)}+8 D_{1}\left(D_{2}, T_{5}\right)^{(1)}\right] / 144, \\
\widetilde{B}= & 16 D_{1}\left(D_{2}, T_{8}\right)^{(1)}\left(3 C_{1} D_{1}-2 C_{0} D_{2}+4 T_{2}\right)+32 C_{0}\left(D_{2}, T_{9}\right)^{(1)}\left(3 D_{1} D_{2}-5 T_{6}+9 T_{7}\right) \\
& +2\left(D_{2}, T_{9}\right)^{(1)}\left(27 C_{1} T_{4}-18 C_{1} D_{1}^{2}-32 D_{1} T_{2}+32\left(C_{0}, T_{5}\right)^{(1)}\right) \\
& +6\left(D_{2}, T_{7}\right)^{(1)}\left[8 C_{0}\left(T_{8}-12 T_{9}\right)-12 C_{1}\left(D_{1} D_{2}+T_{7}\right)+D_{1}\left(26 C_{2} D_{1}+32 T_{5}\right)+C_{2}\left(9 T_{4}+96 T_{3}\right)\right] \\
& +6\left(D_{2}, T_{6}\right)^{(1)}\left[32 C_{0} T_{9}-C_{1}\left(12 T_{7}+52 D_{1} D_{2}\right)-32 C_{2} D_{1}^{2}\right]+48 D_{2}\left(D_{2}, T_{1}\right)^{(1)}\left(2 D_{2}^{2}-T_{8}\right) \\
& -32 D_{1} T_{8}\left(D_{2}, T_{2}\right)^{(1)}+9 D_{2}^{2} T_{4}\left(T_{6}-2 T_{7}\right)-16 D_{1}\left(C_{2}, T_{8}\right)^{(1)}\left(D_{1}^{2}+4 T_{3}\right) \\
& +12 D_{1}\left(C_{1}, T_{8}\right)^{(2)}\left(C_{1} D_{2}-2 C_{2} D_{1}\right)+6 D_{1} D_{2} T_{4}\left(T_{8}-7 D_{2}^{2}-42 T_{9}\right) \\
& +12 D_{1}\left(C_{1}, T_{8}\right)^{(1)}\left(T_{7}+2 D_{1} D_{2}\right)+96 D_{2}^{2}\left[D_{1}\left(C_{1}, T_{6}\right)^{(1)}+D_{2}\left(C_{0}, T_{6}\right)^{(1)}\right] \\
& -16 D_{1} D_{2} T_{3}\left(2 D_{2}^{2}+3 T_{8}\right)-4 D_{1}^{3} D_{2}\left(D_{2}^{2}+3 T_{8}+6 T_{9}\right)+6 D_{1}^{2} D_{2}^{2}\left(7 T_{6}+2 T_{7}\right) \\
& \left.-252 D_{1} D_{2} T_{4} T_{9}\right\} /\left(2^{8} 3^{3}\right) . \\
\widetilde{K}= & \left(T_{8}+4 T_{9}+4 D_{2}^{2}\right) / 72 \equiv\left(p_{2}(x, y), q_{2}(x, y)\right)^{(1)} / 4, \\
\widetilde{H}= & \left.-T_{8}+8 T_{9}+2 D_{2}^{2}\right) / 72, \\
\widetilde{M}= & T_{8} \\
& +
\end{aligned}
$$

These polynomials in addition to (8) and (9) will serve as bricks in constructing affine algebraic invariants for systems (7). Using these bricks the minimal polynomial basis of affine invariants up to degree 12 , containing 42 elements $A_{1}, \ldots, A_{42}$, was constructed in [9]. The following are 
the elements of this polynomial basis:

$$
\begin{aligned}
& A_{1}=\tilde{A}, \\
& A_{2}=\left(C_{2}, \widetilde{D}\right)^{(3)} / 12 \text {, } \\
& \left.\left.\left.\left.A_{22}=\frac{1}{1152}\left[C_{2}, \widetilde{D}\right)^{(1)}, D_{2}\right)^{(1)}, D_{2}\right)^{(1)}, D_{2}\right)^{(1)} D_{2}\right)^{(1)} \text {, } \\
& \left.\left.A_{3}=\left[C_{2}, D_{2}\right)^{(1)}, D_{2}\right)^{(1)}, D_{2}\right)^{(1)} / 48 \text {, } \\
& \left.A_{23}=[\widetilde{F}, \widetilde{H})^{(1)}, \widetilde{K}\right)^{(2)} / 8 \text {, } \\
& A_{4}=(\widetilde{H}, \widetilde{H})^{(2)} \text {, } \\
& \left.\left.A_{24}=\left[C_{2}, \widetilde{D}\right)^{(2)}, \widetilde{K}\right)^{(1)}, \widetilde{H}\right)^{(2)} / 32 \text {, } \\
& A_{5}=(\widetilde{H}, \widetilde{K})^{(2)} / 2 \text {, } \\
& \left.A_{25}=[\widetilde{D}, \widetilde{D})^{(2)}, \widetilde{E}\right)^{(2)} / 16 \text {, } \\
& A_{6}=(\widetilde{E}, \widetilde{H})^{(2)} / 2 \text {, } \\
& A_{26}=(\widetilde{B}, \widetilde{D})^{(3)} / 36 \text {, } \\
& \left.A_{7}=\left[C_{2}, \widetilde{E}\right)^{(2)}, D_{2}\right)^{(1)} / 8 \text {, } \\
& \left.A_{27}=\left[\widetilde{B}, D_{2}\right)^{(1)}, \widetilde{H}\right)^{(2)} / 24 \text {, } \\
& \left.A_{8}=[\widetilde{D}, \widetilde{H})^{(2)}, D_{2}\right)^{(1)} / 8 \text {, } \\
& \left.\left.A_{28}=\left[C_{2}, \widetilde{K}\right)^{(2)}, \widetilde{D}\right)^{(1)}, \widetilde{E}\right)^{(2)} / 16 \text {, } \\
& \left.\left.A_{9}=\left[\widetilde{D}, D_{2}\right)^{(1)}, D_{2}\right)^{(1)}, D_{2}\right)^{(1)} / 48 \text {, } \\
& \left.A_{29}=[\widetilde{D}, \widetilde{F})^{(1)}, \widetilde{D}\right)^{(3)} / 96 \text {, } \\
& \left.A_{10}=[\widetilde{D}, \widetilde{K})^{(2)}, D_{2}\right)^{(1)} / 8 \text {, } \\
& \left.\left.A_{30}=\left[C_{2}, \widetilde{D}\right)^{(2)}, \widetilde{D}\right)^{(1)}, \widetilde{D}\right)^{(3)} / 288 \text {, } \\
& A_{11}=(\widetilde{F}, \widetilde{K})^{(2)} / 4 \text {, } \\
& \left.\left.A_{31}=[\widetilde{D}, \widetilde{D})^{(2)}, \widetilde{K}\right)^{(1)}, \widetilde{H}\right)^{(2)} / 64 \text {, } \\
& \left.\left.\left.A_{32}=[\widetilde{D}, \widetilde{D})^{(2)}, D_{2}\right)^{(1)}, \widetilde{H}\right)^{(1)}, D_{2}\right)^{(1)} / 64 \text {, } \\
& A_{12}=(\widetilde{F}, \widetilde{H})^{(2)} / 4 \text {, } \\
& \left.\left.\left.A_{33}=\left[\widetilde{D}, D_{2}\right)^{(1)}, \widetilde{F}\right)^{(1)}, D_{2}\right)^{(1)}, D_{2}\right)^{(1)} / 128 \text {, } \\
& \left.\left.A_{13}=\left[C_{2}, \widetilde{H}\right)^{(1)}, \widetilde{H}\right)^{(2)}, D_{2}\right)^{(1)} / 24 \\
& A_{14}=\left(\widetilde{B}, C_{2}\right)^{(3)} / 36 \text {, } \\
& \left.\left.\left.A_{34}=[\widetilde{D}, \widetilde{D})^{(2)}, D_{2}\right)^{(1)}, \widetilde{K}\right)^{(1)}, D_{2}\right)^{(1)} / 64 \\
& \left.\left.\left.A_{35}=[\widetilde{D}, \widetilde{D})^{(2)}, \widetilde{E}\right)^{(1)}, D_{2}\right)^{(1)}, D_{2}\right)^{(1)} / 128 \text {, } \\
& A_{15}=(\widetilde{E}, \widetilde{F})^{(2)} / 4 \\
& \left.\left.A_{36}=[\widetilde{D}, \widetilde{E})^{(2)}, \widetilde{D}\right)^{(1)}, \widetilde{H}\right)^{(2)} / 16 \text {, } \\
& \left.\left.\left.\left.A_{16}=\left[\widetilde{E}, D_{2}\right)^{(1)}, C_{2}\right)^{(1)}, \widetilde{K}\right)^{(2)} / 16, \quad A_{37}=[\widetilde{D}, \widetilde{D})^{(2)}, \widetilde{D}\right)^{(1)}, \widetilde{D}\right)^{(3)} / 576 \text {, } \\
& \left.\left.\left.\left.\left.A_{17}=[\widetilde{D}, \widetilde{D})^{(2)}, D_{2}\right)^{(1)}, D_{2}\right)^{(1)} / 64, \quad A_{38}=\left[C_{2}, \widetilde{D}\right)^{(2)}, \widetilde{D}\right)^{(2)}, \widetilde{D}\right)^{(1)}, \widetilde{H}\right)^{(2)} / 64 \text {, } \\
& \left.A_{18}=[\widetilde{D}, \widetilde{F})^{(2)}, D_{2}\right)^{(1)} / 16 \\
& \left.A_{19}=[\widetilde{D}, \widetilde{D})^{(2)}, \widetilde{H}\right)^{(2)} / 16 \text {, } \\
& \left.A_{20}=\left[C_{2}, \widetilde{D}\right)^{(2)}, \widetilde{F}\right)^{(2)} / 16 \text {, } \\
& \left.\left.A_{39}=[\widetilde{D}, \widetilde{D})^{(2)}, \widetilde{F}\right)^{(1)}, \widetilde{H}\right)^{(2)} / 64 \text {, } \\
& \left.\left.A_{40}=[\widetilde{D}, \widetilde{D})^{(2)}, \widetilde{F}\right)^{(1)}, \widetilde{K}\right)^{(2)} / 64 \text {, } \\
& \left.\left.\left.A_{41}=\left[C_{2}, \widetilde{D}\right)^{(2)}, \widetilde{D}\right)^{(2)}, \widetilde{F}\right)^{(1)}, D_{2}\right)^{(1)} / 64 \text {, } \\
& \left.A_{21}=[\widetilde{D}, \widetilde{D})^{(2)}, \widetilde{K}\right)^{(2)} / 16 \text {, } \\
& \left.\left.A_{42}=[\widetilde{D}, \widetilde{F})^{(2)}, \widetilde{F}\right)^{(1)}, D_{2}\right)^{(1)} / 16 \text {. }
\end{aligned}
$$

In the above list, the bracket "[" is used in order to avoid placing the otherwise necessary up to five parenthesizes "(".

Finally we construct the needed affine invariants

$$
\begin{aligned}
\mathcal{F}_{1}(\tilde{a})= & A_{2}, \\
\mathcal{F}_{2}(\tilde{a})= & -2 A_{1}^{2} A_{3}+2 A_{5}\left(5 A_{8}+3 A_{9}\right)+A_{3}\left(A_{8}-3 A_{10}+3 A_{11}+A_{12}\right)- \\
& -A_{4}\left(10 A_{8}-3 A_{9}+5 A_{10}+5 A_{11}+5 A_{12}\right), \\
\mathcal{F}_{3}(\tilde{a})= & -10 A_{1}^{2} A_{3}+2 A_{5}\left(A_{8}-A_{9}\right)-A_{4}\left(2 A_{8}+A_{9}+A_{10}+A_{11}+A_{12}\right)+ \\
& +A_{3}\left(5 A_{8}+A_{10}-A_{11}+5 A_{12}\right), \\
\mathcal{F}_{4}(\tilde{a})= & 20 A_{1}^{2} A_{2}-A_{2}\left(7 A_{8}-4 A_{9}+A_{10}+A_{11}+7 A_{12}\right)+A_{1}\left(6 A_{14}-22 A_{15}\right)- \\
& -4 A_{33}+4 A_{34}, \\
\mathcal{F}(\tilde{a})= & A_{7}, \\
\mathcal{B}(\tilde{a})= & -\left(3 A_{8}+2 A_{9}+A_{10}+A_{11}+A_{12}\right), \\
\mathcal{H}(\tilde{a})= & -\left(A_{4}+2 A_{5}\right), \\
\mathcal{G}(\tilde{a}, x, y)= & \widetilde{M}+32 \widetilde{H},
\end{aligned}
$$

as well as the needed $C T$-comitants:

$$
\begin{aligned}
\mathcal{B}_{1}(\tilde{a})= & \left(T_{7}, D_{2}\right)^{(1)}\left[12 D_{1} T_{3}+2 D_{1}^{3}+9 D_{1} T_{4}+36\left(T_{1}, D_{2}\right)^{(1)}\right] \\
& \left.-2 D_{1}\left(T_{6}, D_{2}\right)^{(1)}\left[D_{1}^{2}+12 T_{3}\right]+D_{1}^{2}\left[D_{1}\left(T_{8}, C_{1}\right)^{(2)}+6\left(\left(T_{6}, C_{1}\right)^{(1)}, D_{2}\right)^{(1)}\right]\right\} / 144,
\end{aligned}
$$




$$
\begin{aligned}
\mathcal{B}_{2}(\tilde{a})= & \left\{\left(T_{7}, D_{2}\right)^{(1)}\left[8 T_{3}\left(T_{6}, D_{2}\right)^{(1)}-D_{1}^{2}\left(T_{8}, C_{1}\right)^{(2)}-4 D_{1}\left(\left(T_{6}, C_{1}\right)^{(1)}, D_{2}\right)^{(1)}\right]+\right. \\
& \left.+\left[\left(T_{7}, D_{2}\right)^{(1)}\right]^{2}\left(8 T_{3}-3 T_{4}+2 D_{1}^{2}\right)\right\} / 384, \\
\mathcal{B}_{3}(\tilde{a}, x, y)= & -D_{1}^{2}\left(4 D_{2}^{2}+T_{8}+4 T_{9}\right)+3 D_{1} D_{2}\left(T_{6}+4 T_{7}\right)-24 T_{3}\left(D_{2}^{2}-T_{9}\right), \\
\mathcal{B}_{4}(\tilde{a}, x, y)= & D_{1}\left(T_{5}+2 D_{2} C_{1}\right)-3 C_{2}\left(D_{1}^{2}+2 T_{3}\right) .
\end{aligned}
$$

2.2. Invariant polynomials associated to finite and infinite singularities of quadratic systems. In order to classify the family of systems possessing an integrable saddle we need the conditions for the number and multiplicities of the finite singularities of quadratic systems first constructed in [7].

We shall use the notion of zero-cycle of the complex plane in order to describe the number and multiplicity of singular points of a quadratic system. This notion is used for classification purposes of planar quadratic differential systems by Pal and Schlomiuk [19], Llibre and Schlomiuk [16], Schlomiuk and Vulpe [25] and by Artes, Llibre and Schlomiuk [2].

Definition 2. We consider formal expressions $\mathcal{D}=\sum n(w) w$ where $n(w)$ is an integer and only a finite number of $n(w)$ are nonzero. Such an expression is called a zero-cycle of $\mathbf{P}_{2}(\mathbb{C})$ if all $w$ appearing in $\mathcal{D}$ are points of $\mathbf{P}_{2}(\mathbb{C})$. We call degree of the zero-cycle $\mathcal{D}$ the integer $\operatorname{deg}(\mathcal{D})=\sum n(w)$. We call support of $\mathcal{D}$ the set $\operatorname{Supp}(\mathcal{D})$ of $w$ 's appearing in $\mathcal{D}$ such that $n(w) \neq 0$.

We note that $\mathbf{P}_{2}(\mathbb{C})$ denotes the complex projective space of dimension 2 . For a system $(S)$ belonging to the family (7) we denote $\nu(P, Q)=\left\{w \in \mathbb{C}_{2} \mid P(w)=Q(w)=0\right\}$ and we define the following zero-cycle $\mathcal{D}_{S}(P, Q)=\sum_{w \in \nu(P, Q)} I_{w}(P, Q) w$, where $I_{w}(P, Q)$ is the intersection number or multiplicity of intersection at $w$. It is clear that for a non-degenerate quadratic system $\operatorname{deg}\left(\mathcal{D}_{S}\right) \leq 4$ as well as $\# \operatorname{Supp}\left(\mathcal{D}_{S}\right) \leq 4$. For a degenerate system the zero-cycle $\mathcal{D}_{S}(P, Q)$ is undefined.

Using the invariant polynomials $\mu_{i}(i=0,1, \ldots, 4)$ in [32] the invariant polynomials $\mathbf{D}, \mathbf{P}$, $\mathbf{R}, \mathbf{S}, \mathbf{T}, \mathbf{U}, \mathbf{V}$, which are responsible for the number and multiplicities of finite singularities of a non-degenerate quadratic system are constructed. We note that these polynomials were constructed (using another way) and applied in [6, 7] (see also [3]). In [32] they are constructed as follows:

$$
\begin{aligned}
\mathbf{D}(\tilde{a}) & =\left[3\left(\left(\mu_{3}, \mu_{3}\right)^{(2)}, \mu_{2}\right)^{(2)}-\left(6 \mu_{0} \mu_{4}-3 \mu_{1} \mu_{3}+\mu_{2}^{2}, \mu_{4}\right)^{(4)}\right] / 48, \\
\mathbf{P}(\tilde{a}, x, y) & =12 \mu_{0} \mu_{4}-3 \mu_{1} \mu_{3}+\mu_{2}^{2}, \\
\mathbf{R}(\tilde{a}, x, y) & =3 \mu_{1}^{2}-8 \mu_{0} \mu_{2}, \\
\mathbf{S}(\tilde{a}, x, y) & =\mathbf{R}^{2}-16 \mu_{0}^{2} \mathbf{P}, \\
\mathbf{T}(\tilde{a}, x, y) & =18 \mu_{0}^{2}\left(3 \mu_{3}^{2}-8 \mu_{2} \mu_{4}\right)+2 \mu_{0}\left(2 \mu_{2}^{3}-9 \mu_{1} \mu_{2} \mu_{3}+27 \mu_{1}^{2} \mu_{4}\right)-\mathbf{P R}, \\
\mathbf{U}(\tilde{a}, x, y) & =\mu_{3}^{2}-4 \mu_{2} \mu_{4}, \\
\mathbf{V}(\tilde{a}, x, y) & =\mu_{4} .
\end{aligned}
$$

The geometrical meaning of the invariant polynomials above is revealed in the next proposition:

Proposition 1. ( [7]) The form of the divisor $\mathcal{D}_{S}(P, Q)$ for non-degenerate quadratic systems (7) is determined by the corresponding conditions indicated in Table 4, where we write $p+q+$ $r^{c}+s^{c}$ if two of the finite points, i.e. $r^{c}, s^{c}$, are complex but not real.

Regarding the expressions (12) we make the following remark:

Remark 2. If $\mu_{0}=0$ then the condition $\mathbf{R}=0$ (respectively $\mathbf{R}=\mathbf{P}=0 ; \mathbf{R}=\mathbf{P}=\mathbf{U}=0$; $\mathbf{R}=\mathbf{P}=\mathbf{U}=\mathbf{V}=0$ ) is equivalent to $\mu_{1}=0$ (respectively $\mu_{1}=\mu_{2}=0 ; \mu_{1}=\mu_{2}=\mu_{3}=0 ;$ $\mu_{1}=\mu_{2}=\mu_{3}=\mu_{4}=0$ ). 


\begin{tabular}{|c|c|c|c|c|c|}
\hline No & $\begin{array}{c}\text { Zero-cycle } \\
\mathcal{D}_{S}(P, Q) \\
\end{array}$ & $\begin{array}{l}\text { Invariant } \\
\text { criteria }\end{array}$ & No. & $\begin{array}{c}\text { Zero-cycle } \\
\mathcal{D}_{S}(P, Q) \\
\end{array}$ & $\begin{array}{l}\text { Invariant } \\
\text { criteria }\end{array}$ \\
\hline 1 & $p+q+r+s$ & $\begin{array}{c}\mu_{0} \neq 0, \mathbf{D}<0 \\
\mathbf{R}>0, \mathbf{S}>0\end{array}$ & 10 & $p+q+r$ & $\mu_{0}=0, \mathbf{D}<0, \mathbf{R} \neq 0$ \\
\hline 2 & $p+q+r^{c}+s^{c}$ & $\mu_{0} \neq 0, \mathbf{D}>0$ & 11 & $p+q^{c}+r^{c}$ & $\mu_{0}=0, \mathbf{D}>0, \mathbf{R} \neq 0$ \\
\hline \multirow{2}{*}{3} & \multirow{2}{*}{$p^{c}+q^{c}+r^{c}+s^{c}$} & $\mu_{0} \neq 0, \mathbf{D}<0, \mathbf{R} \leq 0$ & \multirow{2}{*}{12} & \multirow{2}{*}{$2 p+q$} & \multirow{2}{*}{$\mu_{0}=\mathbf{D}=0, \mathbf{P R} \neq 0$} \\
\hline & & $\mu_{0} \neq 0, \mathbf{D}<0, \mathbf{S} \leq 0$ & & & \\
\hline 4 & $2 p+q+r$ & $\mu_{0} \neq 0, \mathbf{D}=0, \mathbf{T}<0$ & 13 & $3 p$ & $\mu_{0}=\mathbf{D}=\mathbf{P}=0, \mathbf{R} \neq 0$ \\
\hline 5 & $2 p+q^{c}+r^{c}$ & $\mu_{0} \neq 0, \mathbf{D}=0, \mathbf{T}>0$ & 14 & $p+q$ & $\begin{array}{c}\mu_{0}=\mathbf{R}=0, \mathbf{P} \neq 0 \\
\mathbf{U}>0\end{array}$ \\
\hline 6 & $2 p+2 q$ & $\begin{array}{c}\mu_{0} \neq 0, \mathbf{D}=\mathbf{T}=0 \\
\mathbf{P R}>0\end{array}$ & 15 & $p^{c}+q^{c}$ & $\begin{array}{c}\mu_{0}=\mathbf{R}=0, \mathbf{P} \neq 0 \\
\mathbf{U}<0\end{array}$ \\
\hline 7 & $2 p^{c}+2 q^{c}$ & $\begin{array}{c}\mu_{0} \neq 0, \mathbf{D}=\mathbf{T}=0 \\
\mathbf{P R}<0\end{array}$ & 16 & $2 p$ & $\begin{array}{c}\mu_{0}=\mathbf{R}=0, \mathbf{P} \neq 0 \\
\mathbf{U}=0\end{array}$ \\
\hline 8 & $3 p+q$ & $\begin{array}{c}\mu_{0} \neq 0, \mathbf{D}=\mathbf{T}=0 \\
\mathbf{P}=0, \mathbf{R} \neq 0\end{array}$ & 17 & $p$ & $\begin{aligned} \mu_{0}= & \mathbf{R}=\mathbf{P}=0 \\
\mathbf{U} & \neq 0\end{aligned}$ \\
\hline 9 & $4 p$ & $\begin{array}{c}\mu_{0} \neq 0, \mathbf{D}=\mathbf{T}=0 \\
\mathbf{P}=\mathbf{R}=0\end{array}$ & 18 & 0 & $\begin{array}{c}\mu_{0}=\mathbf{R}=\mathbf{P}=0 \\
\mathbf{U}=0, \mathbf{V} \neq 0\end{array}$ \\
\hline
\end{tabular}

TABLE 4. The conditions for the number, multiplicities and types (real or/and complex) of the finite singularities of the generic family of Quadratic systems

The invariant polynomials which are responsible for the types of the finite singularities are constructed in paper [3]. Here we shall need the following ones (we keep the respective notations):

$$
\begin{aligned}
W_{1}(\tilde{a})= & {\left[A_{1}^{2}\left(A_{4}-2 A_{5}\right)-4 A_{1} A_{16}-4 A_{5} A_{8}-2 A_{4}\left(3 A_{8}+2 A_{11}\right)+4 A_{6}\left(A_{7}+7 A_{6}\right)\right] / 2 } \\
W_{2}(\tilde{a})= & {\left[44 A_{1} A_{2} A_{6}-23 A_{2}^{2} A_{4}-72 A_{3}\left(A_{19}+A_{21}\right)+72 A_{5}\left(A_{17}+4 A_{19}\right)\right.} \\
& \left.+A_{4}\left(14 A_{20}-50 A_{17}-32 A_{18}-75 A_{19}+93 A_{21}\right)-288 A_{6} A_{15}\right] / 9 \\
W_{3}(\tilde{a})= & {\left[A_{1}^{2}\left(36 A_{18}-19 A_{2}^{2}+134 A_{17}+165 A_{19}\right)+3 A_{11}\left(42 A_{18}-102 A_{17}+195 A_{19}\right)\right.} \\
& +2 A_{2}^{2}\left(A_{10}+3 A_{11}\right)+102 A_{3}\left(3 A_{30}-14 A_{29}\right)-63 A_{6}\left(17 A_{25}+30 A_{26}\right) \\
& +3 A_{10}\left(14 A_{18}-118 A_{17}+153 A_{19}+120 A_{21}\right)+6 A_{7}\left(329 A_{25}-108 A_{26}\right) \\
& +3 A_{8}\left(164 A_{18}+153 A_{19}-442 A_{17}\right)+9 A_{12}\left(2 A_{20}-160 A_{17}-2 A_{18}-59 A_{19}\right) \\
& +3 A_{1}\left(77 A_{2} A_{14}+235 A_{2} A_{15}-54 A_{36}\right)+18 A_{21}\left(21 A_{9}-5 A_{11}\right)+302 A_{2} A_{34} \\
& \left.-366 A_{14}^{2}-12 A_{15}\left(71 A_{14}+80 A_{15}\right)\right] / 9 \\
& +6 A_{2}^{2}\left(23 A_{21}-1093 A_{19}\right)-87 A_{2}^{4}+4 A_{2}^{2}\left(61 A_{17}+52 A_{18}+11 A_{20}\right) \\
& -6 A_{37}\left(352 A_{3}+939 A_{4}-1578 A_{5}\right)-36 A_{8}\left(396 A_{29}+265 A_{30}\right) \\
& +72 A_{29}\left(17 A_{12}-38 A_{9}-109 A_{11}\right)+12 A_{30}\left(76 A_{9}-189 A_{10}-273 A_{11}-651 A_{12}\right) \\
& -648 A_{14}\left(23 A_{25}+5 A_{26}\right)-24 A_{18}\left(3 A_{20}+31 A_{17}\right)+36 A_{19}\left(63 A_{20}+478 A_{21}\right) \\
& +18 A_{21}\left(2 A_{20}+137 A_{21}\right)-4 A_{17}\left(158 A_{17}+30 A_{20}+87 A_{21}\right) \\
& \left.-18 A_{19}\left(238 A_{17}+669 A_{19}\right)\right] / 81, \\
W_{4}(\tilde{a})= & 1512 A_{10}^{2}\left(A_{30}-2 A_{29}\right)-648 A_{15} A_{26}+72 A_{1}\left(49 A_{25}+39 A_{26}\right) \\
E_{3}(\tilde{a})= & 2 A_{1}^{2}-A_{8}-A_{12}, \\
G_{8}(\tilde{a})= & \left.-18 A_{1}^{2}+5 A_{8}-A_{10}-3 A_{11}+9 A_{12}\right) / 2, \\
G_{9}(\tilde{a})= & \left(A_{4}+2 A_{5}\right) / 4, \\
G_{10}(\tilde{a})= & A_{26} \cdot \\
& +48 A_{37}\left(A_{1}^{2}-A_{8}-A_{12}\right), \\
W_{7}(\tilde{a})= & \mu_{26}(\tilde{a}, x, y) \\
&
\end{aligned}
$$


Besides the invariant polynomials associated to finite singularities we need here the polynomials which are responsible for the number, multiplicities and the types of infinite singularities. Such polynomials as well as the respective affine invariant conditions are constructed in [25]. In order to detect directly the phase portrait of the systems with four invariant lines (considered with multiplicity and including the line of infinity) we shall also apply the respective affine invariant conditions constructed in [27]. Moreover we shall use also the affine invariant polynomials constructed in [5] to distinguish some phase portraits.

Here we shall apply only the following invariant polynomials (we keep the respective notations from [25] [27] and [5]):

$$
\begin{aligned}
\widetilde{M}(\tilde{a}, x, y) & =2 \operatorname{Hess}\left(C_{2}(\tilde{a}, x, y)\right) ; \\
\eta(\tilde{a}) & =\operatorname{Discrim}\left(C_{2}(\tilde{a}, x, y)\right) ; \\
\kappa(\tilde{a}) & =(\widetilde{M}, \widetilde{K})^{(2)} ; \\
\kappa_{1}(\tilde{a}) & =\left(\widetilde{M}, C_{1}\right)^{(2)} ; \\
\widetilde{N}(\tilde{a}, x, y) & =4 \widetilde{K}-4 \widetilde{H} ;
\end{aligned}
$$

$$
\begin{aligned}
& \tilde{L}(\tilde{a}, x, y)=16 \widetilde{K}-32 \widetilde{H}-\widetilde{M} \\
& K_{1}(\tilde{a}, x, y)=p_{1}(x, y) q_{2}(x, y)-p_{2}(x, y) q_{1}(x, y) \\
& K_{2}(\tilde{a}, x, y)=4\left(T_{2}, \widetilde{M}-8 \widetilde{K}\right)^{(1)}+3 D_{1}\left(C_{1}, \widetilde{M}-8 \widetilde{K}\right)^{(1)}-(\widetilde{M}-8 \widetilde{K})\left(16 T_{3}+3 T_{4} / 2+3 D_{1}^{2}\right) ; \\
& \kappa(\tilde{a})=(\widetilde{M}, \widetilde{K})^{(2)} ; \\
& \kappa_{1}(\tilde{a})=\left(\widetilde{M}, C_{1}\right)^{(2)} ; \\
& B_{3}(\tilde{a}, x, y)=\left(C_{2}, \widetilde{D}\right)^{(1)}=\operatorname{Jacob}\left(C_{2}, \widetilde{D}\right) \text {, } \\
& B_{2}(\tilde{a}, x, y)=\left(B_{3}, B_{3}\right)^{(2)}-6 B_{3}\left(C_{2}, \widetilde{D}\right)^{(3)} \text {, } \\
& B_{1}(\tilde{a})=\operatorname{Res}_{x}\left(C_{2}, \widetilde{D}\right) / y^{9}=-2^{-9} 3^{-8}\left(B_{2}, B_{3}\right)^{(4)}, \\
& B_{4}(\tilde{a}, x, y)=-\left((\widetilde{D}, \widetilde{H})^{(2)}, \widetilde{H}\right)^{(1)} \times(\widetilde{D}, \widetilde{H})^{(2)}, \\
& B_{5}(\tilde{a}, x, y)=D_{2}\left[\left(\left(C_{2}, D_{2}\right)^{(1)}, D_{2}\right)^{(1)}-3\left(C_{2}, \widetilde{K}\right)^{(2)}\right] \text {, } \\
& H_{1}(\tilde{a})=-\left(\left(\left(C_{2}, C_{2}\right)^{(2)}, C_{2}\right)^{(1)}, \widetilde{D}\right)^{(3)} ; \\
& H_{6}(\tilde{a}, x, y)=16 \widetilde{N}^{2}\left(C_{2}, \widetilde{D}\right)^{(2)}+H_{2}^{2}\left(C_{2}, C_{2}\right)^{(2)} ; \\
& H_{7}(\tilde{a})=\left(\tilde{N}, C_{1}\right)^{(2)} \\
& H_{9}(\tilde{a})=-\left(\left((\widetilde{D}, \widetilde{D})^{(2)}, \widetilde{D},\right)^{(1)} \widetilde{D}\right)^{(3)} \text {; } \\
& H_{10}(\tilde{a})=\left((\widetilde{N}, \widetilde{D})^{(2)}, D_{2}\right)^{(1)} ; \\
& H_{11}(\tilde{a}, x, y)=-32 \widetilde{H}\left[\left(C_{2}, \widetilde{D}\right)^{(2)}+8\left(\widetilde{D}, D_{2}\right)^{(1)}\right]+3 H_{2}^{2} \text {; } \\
& H_{12}(\tilde{a}, x, y)=(\widetilde{D}, \widetilde{D})^{(2)} \text {, } \\
& N_{1}(\tilde{a}, x, y)=C_{1}\left(C_{2}, C_{2}\right)^{(2)}-2 C_{2}\left(C_{1}, C_{2}\right)^{(2)}, \\
& N_{2}(\tilde{a}, x, y)=D_{1}\left(C_{1}, C_{2}\right)^{(2)}-\left(\left(C_{2}, C_{2}\right)^{(2)}, C_{0}\right)^{(1)} \text {, } \\
& N_{3}(\tilde{a}, x, y)=\left(C_{2}, C_{1}\right)^{(1)} \text {. }
\end{aligned}
$$

\section{The Proof of Main Theorem}

According to [10] (see also, [14]) if a system (7) possesses a weak saddle then via an affine transformation this system can be brought to the form (5) with the weak weak saddle $M_{0}(0,0)$. For these systems Cai Sui Lin has calculated the first three dual Poincaré-Lyapunov constants (6). 
So in order to have an integrable saddle the conditions $L_{1}^{*}=L_{2}^{*}=L_{3}^{*}=0$ have to be satisfied. We shall consider two cases: $b^{2}+l^{2} \neq 0$ and $b=l=0$.

3.1. The case $b^{2}+l^{2} \neq 0$. Without loss of generality we may assume $b \neq 0$ due to the change $(x, y, t, a, b, c, k, l, m) \mapsto(y, x,-t, m, l, k, c, b, a)$. Moreover we may consider $b=1$ due to the rescaling $y \rightarrow y / b$ and then the condition $L_{1}^{*}=l m-a=0$ gives $a=l m$. Herein we calculate

$$
\begin{aligned}
L_{2}^{*} & =\left(k-c l^{3}\right)(2+m)(2 m-1), \\
L_{3}^{*} & =(l-c k)\left(k-c l^{3}\right) m(2 m-1), \\
\mathcal{T}_{4} & \left.=0, \quad \mathcal{T}_{3}=\left(k-c l^{3}\right)(2 m-1)\right)^{3}, \\
\mathcal{T}_{2} & =(2 m-1)^{2}\left(2 k+3 c k l+l^{2}+2 c l^{3}+k m+c l^{3} m-l^{2} m^{2}\right) \\
\mathcal{T}_{1} & =\left(k-c l^{3}\right)(2 m-1)(1+2 m), \quad \sigma=(2 m-1)(l x-y)
\end{aligned}
$$

and we shall force the condition $L_{2}^{*}=0$.

3.1.1. The subcase $\mathcal{T}_{3} \neq 0$. Then we have $\left(k-c l^{3}\right)(2 m-1) \neq 0$ and the condition $L_{2}^{*}=0$ gives $m=-2$ and then $L_{3}^{*}=10(l-c k)\left(k-c l^{3}\right)=0$. As $\mathcal{T}_{3} \neq 0\left(\right.$ i.e. $\left.k \neq c l^{3}\right)$ we obtain $l=c k$ and this leads to the family of systems

$$
\dot{x}=x-2 c k x^{2}+x y+c y^{2}, \quad \dot{y}=-y-k x^{2}-c k x y+2 y^{2},
$$

having the first integral $\Phi(x, y)=\mathcal{H}_{N}^{2} / \mathcal{H}_{D}^{3}$, where

$$
\begin{gathered}
\mathcal{H}_{N}(x, y)=k\left(1-c^{2} k\right)(x-c y)^{3}+3\left(1-c^{2} k\right)(c k x+y)(x-c y)+2 c(3 c k x+3 y-1), \\
\mathcal{H}_{D}(x, y)=1-2 c k x-2 y-k(x-c y)^{2}
\end{gathered}
$$

for which we calculate:

$$
\begin{gathered}
\mu_{0}=-2 k\left(1-c^{2} k\right)^{2}, \quad \widetilde{K}=2 k\left(1+2 c^{2} k\right) x^{2}-12 c k x y+2\left(2+c^{2} k\right) y^{2}, \\
\mathbf{D}=-5184 k\left(1+c^{2} k\right)^{2}, \quad \mathcal{T}_{3}=125 k\left(-1+c^{2} k\right)\left(1+c^{2} k\right) \neq 0, \\
W_{4}=4 k^{2}\left(1-c^{2} k\right)^{2}\left(1+c^{2} k\right)^{2}, \quad W_{3}=25 k^{2}\left(1-c^{2} k\right)^{2}\left(1+c^{2} k\right)^{2}, \\
W_{2}=42 k^{2}\left(1-c^{2} k\right)^{2}\left(1+c^{2} k\right)^{2}, \quad W_{1}=k^{2}\left(1-c^{2} k\right)^{2}\left(25-14 c^{2} k+25 c^{4} k^{2}\right) .
\end{gathered}
$$

We observe that the condition $\mathcal{T}_{3} \neq 0$ implies $\mu_{0} \mathbf{D} \neq 0$ and $W_{i}>0$ with $i=4,3,2$. We also observe the $W_{1}>0$ as Discrim $\left[\left(25-14 c^{2} k+25 c^{4} k^{2}\right), k\right]=-2304 c^{4} \leq 0$.

3.1.1.1. Assume first $\mu_{0}<0$. Then $\mathbf{D}<0$ and since we have already at least one singular point according to Table 4 we deduce that systems (14) have four real singular points. Moreover, in this case $\widetilde{K}>0$ and since $W_{i}>0$ for each $i=1,2,3,4$ according to [3, Table 1 , row 12] we get the configuration of finite singularities: $\$, n, n, n$.

To examine infinite singularities we calculate

$$
\eta=4 k\left(1-c^{2} k\right)^{2}, \quad \kappa=-112 k\left(1-c^{2} k\right)^{2}
$$

and as $k>0$ we obtain $\eta>0$ and $\kappa<0$. So according to [25] we obtain Fig.7, i.e. $S, S, N$. As a total result we obtain: $\$, n, n, n ; S, S, N \quad[(0,1,0,0,1 / 2,0),(0,0,-1,-1,0,2)]$

We claim that the parabola $\mathcal{H}_{D}(x, y)=0$ (see (15)) considered inside the Poincaré disk contains the saddle $(0,0)$ in its interior. Since $k>0$ we have

$$
\lim _{\|(x, y)\| \rightarrow \infty, x \not c y} \mathcal{H}_{D}(x, y)=-\infty, \quad \mathcal{H}_{D}(0,0)=1
$$

and this proves our claim. Since all the singularities are elementary the parabola must contain the infinite point and three finite ones which in this case are nodes. Ones the limits of the separatrices of the finite saddle are determined (i.e. they can not coincide with infinite separatrices) the phase portrait becomes Portrait 28 of Figure 1. 
3.1.1.2. Assume now $\mu_{0}>0$. Considering (16) we have $k<0$ and this implies $\mathbf{D}>0$. So systems (14) possess two real and two imaginary singular points. As $W_{4}>0$ according to [3, Table 1, row 23] we get the configuration of finite singularities: $\$, n$.

Since $\eta=-2 \mu_{0}<0$ following [25] we obtain Fig.30, i.e. only one real singularity, which is a node $N$. Therefore we have the configuration of singular points:

$\$, n ; N \quad[(0,1,0,0,1 / 2,0),(0,0,-1,1,0,2)]$

A phase portrait with this configuration of points could only be Portrait 31 (in case we have a loop a focus or a center must be inside the loop).

3.1.2. The subcase $\mathcal{T}_{3}=0, \mathcal{T}_{2} \neq 0$. Taking into account (13) the condition $L_{2}^{*}=0$ yields $k=c l^{3}$ and this implies $L_{3}^{*}=0$. In this case we have $\mathcal{T}_{2}=l^{2}(1+3 c l-m)(1+c l+m)(2 m-1)^{2} \neq 0$ that implies $l \neq 0$. Then we may assume $l=1$ due to the change $x \rightarrow x / l$ and $c \rightarrow c / l$ and this leads to the family of systems

$$
\dot{x}=x+m x^{2}+x y+c y^{2}, \quad \dot{y}=-y-c x^{2}-x y-m y^{2},
$$

for which we calculate:

$$
\begin{aligned}
\mathcal{T}_{2} & =(1+3 c-m)(1+c+m)(-1+2 m)^{2} \neq 0, \\
\mu_{0} & =(c-m)^{2}(-1+c+m)(1+c+m), \\
\widetilde{K} & =2(c-m)\left[x^{2}+2(c+m) x y+y^{2}\right], \\
\mathbf{D} & =48(1+3 c-m)^{3}(-1+c+m), \\
W_{4} & =-16(-1+2 c)^{4}(1+3 c-m)^{3}(1+c+m), \\
W_{3} & =-8(-1+2 c)^{2}(c-m)(1+3 c-m)^{2}(1+c+m)(-1+2 m)^{2} .
\end{aligned}
$$

If $\mu_{0} W_{4} \mathbf{D} \neq 0$ then the following relations hold:

$$
\operatorname{sign}\left(\mathbf{D} W_{4}\right)=-\operatorname{sign}\left(\mu_{0}\right) ; \quad \operatorname{sign}\left(\mathcal{T}_{2}\right)=-\operatorname{sign}\left(W_{4}\right) ;
$$

We shall consider three cases: $\mu_{0}<0, \mu_{0}>0$ and $\mu_{0}=0$.

3.1.2.1. Assume first $\mu_{0}<0$. As $\mu_{0}$ is the discriminant of the binary form $\widetilde{K}$ we have an invariant polynomial with the defined sing: $\operatorname{sign}(\widetilde{K})=\operatorname{sign}(c-m)$. Moreover as $\mu_{0} \mathcal{T}_{2} \neq 0$ by (19) the condition $\mathbf{D} \neq 0$ holds in this case.

3.1.2.1.1. If $\mathbf{D}<0$ according to Table 4 the systems possess four real singularities. As $\mu_{0}<0$ according to $(20)$ and (19) we have $W_{4} \leq 0$.

1) Assume $\widetilde{K}<0$ (i.e. $c-m<0$ ). According to [3, Table 1] systems (18) possess three saddles and one anti-saddle. We claim that in this case the condition $W_{4}<0$ holds. Indeed suppose that $W_{4}=0$. Then due to $\mathcal{T}_{2} \neq 0$ considering (19) we get $c=1 / 2$. Herein for systems (18) we obtain:

$$
\mu_{0}=1 / 16(-1+2 m)^{3}(3+2 m), \quad \widetilde{K}=(1-2 m)\left[x^{2}+(1+2 m) x y+y^{2}\right] .
$$

So the condition $\mu_{0}<0$ implies $-2 / 3<m<1 / 2$ and then $\widetilde{K}>0$. Hence the obtained contradiction proves our claim.

Thus $W_{4}<0$ and by [3] the anti-saddle must be either a focus or a center.

Remark 3. For systems (18) calculations yield:

$$
\mathcal{T}_{4}=\mathcal{T}_{3}=\mathcal{F}=\mathcal{F}_{1}=0, \quad \mathcal{T}_{2} \neq 0, \quad \mathcal{B}=-1 / 2(-1+2 m)^{4} .
$$

Considering (19) we get $\mathcal{B}<0$ and due to $W_{4}<0$ by (20) we have $\mathcal{T}_{2}>0$. Thus the conditions $\left(c_{5}\right)$ provided by Theorem 2 are verified.

So in the case under consideration (18) we have the configuration of finite singularities: $\$, c, s, s$. We shall consider infinite singularities. Calculations yield:

(21) $\quad \eta=(1-3 c+m)^{3}(1+c+m), \quad \kappa=16(c-m)(1-3 c+m)(1+c+m)(3 c+m-2)$

It is easy to check by means of linear relations that in the case under consideration the conditions $\eta>0$ and $\kappa>0$ hold. 
Next we observe that due to $\eta>0$ and $c-m<0$ we obtain $\operatorname{sign}(\kappa)=-\operatorname{sign}(m+3 c-2)$. Admitting that $\kappa<0$ we get $m+3 c-2>0$ and as $m-c>0$ after taking the sum we obtain $2(m+c-1)>0$ that contradicts $\mu_{0}<0$.

So, $\eta>0, \kappa>0$ and we have three simple real infinite singularities, which according to [25] are nodes (Fig.1). (Evidently these points must be nodes according to the index theory). Thus we have the configuration:

$$
\$, c, s, s ; N, N, N \quad[(0,1,0,1 / 3,1 / 2,0),(0,0,-1,0,-1 / 2,-1 / 3)] .
$$

In order to determine the respective phase portraits following [5] we calculate

$$
B_{1}=0, \quad B_{3} B_{5}=36 c(-1+c+m)(1+c+m)(-1+2 m)^{2}(x-y)^{2}(x+y)^{4}
$$

and due to $\mu_{0}<0$ we obtain $\operatorname{sign}\left(B_{3} B_{5}\right)=-\operatorname{sign}(c)$. So as $\eta>0, \kappa>0$ and $\sigma \neq 0$ according to [5] we get phase portrait $V u l_{9}$ if $B_{3} B_{5}<0 ; V u l_{8}$ if $B_{3} B_{5}>0$ and $V u l_{10}$ if $B_{3}=0$.

2) Assume now $\widetilde{K}>0$, i.e. $c-m>0$. According to [3, Table 1] systems (18) possess one saddle and three anti-saddles. We recall that in this case the condition $W_{4} \leq 0$ holds.

a) If $W_{4}<0$ then we have either a focus or a center. However taking into consideration Remark 3 we obtain a center. This leads to the configuration of finite singularities: $\$, c, n, n$.

$\boldsymbol{a}_{1}$ ) Suppose first $\eta>0$. It is easy to check by means of linear relations that in the case under consideration the condition $\eta \geq 0$ implies $\kappa<0$.

Thus in the case $\eta>0$ (then $\kappa<0$ ) according to [25] at infinity we have two saddles and one node (Fig.7). In such a way we get the configuration:

$$
\$, c, n, n ; S, S, N \quad[(0,1,0,-1 / 2,1 / 2,0),(0,0,-1,0,-1 / 2,1 / 2)] .
$$

On the other hand considering (22) by according to [5] we get phase portrait $V u l_{28}$ if $B_{3} B_{5}<$ $0 ; V u l_{26}$ if $B_{3} B_{5}>0$ and $V u l_{27}$ if $B_{3}=0$.

$\boldsymbol{a}_{2}$ ) In the case $\eta=0$ we get $m=3 c-1$ and then $\widetilde{M}=0, C_{2}=c(x+y)^{3}$ and $\mathcal{T}_{2}=$ $72 c(-1+2 c)^{2} \neq 0$. Therefore at infinity there exists a triple singular point which according to [25] is a saddle (Fig.34). So we arrive to the following configuration of singularities:

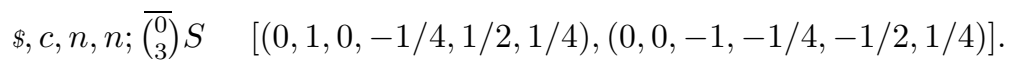

As there exists a center and $\mu_{0}<0, \mathbf{D}<0$ and $\eta=0$ by [5] we get the phase portrait given by $V u l_{25}$.

$\left.\boldsymbol{a}_{3}\right)$ Assume finally $\eta<0$. In this case at infinity we have two complex and one real singular point which due to $\mu_{0}<0$ is a saddle (see [25], Fig. 34). So we get the configuration

$\$, c, n, n ; S \quad[(0,1,0,-1,1 / 2,1),(0,0,-1,-1,-1 / 2,1)]$ and following [5] we arrive to the same Portrait Vul $_{25}$.

b) Admit now $W_{4}=0$. Considering (19) and the condition $\mathcal{T}_{2} \neq 0$ we get $c=1 / 2$. In this case for systems (18) we have

$$
\begin{aligned}
\mathcal{T}_{2} & =1 / 4(5-2 m)(-1+2 m)^{2}(3+2 m) \neq 0, \\
\mu_{0} & =1 / 16(-1+2 m)^{3}(3+2 m)=\eta, \\
\widetilde{K} & =(1-2 m)\left[x^{2}+(1+2 m) x y+y^{2}\right], \\
\mathbf{D} & =-3(-5+2 m)^{3}(-1+2 m), \\
W_{4} & =W_{3}=0, \quad W_{1}=1 / 32(-1+2 m)^{7}(3+2 m), \\
W_{2} & =1 / 16(-5+2 m)(-1+2 m)^{6}(3+2 m) .
\end{aligned}
$$

So the condition $\mu_{0}<0$ gives $-2 / 3<m<1 / 2$ (this implies $\mathbf{D}<0$ and $\widetilde{K}>0$ ) and then $W_{2}<0$. According to [3, Table 1] and Remark 3 besides the saddle systems (18) possess two nodes and one center. So we get the configuration of finite singularities $\$, c, n, n$.

As by (23) we have $\eta=\mu_{0}<0$, at infinity we have two complex and one real singular point which is a saddle (see [25], Fig. 34). So we get the configuration

$\$, c, n, n ; S \quad[(0,1,0,0,1 / 2,1 / 2),(0,0,-1,-1 / 2,-1 / 2,0)]$. 
It remains to note that we are still in the family defined by the conditions $\mu_{0}<0, \mathbf{D}<0$ and $\eta<0$. So by [5] we obtain again Portrait $V u l_{25}$.

3.1.2.1.2. Suppose $\mathbf{D}>0$, i.e. by Table 4 systems (18) possess two real and two complex finite singularities. It is easy to check by means of linear relations that in the case under consideration the condition $\mathbf{D}>0$ implies $\widetilde{K}<0$.

So $\mu_{0}<0, \widetilde{K}<0$ and according to [3, Table 1 , row 22] the two real points of systems (18) are saddles. To convince ourselves that both saddles are integrable we apply Theorem 2. Calculations yield: $\mathcal{H}=(m-c)(1+c+m)(-1+2 m)^{2}>0$ due to $\mu_{0}<0$ and $\widetilde{K}<0$. At the same time from $(20)$ we get $\mathcal{T}_{2}<0$ due to $W_{4}>0$. So the conditions $\left(c_{4}\right)$ of the Theorem 2 hold, i.e. we have two integrable saddles.

We shall examine infinite singularities. Considering (21) it was shown earlier (see page 18) that the conditions $\mu_{0}<0$ and $\widetilde{K}<0$ implies $\eta>0$ and $\kappa>0$. So by the same reasons, at infinity we get three nodes and we get the configuration

$$
\$, \$ ; N, N, N \quad[(0,1,0,1,1 / 2,-1),(0,0,-1,1,-1 / 2,-1)] .
$$

The existence of the invariant line $1+(m-c)(x+y)=0$ of systems (18) leads to the phase portrait given by Portrait $\mathrm{Ham}_{25}$.

3.1.2.2. Assume now $\mu_{0}>0$. According to (20) the condition $W_{4} \mathbf{D} \leq 0$ holds and we shall consider two cases: $\mathbf{D}<0$ and $\mathbf{D}>0$ (we recall that due to $\mu_{0} \mathcal{T}_{2} \neq 0$ we have $\mathbf{D} \neq 0$ ).

3.1.2.2.1. If $\mathbf{D}<0$ (this implies $W_{4} \geq 0$ ) according to [3] systems (18) have two saddles and two anti-saddles. Considering the graphics of the function $\mathbf{D}=0$ we observe that we have the following two disjoint domains where the conditions $\mu_{0}>0$ and $\mathbf{D}<0$ hold:

$$
\text { (i) } 1-m<c<(m-1) / 3 ; \quad(i i)(m-1) / 3<c<-m-1 .
$$

We claim that on these domains the second saddle is also an integrable saddle. Indeed, for systems (18) we have:

$$
\begin{aligned}
& \mathcal{T}_{4}=\mathcal{T}_{3}=\mathcal{F}=\mathcal{F}_{1}=0, \quad \mathcal{H}=(m-c)(1+c+m)(-1+2 m)^{2}, \\
& \mathcal{T}_{2}=(1+3 c-m)(1+c+m)(-1+2 m)^{2} \neq 0, \quad \mathcal{B}=-1 / 2(-1+2 m)^{4} .
\end{aligned}
$$

We observe that $\mathcal{B}<0$ (due to $\mathcal{T}_{2} \neq 0$ ). Moreover according to $(20)$ due to $W_{4}>0$ we have $\mathcal{T}_{2}<0$. It is easy to check by means of linear relations that on both domains (24) the condition $\mathcal{H}>0$ is verified. Therefore the conditions $\left(c_{4}\right)$ provided by Theorem 2 hold and this proves our claim.

So we have two integrable saddles and two anti-saddles. We claim that both anti-saddles of systems (18) must be nodes.

Indeed, as systems (18) possess three distinct singularities other than $M_{1}(0,0)$, by a linear transformation we can place two of these at the points $M_{2}(1,0)$ and $M_{3}(0,1)$. Without loss of generality we may assume that besides the integrable saddle $M_{1}$ the second such saddle is $M_{2}(1,0)$. Therefore following [3] we get the family of systems

$$
\dot{x}=c x+d y-c x^{2}+2 h x y-d y^{2}, \quad \dot{y}=e x-c y-e x^{2}+2 c x y+c y^{2},
$$

for which according to Theorem 2 the conditions $\left(c_{4}\right)$ must hold (in order to have two integrable saddles). Calculations yield:

$$
\begin{aligned}
& \mathcal{T}_{4}=\mathcal{T}_{3}=0, \quad \mathcal{T}_{2}=-4\left(c^{2}+d e\right)(c+h)^{2}\left(c^{2}-d e-2 e h\right)<0, \\
& \mathcal{F}_{1}=2(c+h)\left(2 c^{3}+c^{2} e+d e^{2}-2 c e h\right)=0, \quad \mathcal{B}=-2 e^{2}(c+h)^{4}<0 .
\end{aligned}
$$

As $\mathcal{B} \neq 0$ we have $e(c+h) \neq 0$ and we may assume $e=1$ due to a time rescaling. Then the condition $\mathcal{F}_{1}=0$ gives $d=-c\left(c+2 c^{2}-2 h\right)$ and therefore systems (26) besides the two integrable saddles possess the singularities $M_{3}(0,1)$ and $M_{4}(-2 c,-1)$ for which we have:

$$
\rho_{3}=2(c+h)=-\rho_{4}, \quad \delta_{3}=4\left(c+2 c^{2}-h\right)^{2}=\delta_{4} \geq 0 .
$$

So no one of anti-saddles could be point of the focus-center type and this proves our claim.

So both anti-saddles are nodes and we have the following configuration of finite singularities: $\$, \$, n, n$. 
On the other hand as $\mu_{0}>0$ According to [25] at infinity we obtain: two nodes and a saddle (Fig.5) if $\eta>0$ and one node (and two complex singularities, Fig. 30) if $\eta<0$.

If $\eta=0$ (i.e. $m=3 c-1$ ) we obtain $\widetilde{M}=0$ and at infinity there exists one real triple point, which is a node. So we get the configurations:

$$
\begin{aligned}
& \$, \$, n, n ; S, N, N \quad[(0,1,0,2,1 / 2,0),(0,0,-1,0,-1 / 2,-2)] \quad \text { if } \eta>0) ; \\
& \$, \$, n, n ; N \quad[(0,1,0,-2,1 / 2,-1 / 2),(0,0,-1,1 / 2,-1 / 2,2)] \quad(\text { if } \eta<0) ; \\
& \$, \$, n, n ; \overline{\left(\begin{array}{l}
0 \\
3
\end{array}\right) N} \quad[(0,1,0,-2,1 / 2,-1 / 3),(0,0,-1,1 / 3,-1 / 2,2)] \quad(\text { if } \eta=0) .
\end{aligned}
$$

We observe that systems (26) possess the invariant line $x-c y+c=0$ passing through the nodes $M_{3}(0,1)$ and $M_{4}(-2 c,-1)$ and through infinite singular point $N(1,-1,0)$. So it obviously could be seen that we obtain Portrait 29 if $\eta>0$ and Portrait 34 if $\eta \leq 0$.

3.1.2.2.2. Assume $\mathbf{D}>0$ (this implies $W_{4} \leq 0$ ). Then systems (18) possess two complex and two real singularities. More exactly, as $\mu_{0}>0$ according to [3] we have a saddle and an anti-saddle. It is not too difficult to detect that the conditions $\mu_{0}>0$ and $\mathbf{D}>0$ could be satisfied for and only for the points $(c, m)$ belonging to one of the domains:

$$
\text { (i) } 1-c<m<3 c+1 ; \quad \text { (ii) } 3 c+1<m<-c-1 .
$$

Considering the statement $(b)$ of Theorem 2 we deduce that the systems possess one integrable saddle and one center. Indeed the systems possess already an integrable saddle and by the mentioned theorem there must exist another weak singularity which could be only a center.

On the other hand as $\mu_{0}>0$ according to [25] at infinity we obtain: two nodes and a saddle (Fig.5) if $\eta>0$ and one node (and two complex singularities, Fig. 30) if $\eta<0$.

If $\eta=0$ (i.e. $m=3 c-1$ ) we obtain $\widetilde{M}=0$ and at infinity there exists one real triple point, which is a node. So we get the configurations:

$$
\begin{aligned}
& \$, c ; S, N, N \quad[(0,1,0,3,1 / 2,1),(0,0,-1,-1,-1 / 2,-3)] \quad(\text { if } \eta>0) \text {; } \\
& \$, c ; N \quad[(0,1,0,1 / 3,1 / 2,1),(0,0,-1,-1,-1 / 2,-1 / 3)] \quad(\text { if } \eta<0) \text {; } \\
& \$, c ; \overline{\left(\begin{array}{l}
0 \\
3
\end{array}\right)} N \quad[(0,1,0,2,1 / 2,1),(0,0,-1,-1,-1 / 2,-2)] \quad(\text { if } \eta=0) \text {; }
\end{aligned}
$$

On the other hand as $\mu_{0}>0$ and $\mathbf{D}>0$ according to [5] we obtain Portrait $V u l_{2}$ if $\eta \leq 0$ and Portrait $V u l_{19}$ if $\eta>0$.

3.1.2.3. Admit finally $\mu_{0}=0$. As $\mathcal{T}_{2} \neq 0$ from (19) we get $(c-m)(c+m-1)=0$.

3.1.2.3.1. If $\widetilde{K} \neq 0$ then $c-m \neq 0$ and we get $m=1-c$. So we get the family of systems

$$
\dot{x}=x+(1-c) x^{2}+x y+c y^{2}, \quad \dot{y}=-y-c x^{2}-x y-(1-c) y^{2},
$$

for which we calculate:

$$
\begin{aligned}
\mathcal{T}_{2} & =8 c(2 c-1)^{2} \neq 0, \quad \mathcal{T}_{4}=\mathcal{T}_{3}=\mathcal{F}=\mathcal{F}_{1}=0, \quad \mathbf{D}=\mathbf{R}=0, \\
\mathbf{P} & =4 c^{2}(x+y)^{4}, \quad \mathbf{U}=c^{2}(x-y)^{2}(x+y)^{4}, \quad G_{8}=4 c(2 c-1)^{2}, \\
\widetilde{K} & =2(2 c-1)(x+y)^{2}, \quad W_{4}=-2048 c^{3}(2 c-1)^{4}, \\
W_{3} & =-256 c^{2}(2 c-1)^{5}, \quad F_{2}=2 c(x+y)^{2} .
\end{aligned}
$$

As $\mathcal{T}_{2} \neq 0$ we have $W_{4} \mathbf{P} \neq 0$ and $\mathbf{U}>0$. According to Table 4 systems (29) possess two real finite singularities.

1) Assume first $W_{4}<0$. Then $c>0$ and this gives $F_{2}>0$ and $G_{8}>0$. As $\widetilde{K} \neq 0$ according to [3] besides the integrable saddle we have either a focus or a center. However by Theorem 2 in this case we must have two weak singularities and this leads to a center.

On the other hand in order to examine the infinite singularities in accordance with [25] we calculate:

$$
\eta=16(1-2 c)^{3}=\kappa / 4, \quad \mu_{0}=\mu_{1}=0, \quad \mu_{2}=2 c(x+y)^{2} .
$$

As $\mathcal{T}_{2} \neq 0$ we have $\eta \neq 0$. 
a) If $\eta>0$ (i.e. $0<c<1 / 2$ ) then systems possess 3 real distinct singularities and as $\kappa>0$ and $\mu_{2}>0$ by [25] at infinity we get Fig.5: two nodes and a saddle, and the saddle is a triple point (getting two finite singularities).

Thus we obtain the following configuration of singularities:

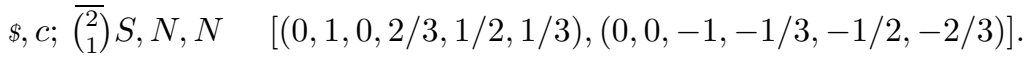

Considering the conditions $\mu_{0}=\mu_{1}=\mathbf{D}=0$ and $\eta>0$ according to [5] we arrive to the Portrait Vul $_{19}$.

b) Suppose $\eta<0$ (i.e. $c>1 / 2$ ). Then systems possess two imaginary and one real (triple) infinite singularities. As $\mu_{2}>0$ and $\kappa \neq 0$ according to [25] we have Fig.30 (one triple node which is semi-elementary). As a result we get the configuration

$$
\$, c ; \overline{\left(\begin{array}{l}
2 \\
1
\end{array}\right)} N \quad[(0,1,0,0,1 / 2,1),(0,0,-1,-1,-1 / 2,0)] .
$$

On the other hand since $\mu_{0}=\mu_{1}=0$ and the condition $\eta<0$ implies $\mu_{2}>0$, by [5] we obtain Portrait $\mathrm{Vul}_{2}$.

2) Admit now $W_{4}>0$, i.e. $c<0$. In this case considering (30) we obtain $\tilde{K}<0$ and $F_{2}<0$ and by [3, Table 1, row 148] the two real points of systems (18) are saddles. Clearly by Theorem 2 (see statement $(c)$ ) we must have two integrable saddles (as we have one at the origin of coordinates).

Indeed, for systems (29) for $c<0$ we have $\mathcal{T}_{2}<0, \mathcal{B}<0$ and $\mathcal{H}=2(1-2 c)^{3}>0$. So we arrive to the conditions $\left(c_{4}\right)$.

On the other hand for $c<0$ we obtain $\eta>0, \kappa>0$ and $\mu_{2}<0$. Therefore by [25] we have Fig.1, i.e. three nodes (one is triple). This leads to the configuration

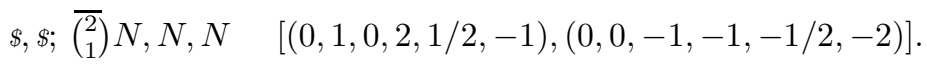

Taking into consideration the invariant line $(2 c-1)(x+y)=1$ which does not pass through the saddles we arrive to the phase portrait given by Portrait $\mathrm{Ham}_{25}$.

3.1.2.3.2. Assume finally $\widetilde{K}=0$. Then $m=c$ and we get the family of systems

$$
\dot{x}=x+c x^{2}+x y+c y^{2}, \quad \dot{y}=-y-c x^{2}-x y-c y^{2},
$$

for which we calculate:

$$
\begin{aligned}
\mathcal{T}_{2} & =(-1+2 c)^{2}(1+2 c)^{2}>0, \quad \mathcal{B}=-1 / 2(-1+2 c)^{4}, \\
\mu_{0} & =\mathbf{R}=0, \quad \mathbf{P}=(1+2 c)^{2}\left(c x^{2}+x y+c y^{2}\right)^{2}, \\
\mathbf{D} & =48(2 c-1)(1+2 c)^{3}, \quad \mathbf{U}=(x-y)^{2}\left(c x^{2}+x y+c y^{2}\right)^{2}, \\
W_{4} & =-16(-1+2 c)^{4}(1+2 c)^{4}, \quad G_{8}=1 / 2(-1+2 c)^{2}(1+2 c)^{2} .
\end{aligned}
$$

So by Table 4 we again have two real singularities (other two are gone to infinity). As $\widetilde{K}=0$, $W_{4}<0$ and $G_{8} \mathbf{D} \neq 0$, according to [3, Table 1] besides the integrable saddle systems (31) possess either a focus (row 157) or a center (row 162). However as by Theorem (2) the systems have to possess two weak singularities, we obtain a center. This can be deduced directly, as $\mathcal{T}_{2}>0$ and $\mathcal{B}=-(1-2 c)^{4} / 2<0$ (i.e. conditions $\left(c_{5}\right)$ are verified).

Next we calculate:

$$
\eta=(1-2 c)^{3}(1+2 c), \quad \mu_{2} \tilde{L}=8(2 c-1)(1+2 c)\left(c x^{2}+x y+c y^{2}\right)^{2}, \quad \kappa=0 .
$$

Due to $\mathcal{T}_{2} \neq 0$ we obtain $\eta \neq 0$ and $\operatorname{sign}(\eta)=-\operatorname{sign}\left(\mu_{2} \tilde{L}\right)$.

a) If $\eta>0$ then systems possess 3 real distinct singularities and as $\kappa=0$ and $\mu_{2} \tilde{L}<0$ by [25] at infinity we get Fig.3 (one node and two saddle-nodes). This leads to the following configuration of singularities $\$, c ; N, \overline{\left(\begin{array}{l}1 \\ 1\end{array}\right)} S N, \overline{\left(\begin{array}{l}1 \\ 1\end{array}\right)} S N \quad[(0,1,0,0,1 / 2,0),(0,0,-1,0,-1 / 2,0)]$.

In order to determine the respective phase portraits following [5] we calculate

$$
B_{3} B_{5}=3636 c(2 c-1)^{3}(1+2 c)(x-y)^{2}(x+y)^{4}
$$


and due to $\eta>0$ we obtain $\operatorname{sign}\left(B_{3} B_{5}\right)=-\operatorname{sign}(c)$. From $(32)$ we get $\operatorname{sign}(\mathbf{D})=-\operatorname{sign}(\eta)$ and therefore as $\eta>0$ (i.e. $\mathbf{D}<0$ ) according to [5] we obtain phase portrait $V u l_{18}$ if $B_{3} B_{5}<0$; $V u l_{16}$ if $B_{3} B_{5}>0$ and $V u l_{17}$ if $B_{3}=0$.

b) Assume now $\eta<0$. In this case due to $\kappa=0$ and $\mu_{2} \neq 0$ at infinity we get a simple node (imaginary points are both double ones) (see [25], Fig.30). So we have the configuration $\$, c ; N$ $[(0,1,0,1,1 / 2,1),(0,0,-1,-1,-1 / 2,-1)]$ and by $[5]$ in this case we get Portrait Vul $_{2}$.

3.1.3. The subcase $\mathcal{T}_{3}=\mathcal{T}_{2}=0, \sigma \neq 0$. Considering (13) the condition $\sigma \neq 0$ gives $2 m-1 \neq 0$ and then $\mathcal{T}_{3}=0$ implies $k=c l^{3}$. Herein we arrive to the family of systems

$$
\dot{x}=x+l m x^{2}+x y+c y^{2}, \quad \dot{y}=-y-c l^{3} x^{2}-l x y-m y^{2},
$$

for which we calculate:

$$
\begin{gathered}
\mathcal{T}_{2}=l^{2}(1+3 c l-m)(1+c l+m)(1-2 m)^{2}, \quad \sigma=(2 m-1)(l x-y), \\
\mu_{0}=l^{2}(c l-m)^{2}(-1+c l+m)(1+c l+m), \\
\mu_{1}=l(m-c l)(-1+c l+m)(1+2 m)(l x-y) .
\end{gathered}
$$

We shall consider two possibilities: $\mu_{0} \neq 0$ and $\mu_{0}=0$.

3.1.3.1. Assume first $\mu_{0} \neq 0$. Then $l \neq 0$ and we may assume $l=1$ due to the change $x \rightarrow x / l$ and $c \rightarrow c / l$. Therefore as $\sigma \neq 0$ (i.e. $2 m-1 \neq 0$ ) the condition $\mathcal{T}_{2}=0$ implies $m=1+3 c$. This leads to the family of systems

$$
\dot{x}=x+(1+3 c) x^{2}+x y+c y^{2}, \quad \dot{y}=-y-c x^{2}-x y-(1+3 c) y^{2},
$$

for which we calculate:

$$
\begin{aligned}
\mathcal{T}_{i} & =0(i=1,2,3,4), \quad \sigma=(1+6 c)(x-y) \neq 0, \quad \mathbf{D}=\mathbf{T}=\mathbf{P}=0, \\
\mu_{0} & =8 c(1+2 c)^{3} \neq 0, \quad \widetilde{K}=-2(1+2 c)\left[x^{2}+2(1+4 c) x y+y^{2}\right], \\
\mathbf{R} & =48 c^{2}(1+2 c)^{4}(x-y)^{2}, \quad E_{3}=-2 c(1+2 c)^{3}, \quad G_{10}=0 \\
\eta & =16(1+2 c), \quad \kappa=64(1+2 c)^{2}(1-6 c) .
\end{aligned}
$$

We observe that $\mu_{0} \neq 0$ implies $\mathbf{R} \neq 0$ and due to $\mathbf{D}=\mathbf{T}=\mathbf{P}=0$ according to Table 4 the systems above possess on triple and one simple real singularities. Considering (37) we have $\operatorname{sign}\left(E_{3}\right)=-\operatorname{sign}\left(\mu_{0}\right)$.

3.1.3.1.1. If $\mu_{0}<0$ (i.e. $-1 / 2<c<0$ ) we obtain $E_{3}>0$ and by [3, Table 1, row 94] besides the integrable saddle systems (36) possess a topological saddle. As the condition $\mu_{0}<0$ implies $\eta>0$ and $\kappa>0$, according to [25] at infinity we get Fig. 1 (three nodes). So we get the configuration: $\$, \bar{s}_{(3)} ; N, N, N \quad[(0,1,0,0,1 / 2,-1 / 3),(0,0,-1,1 / 3,-1 / 2,0)]$.

We observe that systems (36) possess as invariant curves a line and a conic:

$$
(2 c+1)(x+y)=1, \quad 1+4 c x+4 c y+2(2 c-1)\left(c x^{2}+2 x y+2 c x y+c y^{2}\right)=0
$$

Moreover both the curves pass through the triple singular point $M_{2}\left(x_{0}, y_{0}\right)$, where $x_{0}=y_{0}=$ $-1 /(4 c+2)$ (being tangent at this point). Thus in this case we obviously obtain Portrait $\operatorname{Ham}_{25}$.

3.1.3.1.2. Assume $\mu_{0}>0$, i.e. implies $c \in(-\infty,-1 / 2) \cup(0, \infty)$. In this case $E_{3}<0$ and besides the integrable saddle the systems possess an elliptic saddle (see [3, Table 1, row 88]).

1) If $\eta>0$ (i.e. $c>0$ ) by [25] at infinity we obtain Fig. 5 (two nodes and a saddle) and we get the configuration $\$, \widehat{e s}_{(3)} ; S, N, N \quad[(0,1,0,4,1 / 2,1),(0,0,-1,-1,-1 / 2,-4)]$.

Taking into account that the the invariant line (38) passes through infinite saddle $(1,-1,0)$ we arrive to Portrait 36.

2) Assuming $\eta<0$ (i.e. $c<-1 / 2$ ) by [25] at infinity we obtain a node (Fig.30). So we get the configuration $\$, \widehat{e s}_{(3)} ; N \quad[(0,1,0,-2,1 / 2,-1),(0,0,-1,1,-1 / 2,2)]$.

In this case the infinite singularity $(1,-1,0)$ is a node and this leads to the Portrait 35.

3.1.3.2. Suppose now $\mu_{0}=0$. This means that at least one finite singular point is gone to infinity. And the number of such points is governed by the invariant polynomials $\mu_{i}(\mathrm{i}=1,2,3)$. 
3.1.3.2.1. If $\mu_{1} \neq 0$ then considering (35) we have $l \neq 0$ and we may assume $l=1$ (due to the change $x \rightarrow x / l$ and $c \rightarrow c / l)$. So as $\sigma \neq 0$ the conditions $\mathcal{T}_{2}=\mu_{0}=0$ evidently yield $m=-1-c$ and this leads to the family of systems

$$
\dot{x}=x-(1+c) x^{2}+x y+c y^{2}, \quad \dot{y}=-y-c x^{2}-x y+(1+c) y^{2},
$$

for which we calculate:

$$
\begin{aligned}
& \mathcal{T}_{i}=0(i=1,2,3,4), \quad \sigma=(3+2 c)(y-x) \neq 0, \quad \mu_{0}=\eta=\kappa=0=G_{9}, \\
& \mathbf{D}=-768(1+2 c)^{3}, \quad \mu_{1}=2(1+2 c)^{2}(y-x), \quad W_{7}=12(1-2 c)^{4}(1+2 c)^{4}, \\
& \widetilde{K}=2(1+2 c)(x-y)^{2}, \quad \tilde{M}=-32 c^{2}(x-y)^{2}, \quad C_{2}=c(x-y)^{2}(x+y), \\
& \mathbf{R}=3 \mu_{1}^{2} \neq 0, \tilde{L}=-16 c(x-y)^{2}, \quad \widetilde{N}=(1-2 c)(1+2 c)(x-y)^{2} .
\end{aligned}
$$

1) If $\mathbf{D}<0$ then $c>-1 / 2$ and since $\mathbf{R} \neq 0$ by Table 4 the systems above possess three real simple singularities. We observe that in this case $\widetilde{K}>0, G_{9}=0$ and $W_{7} \geq 0$. Thus according to [3, Table 1, row 117] systems (39) besides the integrable saddle possess two nodes.

a) Assume $C_{2} \neq 0$. Then $c \neq 0$ and this implies $M \neq 0$. As $\eta=0, \mu_{1} \neq 0$ and $\kappa=0$, in order to determine the behavior of the trajectories at infinity, according to [25] we need the signs of the invariant polynomials $\tilde{L}, \widetilde{K}$ and $\widetilde{N}$.

Considering (40) we obtain:

$$
\operatorname{sign}(\widetilde{K})=-\operatorname{sign}(\mathbf{D}), \quad \operatorname{sign}(\tilde{L})=-\operatorname{sign}(c), \quad \operatorname{sign}(\tilde{N})=\operatorname{sign}\left(1-4 c^{2}\right) .
$$

$\boldsymbol{a}_{1}$ ) Admit first $\tilde{L}<0$, i.e. $c>0$. In this case the sign of $\tilde{N}$ determines the picture at infinity. Considering (40) we observe that the condition $W_{7} \neq 0$ implies $\widetilde{N} \neq 0$.

$\boldsymbol{\alpha}$ ) If $\tilde{N}>0$ (i.e. $0<c<1 / 2$ ) according to [25] we get Fig. 26, i.e. the configuration $S, \widehat{\left(\begin{array}{l}1 \\ 2\end{array}\right)} P E P-P H P$. So we arrive to the following configuration of all singularities:

$$
\$, n, n ; S, \widehat{\left(\begin{array}{l}
1 \\
2
\end{array}\right)} P E P-P H P \quad[(0,1,0,-5 / 4,1 / 2,1 / 4),(0,0,-1,-1 / 4,-1 / 2,5 / 4)] .
$$

We observe that systems (39) possess the invariant straight line $(2 c+1)(x+y)=1$ passing through the infinite saddle. Moreover on this line are located both finite nodes. Considering the type of the triple infinite point we obtain Portrait 38.

$\boldsymbol{\beta}$ ) In the case $\widetilde{N} \leq 0$ (i.e. $c \geq 1 / 2$ ) by [25] we get Fig. 16, i.e. the configuration $S, \widehat{\left(\begin{array}{c}1 \\ 2\end{array}\right)} P E P-$ $H$. So we get the configuration

$$
\$, n, n ; S, \widehat{\left(\begin{array}{l}
1 \\
2
\end{array}\right)} P E P-H \quad[(0,1,0,-2,1 / 2,1),(0,0,-1,-1,-1 / 2,2)] .
$$

In this case we apply the same arguments as above regarding the invariant line. And the type of the triple infinite point in this case corresponds to Fig. 16 and this produces the phase portrait given by Portrait $3 \%$.

$\boldsymbol{a}_{2}$ ) Suppose now $\tilde{L}>0$, i.e. $-1 / 2<c<0$. In this case $\widetilde{K}>0$ and according to [25] we obtain Fig. 23, i.e. we get the configuration $N, \widehat{\left(\begin{array}{l}1 \\ 2\end{array}\right)} H H H-H$. This leads to the final configuration of singularities

$$
\$, n, n ; N, \widehat{\left(\begin{array}{c}
1 \\
2
\end{array}\right)} H H H-H \quad[(0,1,0,-3 / 4,1 / 2,-1 / 4),(0,0,-1,1 / 4,-1 / 2,3 / 4)] .
$$

In this case the invariant line $(2 c+1)(x+y)=1$ passing through the infinite node and two finite nodes. Considering the type of the degenerate infinite point it must have three hyperbolic sector on the one side and one on the other side. Then applying the index theory (see [1, Proposition 4.8]) the finite saddle must be on the same side as the single infinite hyperbolic sector. Thus we get Portrait 39.

b) If $C_{2}=0$ we obtain $c=0$ (this implies $\widetilde{M}=0$ ) and we get the family of systems with infinite line filled up with singularities. In this case we have $\mu_{1}=2(y-x) \neq 0$ and according to $\left[29\right.$, Theorem $\left.7.1^{*}\right]$ in the neighborhood of infinity we get the behavior of the trajectories given by Fig. 41 (i.e. $(\infty, \emptyset)$ ). Considering the finite singularities we get

$\$, n, n ;(\infty, \emptyset) \quad[(0,1,0,-1,1 / 2,0),(0,0,-1,0,-1 / 2,1)]$. 
As for system (39) (with $c=0$ ) we have $H_{9}=-9216$ and $H_{10}=144$ according to [29, Theorem 3.3] we obtain the phase portrait Picture $C_{2} .1$ which corresponds to Portrait 40.

2) Admit now $\mathbf{D}>0$ then $c<-1 / 2$. As $\mathbf{R} \neq 0$ by Table 4 the systems above possess two imaginary singularities and one real (the integrable saddle). We note that this is in accordance with [3, Table 1 , row 123] as we have $\widetilde{K}<0$. So it remains to discuss only about infinite singularities.

Considering (40) in the case $c<-1 / 2$ we have $\tilde{L}>0$ and $\widetilde{K}<0$ and according to [25] we obtain Fig. 9. So we obtain the configuration of singularities

$$
\$ ; N, \widehat{\left(\begin{array}{c}
1 \\
2
\end{array}\right)} P E P-H \quad[(0,1,0,0,1 / 2,-1),(0,0,-1,1,-1 / 2,0)] .
$$

It obviously could be observed that this configuration leads to the phase portrait, which is topologically equivalent to $\mathrm{Ham}_{19}$.

3.1.3.2.2. Assume now $\mu_{1}=0$, i.e. two finite singularities are gone to infinity.

1) Suppose first $\mu_{2} \neq 0$. We claim that in this case for a non-degenerate system (34) the condition $l=0$ must hold. Indeed supposing the contrary, we may consider $l=1$ (due to a rescaling) and taking into account (35) and $\sigma \neq 0$ the conditions $\mathcal{T}_{2}=\mu_{0}=\mu_{1}=0$ implies either $c=-1 / 2=m$ or $c=0, m=1$ (the second case gives a degenerate system). However in both cases we get $\mu_{2}=0$ and this contradiction proves our claim.

So $l=0$ and we obtain the family of systems

$$
\dot{x}=x+x y+c y^{2}, \quad \dot{y}=-y-m y^{2},
$$

for which we calculate:

$$
\begin{aligned}
\mathcal{T}_{i} & =0(i=1,2,3,4), \quad \sigma=(1-2 m) y \neq 0, \quad \eta=\kappa=\kappa_{1}=0=G_{8}, \\
\mathbf{D} & =\mathbf{R}=0, \quad \mathbf{P}=(1-m)^{2} m^{2} y^{4}, \quad \mathbf{U}=y^{4}(-x+m x-c y)^{2}, \\
\widetilde{K} & =-2 m y^{2}, \quad \mu_{0}=\mu_{1}=0, \quad \mu_{2}=m(m-1) y^{2}=F_{2}, \\
\tilde{L} & =8 m(1+m) y^{2}, \quad \widetilde{M}=-8(1+m)^{2} y^{2}, \quad K_{2}=48\left(2+3 m+2 m^{2}\right) y^{2} .
\end{aligned}
$$

As $\mu_{2} \neq 0$ (then $\mathbf{P} \neq 0$ ) due to $\mathbf{U}>0$ by Table 4 the systems above have two real finite singularities. We shall examine two subcases: $\mu_{2}<0$ and $\mu_{2}>0$.

a) Assume first $\mu_{2}<0$. Then $0<m<1$ and this implies $\widetilde{K}<0$. As $F_{2}<0$ according to [3, Table 1, row 148] the second finite singular point is a saddle. By Theorem 2 this point could not be a weak saddle (according to statement $(e)$ a system in this class could have only one weak singularity). This fact could be established directly, as the trace corresponding to the second saddle equals $(2 m-1) / m \neq 0$ (due to $\sigma \neq 0$ ). So for the finite singularities we get the configuration $\$, s$.

Regarding the infinite singularities according to [25] we get Fig. 10 (as $\eta=0, \widetilde{M} \neq 0$, $\mu_{0}=\mu_{1}=\kappa=\kappa_{1}=0, \tilde{L}>0$ and $\left.\widetilde{K}<0\right)$, i.e. the configuration $N,\left(\begin{array}{l}2 \\ 2\end{array}\right) P E P-P E P$. So we get the configuration $\$, s ; N,\left(\begin{array}{l}2 \\ 2\end{array}\right) P E P-P E P \quad[(0,1,0,0,1 / 2,0),(0,0,-1,0,0,-1 / 2)]$.

Systems (42) possess two parallel lines $y=0$ and $y=-1 / m$ and both finite singularities are located on these lines (one on each line). We observe that if $c=0$ there appears one more invariant line $x=0$ passing through the finite singularities and this condition is given by invariant polynomial $B_{3}=-6 c y^{4}$. Taking into account the type of the degenerate singular point at infinity we obtain the phase portrait topologically equivalent to $\operatorname{Ham}_{20}$ if $B_{3} \neq 0$ and to $\mathrm{Ham}_{21}$ if $\mathrm{B}_{3}=0$.

b) If $\mu_{2}>0$ we have $m \in(-\infty, 0) \cup(1, \infty)$.

$\boldsymbol{b}_{1}$ ) Assume $\tilde{L}<0$, i.e. $-1<m<0$. As $\mu_{2}>0$ by [25] we get Fig. 17 (i.e. $S,\left(\begin{array}{l}2 \\ 2\end{array}\right) P E P-P E P$. This leads to the following configuration of singular points:

$$
\$, n ; S,\left(\begin{array}{l}
2 \\
2
\end{array}\right) P E P-P E P \quad[(0,1,0,0,1 / 2,1),(0,0,-1,0,0,1 / 4)] .
$$

Considering the parallel invariant lines mentioned above (which pass through the finite node and saddle as well as the infinite degenerate point) by the same reasons as above we arrive to Portrait 41 if $B_{3} \neq 0$ and Portrait 42 if $B_{3}=0$. 
$\boldsymbol{b}_{2}$ ) Supposing $\tilde{L}>0$ we obtain $m \in(-\infty,-1) \cup(1, \infty)$.

If $m>1$ we have $F_{2}>0$ and as $G_{8}=0$ by [3, Table 1, row 150] the second finite singularity of systems (42) is a node.

On the other hand considering the conditions $\tilde{L}>0$ and $K_{2}>0$, by [25] we arrive to Fig. $19\left(N,\left(\begin{array}{l}2 \\ 2\end{array}\right) P H-P H\right)$. However taking into consideration the existence of two invariant lines $(y=0$ and $y=-m)$ of systems (42) we obtain the configuration

$\$, n ; N,\left(\begin{array}{l}2 \\ 2\end{array}\right) P P H-P P H \quad[(0,1,0,0,1 / 2,0),(0,0,-1,0,0,-2)]$.

We observe that the parallel invariant lines split the three sectors at infinity. Then applying the index theory (see [1, Proposition 4.8]) both infinite hyperbolic sectors must be limited by the infinite line and the invariant line passing through the finite node. This leads to Portrait 43.

If $m<-1$ then considering (43) we observe that $K_{2}>0$ (as the discriminant of the binary form $2+3 m+2 m^{2}$ equals $\left.-7<0\right)$. Therefore by [25] we arrive again to Fig. $19\left(N,\left(\begin{array}{l}2 \\ 2\end{array}\right) P H-\right.$ $P H)$. So we obtain the same configuration as above:

$\$, n ; N,\left(\begin{array}{l}2 \\ 2\end{array}\right) P P H-P P H \quad[(0,1,0,0,1 / 2,0),(0,0,-1,0,0,2)]$.

$\boldsymbol{b}_{3}$ ) Admit now $\tilde{L}=0$. As $\mu_{2} \neq 0$ we get $m=-1$ and besides the integrable saddle $M_{1}(0,0)$ systems (42) possess the node $M_{2}(-c / 2,1)$. As $C_{2}=c y^{3}$ we shall consider two possibilities: $C_{2} \neq 0$ and $C_{2}=0$.

$\boldsymbol{\alpha}$ ) If $C_{2} \neq 0$ then due to $\widetilde{M}=0$ at infinity we have only one (real) singularity. As $\mu_{0}=\mu_{1}=0, \widetilde{K}=2 y^{2} \neq 0$ and $K_{2}=48 y^{2}>0$ according to [25] we obtain Fig. 32 $\left.\left(\begin{array}{l}2 \\ 3\end{array}\right) P E P H-P\right)$. So we obtain the configuration

$$
\$, n ;\left(\begin{array}{l}
2 \\
3
\end{array}\right) P E P H-P \quad[(0,1,0,0,1 / 2,1),(0,0,-1,0,0,1)] .
$$

In this case applying the index theory (see $[1$, Proposition 4.8$]$ ) we determine that the infinite hyperbolic sector must be bordered by the infinite line and the invariant line passing through the finite node. Then we obtain Portrait 44.

$\boldsymbol{\beta}$ ) Assuming $C_{2}=0$ (i.e. $c=0$ ) we get the system

$$
\dot{x}=x+x y, \quad \dot{y}=-y+y^{2},
$$

with infinite line filled up with the singularities. As $\mu_{1}=0, \mu_{2}=2 y^{2}>0$ and $K_{2}=2 y^{2}>0$ according to $\left[29\right.$, Theorem $\left.7.1^{*}\right]$ in the neighborhood of infinity we get the behavior of the trajectories given by Fig. 44, i.e. $(\infty, N)$. Considering the finite singularities we obtain the configuration $\$, n,(\infty, N)$. As for the system above we have $H_{10}=0, H_{11}=48 y^{4}$, $H_{12}=-32 y^{2}$ and $\mu_{2}=2 y^{2}$ according to [29, Theorem 3.3] we obtain the phase portrait Picture $C_{2} .5(b)$ which corresponds to Portrait 45.

2) Suppose finally $\mu_{2}=0$ and let us consider two subcases: $\widetilde{K} \neq 0$ and $\widetilde{K}=0$

a) If $\widetilde{K} \neq 0$ then the condition $l=0$ has to be satisfied, otherwise we get $l=1, c=-1 / 2=m$ and this implies $\widetilde{K}=0$. So we have $l=0$ and we consider the family (42). Taking into account (43) the condition $\mu_{2}=0$ due to $\widetilde{K} \neq 0$ gives $m=1$. Herein for the family (42) considering (43) we obtain

$$
\mathbf{R}=\mathbf{P}=\mu_{2}=0, \quad \mu_{3}=-c y^{3}, \quad \mathbf{U}=c^{2} y^{6}, \quad \widetilde{M}=-32 y^{2}, \quad \tilde{L}=16 y^{2}, \quad \widetilde{K}=-2 y^{2} .
$$

We observe that $c \neq 0$ (otherwise we get a degenerate system) and hence $\mu_{3} \mathbf{U} \neq 0$, i.e. by Table 4 the integrable saddle is a single singularity of systems (42).

On the other hand, at infinity we have two real singularities (due to $\widetilde{M} \neq 0$ ) and as $\kappa=\kappa_{1}=0$ (see (43)) and $\tilde{L}>0$ and $\widetilde{K}<0$ according to [25] we obtain Fig. $14\left(N,\left(\begin{array}{l}2 \\ 3\end{array}\right) P E H-P\right)$. So we obtain the configuration $\$ ; N,\left(\begin{array}{l}3 \\ 2\end{array}\right) P E P-P H \quad[(0,1,0,0,1 / 2,1),(0,0,-1,0,0,-1)]$.

Considering the invariant line $y=0$ on which lies the integrable saddle as well as the parallel invariant line $y=-1$ as well as the type of degenerate infinite singularity we obtain Portrait 46.

b) Admit now $\widetilde{K}=0$. 
$\boldsymbol{b}_{1}$ ) If $\tilde{L} \neq 0$ we have $l \neq 0$, otherwise setting $l=0$ we get systems (42), for which according to (43), the condition $\widetilde{K}=0$ implies $\tilde{L}=0$.

So $l \neq 0$ (then $l=1$ due to a rescaling) and it was shown earlier (see page 25) that in this case we get $c=-1 / 2=m$. Hence we obtain the system

$$
\dot{x}=x-x^{2} / 2+x y-y^{2} / 2, \quad \dot{y}=-y+x^{2} / 2-x y+y^{2} / 2,
$$

possessing a single singularity (the integrable saddle). For this system we calculate

$$
\begin{gathered}
\mu_{0}=\mu_{1}=\mu_{2}=\kappa=0, \quad \mu_{3}=-1 / 2(x-y)^{3}, \quad \tilde{L}=8(x-y)^{2}, \\
\eta=0, \quad \widetilde{M}=-8(x-y)^{2}, \quad \kappa_{1}=-64, \quad K_{1}=(x-y)^{3} / 2
\end{gathered}
$$

and as $\kappa_{1} \tilde{L} \neq 0$ and $\mu_{3} K_{1}=-(x-y)^{6} / 4<0$, according to [25] we obtain Fig. 9. This leads to the configuration of singularities

$$
\$ ; N,\left(\begin{array}{l}
3 \\
2
\end{array}\right) P E P-H \quad[(0,1,0,-1 / 2,1 / 2,-1 / 2),(0,0,-1,1 / 2,-1 / 2,1 / 2)] .
$$

In this case obviously we deduce that the phase portrait of the system is topologically equivalent to Ham $_{19}$.

$\boldsymbol{b}_{2}$ ) Assume now $\tilde{L}=0$. In this case clearly we must have $l=0$ and for systems (42) we get $m=0$. In this case evidently the saddle $M_{1}(0,0)$ is the unique finite singularity of these systems. Taking into account (43) we have

$$
\mu_{3}=-y^{2}(x+c y), \quad \widetilde{M}=-8 y^{2}, \quad \tilde{L}=\widetilde{K}=0, \quad K_{1}=y^{2}(x+c y) .
$$

As $\mu_{3} K_{1}=-y^{4}(x+c y)^{2}<0$ by [25] we get Fig. $12\left(\left(\begin{array}{l}1 \\ 1\end{array}\right) S N,\left(\begin{array}{l}2 \\ 2\end{array}\right) P E P-P E P\right)$. So we obtain the configuration $\$ ;\left(\begin{array}{l}1 \\ 1\end{array}\right) S N,\left(\begin{array}{l}2 \\ 2\end{array}\right) P E P-P E P \quad[(0,1,0,0,1 / 2,1),(0,0,-1,0,0,0)]$.

We observe that systems (42) with $l=m=0$ possess invariant line $y=0$ passing through the finite saddle and infinite degenerate point. Moreover the line $x=0$ also becomes invariant if and only $c=0$ (which is equivalent to $B_{3}=-6 c y^{4}=0$ ). So clearly we get Portrait 46 if $B_{3}=0$ and Portrait 47 if $B_{3} \neq 0$.

\subsubsection{The subcase $\sigma=0$ : Hamiltonian systems.}

Lemma 1. If a quadratic system (7) is Hamiltonian then the relation $\eta=-27 \mu_{0}$ is verified.

Proof: Clearly a system (7) is Hamiltonian if and only if $\partial P(x, y) / \partial x+\partial Q(x, y) / \partial y=0$ that gives $a_{10}+b_{01}=a_{20}+b_{11}=a_{11}+b_{02}=0$. Considering these relations a straightforward computation yield: $\eta+27 \mu_{0}=0$ and this completes the proof of the lemma.

Remark 4. It is known that a simple real point of a Hamiltonian system could be either a center or an integrable saddle. Therefore all the possibilities of the existence of an integrable saddle are described by the statement $(f)$ of Theorem 2. Moreover the respective conditions in fact are equivalent to the conditions for determining the number and multiplicity of finite singularities provided by Table 4 .

So taking into account this remark we shall consider step by step each of the set of conditions $\left(f_{1}\right)-\left(f_{6}\right)$, provided by the statement $(f)$ of Theorem 2 , in order to complete the respective configurations of the singularities. Moreover as the systems are Hamiltonian we shall apply directly the conditions constructed in [5] for the determination of the phase portraits, corresponding to this configuration of singularities.

3.1.4.1. The conditions $\left(f_{1}\right): \mu_{0}<0, \mathbf{D}<0, \mathbf{R}>0, \mathbf{S}>0$. By Theorem 2 in the finite part there are three integrable saddles and a center. As $\mu_{0}<0$ according to Lemma 1 we have $\eta>0$ and at infinity there exist three simple real singularities, which clearly are nodes. So we obtain the configuration: $\$, \$, \$, c ; N, N, N \quad[(0,1,0,-1,-1,0),(0,0,-1,0,1,1)]$.

Considering the conditions $\left(f_{1}\right)$ according to [5] we obtain the phase portrait $V u l_{11}$ if $B_{1} \neq 0$. In the case $B_{1}=0$ we get $V u l_{9}$ if $B_{3} B_{4}<0 ; V u l_{8}$ if $B_{3} B_{4}>0$ and $V u l_{10}$ if $B_{3}=0$. 
3.1.4.2. The conditions $\left(f_{2}\right): \mu_{0}>0, \mathbf{D}<0, \mathbf{R}>0, \mathbf{S}>0$. By Theorem 2 in the finite part there are two integrable saddles and two centers. As $\mu_{0}>0$ according to Lemma 1 we have $\eta<0$ and at infinity there exist two complex and one simple real singular point (a node). So we get the configuration: $\$, \$, c, c ; N \quad[(0,1,0,-1,-1,0),(0,-1,-1,1,1,1)]$. In this case by [5] we obtain $V u l_{4}$ if $B_{1} \neq 0$ and $V u l_{3}$ if $B_{1}=0$.

3.1.4.3. The conditions $\left(f_{3}\right): \mu_{0}=0, \mathbf{D}<0, \mathbf{R} \neq 0$. According to Table 4 a system of this class possesses three finite singularities. By Theorem 2 for Hamiltonian systems in this case there are two integrable saddles and one center. Following [4] a system from this family via an affine transformation and time rescaling could be brought to the form

$$
\dot{x}=c x-c x^{2}+d y, \quad \dot{y}=e x-e x^{2}-c y+2 c x y,
$$

for which we calculate:

$$
\begin{aligned}
& \mu_{0}=\eta=0, \quad \widetilde{M}=-72 c^{2} x^{2}, \quad \tilde{L}=24 c^{2} x^{2} \\
& \mathbf{D}=-192 c^{2} d^{2}\left(c^{2}-d e\right)^{2}\left(c^{2}+d e\right)^{2}, \quad \widetilde{K}=-4 c^{2} x^{2} .
\end{aligned}
$$

As $\mathbf{D}<0$ we have $c \neq 0$ and then $\widetilde{M} \neq 0, \tilde{L}>0$ and $\widetilde{K}<0$. So according to [25] at infinity we get Fig.9: $N,\left(\begin{array}{l}1 \\ 2\end{array}\right) P E P-H$. Therefore we arrive to the configuration of singularities $\$, \$, c ; N,\left(\begin{array}{l}1 \\ 2\end{array}\right) P E P-H \quad[(0,1,1,-1,0,0),(0,0,-1,0,1,0)]$.

According to [5] we obtain the phase portrait $V u l_{5}$ if $B_{1}=0$ and $V u l_{6}$ if $B_{1} \neq 0$.

3.1.4.4. The conditions $\left(f_{4}\right),[\alpha]: \mu_{0}<0, \mathbf{D}>0$. According to Table 4 a system of this class possesses two complex and two real finite singularities (which by Theorem 2 in this case are both integrable saddles). Considering Lemma 1 at infinity we have three simple singularities, which clearly are nodes. So we get the configuration of singularities

$\$, \$ ; N, N, N \quad[(0,0,0,0,-1,0),(1,0,0,-1,0,1)]$ and by [5] we obtain phase portrait $\operatorname{Ham}_{25}$ [5] if $B_{1} \neq 0$. In the case $B_{1}=0$ we get $H_{a m}$ if $B_{3} B_{4}>0$ and $H_{a m}$ if $B_{3} B_{4}>0$.

3.1.4.5. The conditions $\left(f_{4}\right),[\beta]: \mu_{0}<0, \mathbf{D}=0, \mathbf{T}<0$. According to Table 4 in this case we have one double and two simple real finite singularities. By Theorem 2 in this case both simple singular points are integrable saddles. As $\mu_{0}<0$ taking into account Lemma 1 at infinity we have three simple real point, which are nodes.

Thus it remains to detect the type of the double point. As we have two saddles, according to [3, Table 1, rows 80 and 81] the double point could be either a saddle-node, or a cusp (i.e. $\left.\widehat{c p}_{(2)}\right)$. Clearly in the class of Hamiltonian systems only the second possibility could be realizable.

By Lemma 1 the condition $\mu_{0}<0$ implies $\eta>0$ and we get the configuration of singularities $\$, \$, \widehat{c p}_{(2)} ; N, N, N \quad[(0,1,-1,-1,-1,1),(0,1,-1,-1,1,1)]$.

As $\mathbf{T} \neq 0$ according to [5] we obtain the phase portrait $\operatorname{Ham}_{27}$ if $B_{1} \neq 0$ and $\operatorname{Ham}_{24}$ if $B_{1}=0$.

3.1.4.6. The conditions $\left(f_{4}\right),[\gamma]: \mu_{0}=\mathbf{R}=0, \mathbf{P} \neq 0, \mathbf{U}>0, \widetilde{K} \neq 0$. By Table 4 a system from this family has two simple finite singularities, which by Theorem 2 are integrable saddles. As $K \neq 0$, in accordance with [4] we shall consider the respective canonical form:

$$
\dot{x}=c x+d y-c x^{2}+2 d u x y, \quad \dot{y}=e x+f y-e x^{2}+2 f u x y .
$$

For these systems $\widetilde{K}=4(d e-c f) u x^{2} \neq 0$ (i.e. $u \neq 0$ ) and hence in order to be Hamiltonian the conditions $f+c=d=u+1=0$ hold. Herein we calculate

$$
\begin{gathered}
\mu_{0}=\mu_{1}=\eta=\kappa=\kappa_{1}=0, \quad \mu_{2}=-c^{4} x^{2}, \quad \widetilde{M}=-72 c^{2} x^{2}, \\
\widetilde{K}=-4 c^{2} x^{2}, \quad \tilde{L}=24 c^{2} x^{2} .
\end{gathered}
$$

As $\widetilde{K} \neq 0$ we have $c \neq 0$ and then $\widetilde{M} \neq 0, \mu_{2}<0, \tilde{L}>0$ and $\widetilde{K}<0$. So according to [25] at infinity we get Fig.10: $N,\left(\begin{array}{l}2 \\ 2\end{array}\right) P E P-P E P$. So we arrive to the configuration of singularities $\$, \$ ; N,\left(\begin{array}{l}2 \\ 2\end{array}\right) P E P-P E P \quad[(0,1,0,-1,0,0),(0,0,-1,0,1,0)]$. As $\widetilde{M} \neq 0$ by [5] we obtain phase portraits $\mathrm{Ham}_{20}$ if $\mathrm{B}_{3} \neq 0$ and $\mathrm{Ham}_{21}$ if $B_{3}=0$. 
3.1.4.7. The conditions $\left(f_{5}\right),[\alpha]: \mu_{0}>0, \mathbf{D}>0$. Similarly as in the paragraph 3.1.4.4 above, on the finite part of phase plan we have two real and two complex singularities, but in this case by Theorem 2 we have a center and an integrable saddle. As $\mu_{0}>0$ by Lemma 1 we obtain $\eta<0$ and hence at infinity we have only one real point (a node) and two complex. So we arrive to the configuration $\$, c ; N \quad[(0,0,0,0,-1,0),(1,-3,0,2,0,1)]$ and according to [5] we obtain the phase portrait $\mathrm{Vul}_{2}$.

3.1.4.8. The conditions $\left(f_{5}\right),[\beta]: \mu_{0}>0, \mathbf{D}=0, \mathbf{T}<0$. According to Table 4 in this case we have one double and two simple real finite singularities. By Theorem 2 in this case we have an integrable saddle and a center. As $\mu_{0}>0$ taking into account Lemma 1 at infinity we have one simple real point (which is a node) and two complex singularities.

As for quadratic Hamiltonian systems the double point is a cusp we get the following configuration of singularities $\$, c, \widehat{c p}_{(2)} ; N, \quad[(0,-2,-4,2,2,4),(0,1,2,-1,-2,-2)]$ which leads to the phase portrait $\mathrm{Vul}_{7}$.

3.1.4.9. The conditions $\left(f_{5}\right),[\gamma]: \mu_{0}=\mathbf{R}=0, \mathbf{P} \neq 0, \mathbf{U}>0, \widetilde{K}=0$. By Table 4 a system from this family has two simple finite singularities. By Theorem 2 in this case we have an integrable saddle and a center. As $K=0$, in accordance with [4] we shall consider the respective canonical form:

$$
\dot{x}=\left(-g-k u^{2}\right) x-2 h y+g x^{2}+2 h x y+k y^{2}, \quad \dot{y}=u x+y .
$$

In order to be Hamiltonian the conditions $g=h=1-k u^{2}=0$ must hold. As $\mathbf{P}=k^{4} u^{4} y^{4} \neq 0$ we set $k=1 / u^{2}$ and then calculations yield:

$$
\mu_{0}=\mu_{1}=\eta=\kappa=\kappa_{1}=0, \quad \mu_{2}=y^{2} / u^{2}, \quad \widetilde{M}=0, \quad C_{2}=y^{3} / u^{2} .
$$

As $\widetilde{M}=0$ (i.e. at infinity there exists a single singular point) and $\mu_{2}>0$ according to [25] at infinity we get Fig.30 (in this case a nilpotent node of multiplicity five: $\widehat{\left(\begin{array}{l}2 \\ 3\end{array}\right)} N$ ). So we arrive to the configuration $\$, c ; \widehat{\left(\begin{array}{l}2 \\ 3\end{array}\right)} N \quad[(0,-1,0,0,0,1),(0,1,1,0,0,0)]$. On the other hand as $\widetilde{M}=0$ in the case under consideration according to [5] we obtain the phase portrait $V u l_{2}$.

3.1.4.10. The conditions $\left(f_{6}\right),[\alpha]: \mu_{0}<0, \mathbf{D}=\mathbf{T}=\mathbf{P}=0, \mathbf{R} \neq 0$. By Table 4 a system from this family has one triple and one simple finite singularities (and the simple one is an integrable saddle). As $\mu_{0}<0$ by Lemma 1 we obtain $\eta>0$, i.e. at infinity there are three real simple singularities.

In accordance with [4] we shall consider the respective family of systems, which in the case when the systems are Hamiltonian (after an additional rescaling) become of the form:

$$
\begin{aligned}
& \dot{x}=-u x-u^{2} y+u x^{2}+2\left(2 u^{2}-v\right) x y+u\left(4 u^{2}-3 v\right) y^{2}, \\
& \dot{y}=x+u y-x^{2}-2 u x y+\left(-2 u^{2}+v\right) y^{2} .
\end{aligned}
$$

For these systems we calculate

$$
\mu_{0}=4\left(u^{2}-v\right)^{3}<0, \quad \eta=-108\left(u^{2}-v\right)^{3}>0, \quad E_{3}=2\left(u^{2}-v\right)^{4},
$$

and as $E_{3}>0$ according to [3, Table 1, row 94] the triple point of systems above is topological saddle. So at infinity we have three nodes and we get the configuration

$$
\$, \bar{s}_{(3)} ; N, N, N \quad[(0,0,0,0,-1,0),(0,1,0,-1,0,1)] .
$$

On the other hand for systems (49) we have

$$
B_{1}=0, \quad B_{3} B_{4}=-5184\left(u^{2}-v\right)^{1} 0 y^{2}(x+u y)^{4}
$$

and as $\mu_{0}<0$ we obtain $B_{3} B_{4}<0$. So by [5] we get the phase portrait $\operatorname{Ham}_{25}$.

3.1.4.11. The conditions $\left(f_{6}\right),[\beta]: \mu_{0}=0, \mathbf{D}>0, \mathbf{R} \neq 0$. According to Table 4 we have one simple real (integrable saddle) and two complex singularities. Following [4] we shall consider the family of systems

$$
\begin{aligned}
& \dot{x}=2(h-g u) x+g\left(1+u^{2}\right) y+g x^{2}-2 h x y, \\
& \dot{y}=2(m-l u) x+l\left(1+u^{2}\right) y+l x^{2}-2 m x y,
\end{aligned}
$$


which are Hamiltonian if and only if $h=g-m=0$ and $l=2 m u /\left(1+u^{2}\right)$. For these systems we calculate

$$
\mu_{0}=\eta=\kappa=0, \mathbf{D}=12288 m^{12}\left(1+u^{2}\right)^{4}>0, \widetilde{M}=-72 m^{2} x^{2}=18 \widetilde{K}=-3 \tilde{L} .
$$

As $\mathbf{D}>0$ then $\widetilde{M} \neq 0, \tilde{L}>0$ and $\widetilde{K}<0$. So according to [25] at infinity we get Fig.9: $N, \widehat{\left(\begin{array}{c}1 \\ 2\end{array}\right)} P E P-H$ and this leads to the configuration of singularities

$\$ ; N, \widehat{\left(\begin{array}{c}1 \\ 2\end{array}\right)} P E P-H \quad[(0,0,1,1,0,0),(0,2,0,0,-1,0)]$. According to [5] we get in this case the phase portrait $\operatorname{Ham}_{19}$.

3.1.4.12. The conditions $\left(f_{6}\right),[\gamma]: \mu_{0}=0, \mathbf{D}=0, \mathbf{P R} \neq 0$. According to Table 4 we have one double and one simple real singular point (which is an integrable saddle). Taking into account [4] we shall consider the family of systems (with the additional rescaling $(x, y) \mapsto(x, u y)$ )

$$
\dot{x}=c x-c x^{2}+c y+2 h x y, \quad \dot{y}=e x-e x^{2}+e y+2 m x y,
$$

which are Hamiltonian if and only if $h=c-m=c+e=0$. For these systems we calculate

$$
\begin{gathered}
\mu_{0}=\eta=\kappa=\mathbf{D}=0, \quad \mu_{1}=-4 c^{4} x, \quad \mathbf{R}=48 c^{8} x^{2} \neq 0, \quad \widetilde{M}=-72 c^{2} x^{2}, \\
\tilde{L}=24 c^{2} x^{2}, \quad \widetilde{K}=-4 c^{2} x^{2}=\widetilde{H} .
\end{gathered}
$$

Due to $\mathbf{R} \neq 0$ we obtain $\mu_{1} \widetilde{M} \neq 0, \tilde{L}>0$ and $\widetilde{K}<0$. As we are in the class of Hamiltonian systems the double singular point of systems (51) is a cusp.

On the other hand, according to [25] at infinity we get Fig.9: $N, \widehat{\left(\begin{array}{l}1 \\ 2\end{array}\right)} P E P-H$. So we obtain the configuration $\$, \widehat{c p}_{(2)} ; N, \widehat{\left(\begin{array}{l}1 \\ 2\end{array}\right)} P E P-H \quad[(0,1,1,-1,0,0),(0,-1,-1,1,1,0)]$. As for Hamiltonian systems $(51)$ we have $B_{1}=-16 c^{1} 2 \neq 0$ by [5] in this case we get portrait $\operatorname{Ham}_{22}$.

3.1.4.13. The conditions $\left(f_{6}\right),[\delta]: \mu_{0}=\mathbf{R}=\mathbf{P}=0, \mathbf{U} \neq 0$. According to Table 4 we have a single finite singularity (the integrable saddle). According to [4] to this family of systems correspond two canonical forms: with $\widetilde{K} \neq 0$ and with $\widetilde{K}=0$. We claim that a system which verifies the conditions $\left(f_{6}\right),[\delta]$ and $\widetilde{K} \neq 0$ could not be Hamiltonian.

Indeed assume $\widetilde{K} \neq 0$. Then by [4] we should consider the family of systems (with the additional rescaling $(x, y) \mapsto(x, y / u))$

$$
\dot{x}=c x+d y+(2 c+d) x^{2}+2 d x y, \quad \dot{y}=e x+f y+(2 e+f) x^{2}+2 f x y,
$$

which are Hamiltonian if and only if $\sigma=c+f+2(2 c+d+f) x+2 d y=0$. However the last condition implies $d=c=f=0$ and this yields a degenerate system.

Thus it remains to examine the canonical form for this family, providing the additional condition $\widetilde{K}=0$ (see [4]):

$$
\dot{x}=x+d y, \quad \dot{y}=e x+l x^{2}+f y+2 m x y-d(l d-2 m) y^{2} .
$$

For these systems the conditions $\sigma=1+f+2 m x-2 d(l d-2 m) y=0$ gives $f=-1$ and $m=d=0$ (as $m=l=0$ leads to linear systems). Herein we calculate

$$
\mu_{0}=\mu_{1}=\mu_{2}=\eta=\kappa=0=\widetilde{M}, \quad \mu_{3}=-l x^{3} \neq 0, \quad C_{2}=-l x^{3}, \quad K_{1}=l x^{3} .
$$

As $\mu_{3} K_{1}=-l^{2} x^{6}<0$ according to [25] at infinity we get Fig.33: $\left(\begin{array}{l}3 \\ 3\end{array}\right) P E P E P-P$. Therefore we obtain the configuration $\$ ;\left(\begin{array}{l}3 \\ 3\end{array}\right) P E P E P-P \quad[(0,1,0,0,0,0),(0,0,-1,1,0,0)]$.

On the other hand, as the conditions $\mu_{0}=\mu_{1}=\mu_{2}=0$ and $\mu_{3} \neq 0$ hold, according to [5] in this case we obtain the phase portrait $\mathrm{Ham}_{18}$.

3.2. The case $b=l=0$. Then systems (5) become

$$
\dot{x}=x+a x^{2}+c y^{2}, \quad \dot{y}=-y-k x^{2}-m y^{2},
$$


for which we have $L_{1}^{*}=L_{2}^{*}=L_{3}^{*}=0$, i.e. the saddle $M_{1}(0,0)$ is integrable. On the other hand, for these family we calculate:

$$
\begin{aligned}
\mathcal{T}_{4} & =0, \quad \mathcal{T}_{3}=-8\left(a^{3} c-k m^{3}\right), \quad \mathcal{F}_{1}=\mathcal{F}_{2}=\mathcal{F}_{3} \mathcal{F}_{4}=0, \\
\eta & =-4 a^{3} c-27 c^{2} k^{2}+18 a c k m+a^{2} m^{2}-4 k m^{3}, \\
\mathbf{D} & =-48 \eta, \quad W_{4}=256 c^{2} k^{2} \eta, \quad \mu_{0}=(c k-a m)^{2}, \quad B_{3}=0 .
\end{aligned}
$$

Remark 5. According to [30] (see also [24] and [26]) if for a non-degenerate quadratic system with a finite number of infinite singularities the condition $B_{3}(a, x, y)=0$ holds in $\mathbb{R}[x, y]$, then this system possesses at least four invariant straight lines, considering the infinite line and their multiplicities.

According to this remark we shall use the affine invariant conditions given in [27] in order to determine the configuration of singularities as well as the phase portrait for a system in the family (54). For this propose we include here the needed affine invariant polynomial used in [27] (we keep here the respective notations adding the "hat"):

$$
\begin{aligned}
& \widehat{B}_{3}(\tilde{a}, x, y)=\left(C_{2}, \widetilde{D}\right)^{(1)}=\operatorname{Jacob}\left(C_{2}, \widetilde{D}\right), \\
& \widehat{B}_{2}(\tilde{a}, x, y)=\left(B_{3}, B_{3}\right)^{(2)}-6 B_{3}\left(C_{2}, \widetilde{D}\right)^{(3)} \text {, } \\
& \widehat{B}_{1}(\tilde{a})=\operatorname{Res}_{x}\left(C_{2}, \widetilde{D}\right) / y^{9}=-2^{-9} 3^{-8}\left(B_{2}, B_{3}\right)^{(4)}, \\
& \left.\widehat{H}_{1}(\tilde{a})=-\left(\left(C_{2}, C_{2}\right)^{(2)}, C_{2}\right)^{(1)}, \widetilde{D}\right)^{(3)} ; \\
& \widehat{H}_{2}(\tilde{a}, x, y)=\left(C_{1}, 2 H-N\right)^{(1)}-2 D_{1} N \text {; } \\
& \widehat{H}_{3}(\tilde{a}, x, y)=\left(C_{2}, \widetilde{D}\right)^{(2)} \text {; } \\
& \widehat{H}_{4}(\tilde{a})=\left(\left(C_{2}, \widetilde{D}\right)^{(2)},\left(C_{2}, D_{2}\right)^{(1)}\right)^{(2)} ; \\
& \widehat{H}_{5}(\tilde{a})=\left(\left(C_{2}, C_{2}\right)^{(2)},(\widetilde{D}, \widetilde{D})^{(2)}\right)^{(2)}+8\left(\left(C_{2}, \widetilde{D}\right)^{(2)},\left(\widetilde{D}, D_{2}\right)^{(1)}\right)^{(2)} \text {; } \\
& \widehat{H}_{6}(\tilde{a}, x, y)=16 N^{2}\left(C_{2}, \widetilde{D}\right)^{(2)}+H_{2}^{2}\left(C_{2}, C_{2}\right)^{(2)} \text {; } \\
& \widehat{H}_{7}(\tilde{a})=\left(N, C_{1}\right)^{(2)} \text {; } \\
& \widehat{H}_{8}(\tilde{a})=9\left(\left(C_{2}, \widetilde{D}\right)^{(2)},\left(\widetilde{D}, D_{2}\right)^{(1)}\right)^{(2)}+2\left[\left(C_{2}, \widetilde{D}\right)^{(3)}\right]^{2} ; \\
& \widehat{H}_{9}(\tilde{a})=-\left(\left((\widetilde{D}, \widetilde{D})^{(2)}, \widetilde{D},\right)^{(1)} \widetilde{D}\right)^{(3)} ; \\
& \widehat{H}_{10}(\tilde{a})=\left((N, \widetilde{D})^{(2)}, D_{2}\right)^{(1)} ; \\
& \widehat{H}_{11}(\tilde{a}, x, y)=8 H\left[\left(C_{2}, \widetilde{D}\right)^{(2)}+8\left(\widetilde{D}, D_{2}\right)^{(1)}\right]+3 H_{2}^{2} \text {; } \\
& \widehat{N}_{1}(\tilde{a}, x, y)=C_{1}\left(C_{2}, C_{2}\right)^{(2)}-2 C_{2}\left(C_{1}, C_{2}\right)^{(2)}, \\
& \widehat{N}_{2}(\tilde{a}, x, y)=D_{1}\left(C_{1}, C_{2}\right)^{(2)}-\left(\left(C_{2}, C_{2}\right)^{(2)}, C_{0}\right)^{(1)} \text {, } \\
& \widehat{N}_{3}(\tilde{a}, x, y)=\left(C_{2}, C_{1}\right)^{(1)} \text {, } \\
& \widehat{N}_{4}(\tilde{a}, x, y)=4\left(C_{2}, C_{0}\right)^{(1)}-3 C_{1} D_{1} \text {, } \\
& \widehat{N}_{5}(\tilde{a}, x, y)=\left[\left(D_{2}, C_{1}\right)^{(1)}+D_{1} D_{2}\right]^{2}-4\left(C_{2}, C_{2}\right)^{(2)}\left(C_{0}, D_{2}\right)^{(1)} \text {, } \\
& \widehat{N}_{6}(\tilde{a}, x, y)=8 \widetilde{D}+C_{2}\left[8\left(C_{0}, D_{2}\right)^{(1)}-3\left(C_{1}, C_{1}\right)^{(2)}+2 D_{1}^{2}\right] \text {. }
\end{aligned}
$$

3.2.1. The subcase $\mathcal{T}_{3} \neq 0$. We observe that if $\eta \neq 0$ (this implies $\mathbf{D} W_{4} \neq 0$ ) then

$$
\operatorname{sign}(\mathbf{D})=-\operatorname{sign}\left(W_{4}\right)=-\operatorname{sign}(\eta)
$$

As $\mu_{0} \geq 0$ we shall consider two subcases: $\mu_{0} \neq 0$ and $\mu_{0}=0$.

3.2.1.1. Assume first $\mu_{0} \neq 0$, i.e. $\mu_{0}>0$. 
3.2.1.1.1. If $\mathbf{D}<0$ according to Table 4 the systems possess four real singularities. As $\mathbf{D}<0$ we have $W_{4} \geq 0$.

1) If $W_{4} \neq 0$ (i.e. $W_{4}>0$ ) then $c k \neq 0$ and we may assume $c=k=1$ due to the rescaling $(x, y) \mapsto\left(c^{-1 / 3} k^{-2 / 3} x, c^{-2 / 3} k^{-1 / 3} y\right)$. In this case we get the family of systems

$$
\dot{x}=x+a x^{2}+y^{2}, \quad \dot{y}=-y-x^{2}-m y^{2} .
$$

As $\mu_{0}>0, \mathbf{D}<0$ and $W_{4}>0$ taking into account the fact that by Theorem 2 in the considered case we have only one weak singularity (which is saddle), according to [3, Table 1] systems (57) possess two saddles and either two nodes or two foci. We claim that the second case could not occur. Indeed, for systems above we have

$$
\begin{gathered}
\mathbf{D}=48\left(27+4 a^{3}-18 a m-a^{2} m^{2}+4 m^{3}\right)=-48 \eta, \quad \mu_{0}=(-1+a m)^{2}>0, \\
\mathcal{T}_{3}=8(m-a)\left(a^{2}+a m+m^{2}\right)=\mathcal{G}_{1}, \quad \widehat{B}_{3}=0, \quad \widehat{\theta}=64(1-a m), \quad \widehat{H}_{7}=-32 .
\end{gathered}
$$

According to [27] systems (57) subject to the conditions above possess the phase portrait Picture 4.1.(a) which is equivalent to our Portrait 29. Therefore they possess two saddles and two nodes on the phase plane and one saddle and two nodes at infinity: $\$, s, n, n ; S, N, N$ $[(0,1,0,-2,0,1),(0,0,-1,-1,0,2)]$.

2) Assume now $W_{4}=0$. As $\mathbf{D} \neq 0$ considering (55) we get $c k=0$ and without loss of generality we may assume $k=0$ due to the change $(x, y, t, a, c, k, m) \mapsto(y, x,-t, m, k, c, a)$. Moreover as as $\mu_{0}=a^{2} m^{2} \neq 0$ we may assume also $a=m=1$ due to the rescaling $(x, y) \mapsto$ $(x / a, y / m)$ and then we obtain

$$
\begin{gathered}
\mathcal{T}_{3}=-8 c \neq 0, \quad \mathbf{D}=48(4 c-1)=-48 \eta, \quad \mu_{0}=1, \\
\widetilde{N}=4 c y^{2}, \quad \widehat{B}_{3}=\widehat{\theta}=0, \quad \widehat{H}_{1}=-576(-1+4 c) .
\end{gathered}
$$

As $\mathbf{D}<0$ we have $c<1 / 4$ and $c \neq 0$ due to $\mathcal{T}_{3} \neq 0$ and this implies $\eta>0, \widetilde{N} \widehat{H}_{1} \neq 0$. So considering [28] we determine that the one parameter family of systems (57) in this case possess invariant lines of total multiplicity five. Moreover there exists a unique phase portrait given by Picture 5.1 (which is topologically equivalent to Portrait 29). Hence these systems possess two saddles and two nodes on the phase plane and one saddle and two nodes at infinity: $\$, s, n, n ; S, N, N \quad[(0,1,0,1,0,-1),(0,0,-1,0,0,-1)]$.

3.2.1.1.2. Suppose now $\mathbf{D}>0$. According to Table 4 the systems possess two real and two complex singularities. As $\mathbf{D}>0$ by (55) and (56) we have $W_{4} \leq 0$ and $\eta<0$.

1) If $W_{4} \neq 0$ (i.e. $\left.W_{4}<0\right)$ then $c k \neq 0$ and it was mentioned above that we may assume $c=k=1$ due to a rescaling. So we shall consider the family of systems (57).

By (58) the condition $\mathbf{D}>0$ implies $\eta<0$ and $\mu_{0} \mathcal{T}_{3} \neq 0$ gives $\widehat{\theta} \mathcal{G}_{1} \neq 0$. Therefore considering (58) according to [27] we get the phase portrait Picture 4.2.(a) which is equivalent to Portrait 30. So on the phase plane of systems (57) besides the integrable saddle there exists a focus and at at infinity we have a single real point which is a node. This leads to the configuration

$$
\$, f ; N \quad[(0,1,0,1,0,1),(0,0,-1,-1,0,0)] .
$$

2) Assuming $W_{4}=0$ we may assume (as above) $k=0$ and considering (59) due to $\mathbf{D}>0$ we have $c>1 / 4$. Then $\eta<0, \widehat{H}_{1} \widetilde{N} \neq 0$ and by [27] we obtain the phase portrait Picture 5.2 (equivalent to Portrait 31). So we get the following configuration of singularities

$$
\$, n ; N \quad[(0,1,0,1,0,0),(0,0,-1,-1,0,-2)] .
$$

3.2.1.1.3. Admit finally $\mathbf{D}=0$. Considering (55) we get $W_{4}=\eta=0$. We observe that the condition $\mathcal{T}_{3} \neq 0$ implies $c^{2}+k^{2} \neq 0$ and we could we may assume $k \neq 0$ due to the change $(x, y, t, a, c, k, m) \mapsto(y, x,-t, m, k, c, a)$. Moreover we may consider $k=1$ due to the rescaling $y \rightarrow k y$. Then the condition $\mathbf{D}=0$ yields

$$
0=27 c^{2}+2 a c\left(2 a^{2}-9 m\right)-\left(a^{2}-4 m\right) m^{2} \equiv \psi(a, c, m)
$$

and as $\psi(a, c, m)$ is quadratic in $c$ we must have Discrim $[\psi, c]=16\left(a^{2}-3 m\right)^{3} \geq 0$. So without loss of generality we could set a new parameter $u$ as follows: $a^{2}-3 m=u^{2}$. Then 
$m=\left(a^{2}-u^{2}\right) / 3$ and therefore we obtain

$$
\psi(a, c, m)=\bar{\psi}(a, c, u)=0 \quad \Rightarrow \quad c=\left(a^{3}-3 a u^{2}+2 \varepsilon u^{3}\right) / 27, \quad(\varepsilon= \pm 1) .
$$

Herein systems (54) become a 2-parameter family of systems

$$
\dot{x}=x+a x^{2}+\left(a^{3}-3 a u^{2}+2 \varepsilon u^{3}\right) y^{2} / 27, \quad \dot{y}=-y-x^{2}-\left(a^{2}-u^{2}\right) y^{2} / 3,
$$

for which we have $\mathcal{T}_{3}=-8 \varepsilon u^{3}(a-\varepsilon u)^{2}(2 a+\varepsilon u) / 27$. As $\mathcal{T}_{3} \neq 0$ we have $u \neq 0$ and due to the change $(x, y, a) \mapsto\left(x /(u \epsilon), y /(u \epsilon)^{2}\right.$, aut) we may assume $\varepsilon u=1$ (i.e. $\varepsilon=1=u$ ). Herein we obtain the systems

$$
\dot{x}=x+a x^{2}+(a-1)^{2}(a+2) y^{2} / 27, \quad \dot{y}=-y-x^{2}-\left(a^{2}-1\right) y^{2} / 3,
$$

for which we calculate:

$$
\begin{aligned}
\mathbf{D} & =\eta=W_{4}=0, \quad \mathcal{T}_{3}=-\frac{8}{27}(a-1)^{2}(2 a+1), \quad \widehat{\theta}=\frac{128}{729}(a-1)^{3}(2+a)(1+2 a)^{2}, \\
\mu_{0} & =\frac{4}{729}(a-1)^{2}(2 a+1)^{4}, \quad \widehat{H}_{7}=-\frac{32}{27}(-1+a)^{2}(2+a), \quad \widetilde{M}=-\frac{8}{9}(3 x-y+a y)^{2} .
\end{aligned}
$$

If $\widehat{\theta} \neq 0$ then $\widehat{H}_{7} \neq 0$ and according to [27] as $\mu_{0}>0$ we get Picture 4.25(a) which is equivalent to Portrait 32.

If $\widehat{\theta}=0$ due to $\mathcal{T}_{3} \neq 0$ we have $a=-2$ and for system (60) we obtain:

$$
\eta=0, \quad \widetilde{M}=-8(x-y)^{2}, \quad \mu_{0}=4, \quad \widehat{\theta}=\widehat{B}_{3}=0, \quad \widetilde{N}=4 x^{2}, \quad \widetilde{D}=-x(x-y)^{2} .
$$

So by [28] the phase portrait of this system is given by Picture 5.11 (equivalent to Portrait 32).

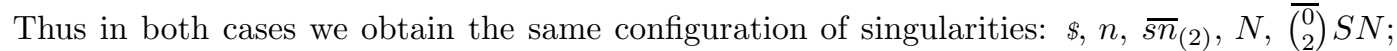
examples: $[a=0$ if $\widehat{\theta} \neq 0$ and $a=-2$ if $\widehat{\theta}=0]$.

3.2.1.2. Assume now $\mu_{0}=0$. It was shown above that due to $\mathcal{T}_{3} \neq 0$ we may assume $k=1$ and then the condition $\mu_{0}=(c-a m)^{2}=0$ gives $c=a m$. So we obtain the family of systems

$$
\dot{x}=x+a x^{2}+a m y^{2}, \quad \dot{y}=-y-x^{2}-m y^{2},
$$

for which calculations yield:

$$
\begin{gathered}
\mathcal{T}_{3}=-8\left(a^{2}-m\right) m\left(a^{2}+m\right)=\widehat{\mathcal{G}}_{1} \neq 0, \quad \eta=-4 m\left(a^{2}+m\right)^{2}, \\
\mu_{0}=\mu_{1}=0, \quad \mu_{2} \tilde{L}=8\left(a^{2}+m\right)^{2}\left(x^{2}+m y^{2}\right)^{2}, \quad \tilde{N}=4 m(x+a y)^{2}, \\
\widehat{\theta}=\widehat{B}_{3}=0, \quad W_{4}=-1024 a^{2} m^{3}\left(a^{2}+m\right)^{2}, \quad \widehat{H}_{7}=-32 a m, \\
\widehat{H}_{6}=8192 a m^{2} x y(x+a y)^{2}\left[\left(a^{2}-3 m\right) x^{2}-8 a m x y-\left(3 a^{2}-m\right) m y^{2}\right] .
\end{gathered}
$$

The condition $\mathcal{T}_{3} \neq 0$ implies $\eta \tilde{L} \widetilde{N} \widehat{\mathcal{G}}_{1} \mu_{2} \neq 0$ and then $\mu_{2} \tilde{L}>0$ and $\operatorname{sign}(\eta)=-\operatorname{sign}(m)$. Moreover $\widehat{H}_{7}=0$ (i.e. $a=0$ ) if and only if $\widehat{H}_{6}=0$ and this is equivalent to $W_{4}=0$. We shall consider two subcases: $\eta>0$ and $\eta<0$.

3.2.1.2.1. Assume $\eta>0$, i.e. $m<0$. Considering (63) according to [27] and [28] we obtain Picture $4.18(a)$ if $\widehat{H}_{7} \neq 0$ (i.e. $W_{4}>0$ ) and Picture 5.7 (which is equivalent to Picture 4.18(a) if $\widehat{H}_{7}=0$ (then $W_{4}=0$ ). Our corresponding phase portrait is Portrait 33. So in this case we get the configuration:

$\$, n, N, \overline{\left(\begin{array}{l}1 \\ 1\end{array}\right)} S N, \overline{\left(\begin{array}{l}1 \\ 1\end{array}\right)} S N$; examples: $\left[a=1, m=-2\right.$ if $W_{4} \neq 0 ; a=0, m=-1$ if $\left.W_{4}=0\right]$.

3.2.1.2.2. Suppose $\eta<0$, i.e. $m>0$. Similarly as above considering (63) according to [27] and [28] we obtain Picture 4.27(a) (which corresponds to Portrait 30) if $\widehat{H}_{7} \neq 0$ (i.e. $W_{4}<0$ ) and Picture 5.9 (equivalent to Portrait 31) if $\widehat{H}_{7}=0$ (i.e. $W_{4}=0$ ). So we get respectively the next configurations of singularities:
$\$, f ; N$
$[(0,1,0,1,0,2),(0,0,-1,-1,0,-2)] \quad\left(\right.$ if $\left.W_{4}<0\right)$;
$\$, n ; N$
$[(0,1,0,0,0,0),(0,0,-1,-1,0,-1)] \quad\left(\right.$ if $\left.W_{4}=0\right)$. 
3.2.2. The subcase $\mathcal{T}_{3}=0, \mathcal{T}_{2} \neq 0$. In this case for systems (54) the conditions

$$
\mathcal{T}_{3}=-8\left(a^{3} c-k m^{3}\right)=0, \quad \mathcal{T}_{2}=4\left(a^{3} c+3 a c k m-a^{2} m^{2}+k m^{3}\right) \neq 0
$$

have to be satisfied. We claim that these conditions imply am $\neq 0$. Indeed suppose the contrary, that $a m=0$. We observe that the condition $\mathcal{T}_{2} \neq 0$ implies $a^{2}+m^{2} \neq 0$ and we may assume $m \neq 0$ due to the change $(x, y, t, a, c, k, m) \mapsto(y, x,-t, m, k, c, a)$. Then $a=0$ and therefore the condition $\mathcal{T}_{3}=0$ gives $k=0$. However this implies $\mathcal{T}_{2}=0$ and the obtained contradiction proves our claim.

So $a m \neq 0$ and we may assume $a=m=1$ due to the rescaling $(x, y) \mapsto(x / a, y / m)$. Hence the condition $\mathcal{T}_{3}=0$ gives $k=c$ and we arrive to the family of systems

$$
\dot{x}=x+x^{2}+c y^{2}, \quad \dot{y}=-y-c x^{2}-y^{2},
$$

for which we calculate:

$$
\begin{gathered}
\mathcal{T}_{2}=4(1+c)(3 c-1) \neq 0, \quad \eta=-(1+c)(3 c-1)^{3}, \quad \mu_{0}=\left(c^{2}-1\right)^{2}, \\
\mathbf{D}=48(1+c)(3 c-1)^{3}, \quad W_{4}=-256 c^{4}(1+c)(3 c-1)^{3}, \quad \mathcal{F}_{1}=0 \\
\mathcal{B}=-8, \quad \mathcal{H}=4\left(1-c^{2}\right), \quad \widetilde{N}=4 c\left(x^{2}+2 c x y+y^{2}\right), \quad \widehat{B}_{3}=0=\widehat{\mathcal{G}}_{1} \\
\widehat{\theta}=64 c^{2}\left(c^{2}-1\right)^{2}, \quad \widehat{H}_{7}=-32 c^{2}, \quad \widehat{H}_{1}=576(1+c)(1-3 c)^{3} .
\end{gathered}
$$

3.2.2.1. Assume first $\mu_{0} \neq 0$, i.e. $\mu_{0}>0$. We observe that the condition $\mathcal{T}_{2} \neq 0$ implies $\eta \mathbf{D}<0$.

3.2.2.1.1. If $\mathbf{D}<0$ (i.e. $-1<c<1 / 3$ ) then according to Table 4 the systems possess four real singularities. As $\mu_{0}>0$ on the phase plane of systems (64) there are two saddles and two anti-saddles. Moreover as $\mathcal{F}_{1}=0 \mathcal{B}<0$ and for $-1<c<1 / 3$ we get $\mathcal{T}_{2}<0$ and $\mathcal{H}>0$ by Theorem 2 we conclude that both saddles are integrable.

We observe that in this case $W_{4} \geq 0$ and we shall consider two possibilities: $W_{4} \neq 0$ and $W_{4}=0$.

1) Assume $W_{4} \neq 0$ (i.e. $W_{4}>0$ ). Then $c \neq 0$ and considering (65) we have $\widehat{\theta} \widehat{H}_{7} \neq 0$ and according to [27] as $\eta>0$ and $\mu_{0}>0$ we get Picture 4.1(a) (equivalent to Portrait 29). So besides the two integrable saddle we have two nodes and at infinity we have one saddle and two nodes. So we obtain the configuration

$$
\$, \$, n, n ; S, N, N \quad[(0,1,0,1,0,-1 / 2),(0,0,-1,1 / 2,0,-1)] .
$$

2) Admitting $W_{4}=0$ (i.e. $c=0$ ) we get a concrete system (64) for which considering (65) we have $\widetilde{N}=\widehat{B}_{3}=0, \widehat{H}_{1}=576>0$ and as $\eta>0$ according to [28] the phase portrait of this system is given by Picture 6.1, which is topologically equivalent to Picture 4.1(a) (i.e. with Portrait 29). So we get the same configuration of singularities.

3.2.2.1.2. Assume now $\mathbf{D}>0$, i.e. $c \in(-\infty,-1) \cup(1 / 3, \infty))$. This implies $W_{4}<0$ and by [3, Table 1, rows 26 and 28] systems (64) possess one saddle (integrable) and either a focus or a center. As by Theorem 2 the systems must have two weak singularities (of the same degree of weakness) we obtain a center. It remains to observe that in this case $\eta<0, \mu_{0}>0$ and as $\widehat{\theta} \widehat{H}_{7} \neq 0$ and $\widehat{B}_{3}=\widehat{\mathcal{G}}_{1}=0$ according to $[27]$ we obtain Picture $4.2(b)$ (equivalent to the portrait $\left.V u l_{2}\right)$. So we obtain the configuration $\$, c ; N \quad[(0,1,0,1,0,2),(0,0,-1,-2,0,-1)]$. 3.2.2.2. Admit finally $\mu_{0}=0$, i.e. considering (65) and the condition $\mathcal{T}_{2} \neq 0$ we obtain $c=1$. Then for system (64) we obtain

$$
\begin{gathered}
\eta=-16<0, \quad \mu_{0}=\mu_{1}=0, \quad \mu_{2}=2\left(x^{2}+y^{2}\right), \quad \widehat{B}_{3}=\widehat{\mathcal{G}}_{1}=0, \\
\widetilde{K}=0, \quad \widehat{\theta}=0, \quad \widetilde{N}=4(x+y)^{2} \neq 0, \quad \widehat{H}_{7}=-32 .
\end{gathered}
$$

By [27] we get Picture 4.27(b) were two finite points have collided with the complex infinite points. This picture is equivalent to the phase portrait $V u l_{2}$ and we have the configuration $\$, c ; N, \quad[(0,1,0,1,0,1),(0,0,-1,-1,0,-1)]$. 
3.2.3. The subcase $\mathcal{T}_{3}=\mathcal{T}_{2}=0, \sigma \neq 0$. For systems (54) we have

$$
\mathcal{T}_{3}=-8\left(a^{3} c-k m^{3}\right), \quad \mathcal{T}_{2}=4\left(a^{3} c+3 a c k m-a^{2} m^{2}+k m^{3}\right), \quad \sigma=2(a x-m y) .
$$

So the condition $\sigma \neq 0$ implies $a^{2}+m^{2} \neq 0$ and we may assume $m \neq 0$ due to the change $(x, y, t, a, c, k, m) \mapsto(y, x,-t, m, k, c, a)$. Moreover we may consider $m=1$ due to the rescaling $y \rightarrow y / m$. Therefore the condition $\mathcal{T}_{3}=0$ gives $k=a^{3} c$ and then we get

$$
\begin{gathered}
\mathcal{T}_{2}=4 a^{2}(1+a c)(3 a c-1), \quad \mu_{0}=a^{2}(a c-1)^{2}(1+a c)^{2}, \\
\mu_{1}=2 a(1-a c)(1+a c)(a x-y), \quad \mu_{2}=(1+a c)\left[a^{2} x^{2}+3 a(a c-1) x y+y^{2}\right]
\end{gathered}
$$

3.2.3.1. Assume first $\mu_{0} \neq 0$. Then $a \neq 0$ and we may assume $a=1$ due to the change $x \rightarrow x / a$ and $c \rightarrow c / a$. Therefore as $\mu_{0} \neq 0$ the condition $\mathcal{T}_{2}=0$ implies $c=1 / 3$. This leads to the system

$$
\dot{x}=x+x^{2}+y^{2} / 3, \quad \dot{y}=-y-x^{2} / 3-y^{2},
$$

for which we calculate:

$$
\begin{gathered}
\mathcal{T}_{i}=0(i=1,2,3,4), \quad \sigma=2(x-y), \quad \mathbf{D}=\mathbf{T}=\mathbf{P}=0, \quad \mu_{0}=64 / 81, \\
\mathbf{R}=256 / 243(x-y)^{2}, \quad E_{3}=-16 / 81, \quad G_{10}=0, \quad \widehat{H}_{7}=-32 / 9, \\
\eta=\kappa=0, \quad \widetilde{M}=\widehat{B}_{3}=0, \quad C_{2}=(x+y)^{3} / 3, \quad \widehat{\theta}=-512 / 81 .
\end{gathered}
$$

Considering these values of the invariant polynomials according to Table 4 the systems above possess one triple and one simple real singularities (and the last one is an integrable saddle). Moreover by [3, Table 1, row 88] besides the saddle system (67) possesses one elliptic saddle. Due to $\widetilde{M}=0$ at infinity we have a triple point which is a node. This leads to the configuration $\$, \widehat{e s}_{(3)},\left(\begin{array}{l}0 \\ 3\end{array}\right) N$.

On the other hand as $\mu_{0}>0$ and $\widehat{\theta} \widehat{H}_{7} \neq 0$ according to [27] the phase portrait of this system is given by Picture 4.45(a) (equivalent to Portrait 35).

3.2.3.2. Suppose now $\mu_{0}=0$. We observe that this condition implies $\mu_{1}=0$ and we shall examine two cases: $\mu_{2} \neq 0$ and $\mu_{2}=0$.

3.2.3.2.1. If $\mu_{2} \neq 0$ then $(1+a c) \neq 0$ and hence the condition $\mathcal{T}_{2}=\mu_{0}=0$ yields $a=0$. So we get the family of systems

$$
\dot{x}=x+c y^{2}, \quad \dot{y}=-y-y^{2},
$$

for which calculations yield:

$$
\begin{gathered}
\sigma=-2 y, \quad \eta=\kappa=\kappa_{1}=0=G_{8}, \quad \mu_{0}=\mu_{1}=0, \quad \mu_{2}=y^{2}=F_{2}, \quad \mathbf{D}=\mathbf{R}=0, \\
\mathbf{P}=y^{4}, \quad \mathbf{U}=y^{4}(x-c y)^{2}, \quad \tilde{L}=8 y^{2}, \quad \widetilde{M}=-8 y^{2}, \quad D_{2}=-2 y, \quad K_{2}=96 y^{2} .
\end{gathered}
$$

Considering these values of invariant polynomials by Table 4 the systems above have two real finite singularities. And according to [3, Table 1, row 154] besides the saddle systems (69) possess a node.

On the other hand, due to the conditions $\eta=0, \widetilde{M} \neq 0, \mu_{0}=\mu_{1}=\kappa=\kappa_{1}=0, \tilde{L}>0$ and $K_{2}>0$ according to [25] at infinity we get Fig.19 (i.e. simple node and a saddle node of multiplicity four). So we arrive to the following configuration of singularities:

$\$, n ; N,\left(\begin{array}{l}2 \\ 2\end{array}\right) H P P-H P P \quad[(0,1,0,0,0,1),(0,0,-1,0,0,-1)]$

To determine the phase portrait following [28] we calculate

$$
\eta=0, \widetilde{M}=-8 y^{2}, \quad \mu_{0}=\widehat{B}_{3}=\widehat{\theta}=\widetilde{N}=\widehat{H}=\widehat{N}_{2}=0, \quad \widehat{D}=-y^{2}(x+c y), \widehat{N}_{1}=16 c y^{4} .
$$

According to [28] we get Picture 5.21 if $\widehat{N}_{1} \neq 0$ (i.e. $c \neq 0$ ) and and Picture 6.7 if $\widehat{N}_{1}=0$ (i.e. $c=0)$. It remains to note that these two phase portraits are topologically equivalent and here are represented by Portrait 43. 
3.2.3.2.2. Assume now $\mu_{2}=0$, then $(1+a c)=0$ and clearly $a \neq 0$. As it was mentioned above we may consider $a=1$ and therefore we get $c=-1$. This leads to the system

$$
\dot{x}=x+x^{2}-y^{2}, \quad \dot{y}=-y+x^{2}-y^{2},
$$

for which we have

$$
\begin{gathered}
\mu_{0}=\mu_{1}=\mu_{2}=\eta=\kappa=\tilde{L}=0, \quad \mu_{3}=(y-x)(x+y)^{2}=-K_{1}, \quad \kappa_{1}=-256, \\
\widetilde{M}=-32(x-y)^{2} \neq 0, \quad \widehat{B}_{3}=\widehat{\theta}=0, \quad \widetilde{N}=-4(x-y)^{2}, \quad \widehat{H}_{7}=-32 .
\end{gathered}
$$

So, the system above has only one finite singularity (the integrable saddle) and by [25] at infinity we get Fig. 9: $\overline{\left(\begin{array}{l}1 \\ 2\end{array}\right)} N, \overline{\left(\begin{array}{l}1 \\ 2\end{array}\right)} P E P-H$. So we get the configuration

$$
\$ ; \overline{\left(\begin{array}{l}
1 \\
2
\end{array}\right)} N, \overline{\left(\begin{array}{l}
1 \\
2
\end{array}\right)} P E P-H \quad[(0,1,0,1,0,-1),(0,0,-1,1,0,-1)] .
$$

On the other hand as for this system we have $\mu_{3} K_{1}<0$ according to [27] its phase portrait corresponds to Picture 4.35(b) which is topologically equivalent to $\mathrm{Ham}_{19}$.

3.2.4. The subcase $\sigma=0$ : Hamiltonian systems. For systems (54) the condition $\sigma=2(a x-$ $m y)=0$ gives $a=m=0$ and we arrive to the family of systems

$$
\dot{x}=x+c y^{2}, \quad \dot{y}=-y-k x^{2}, \quad c^{2}+k^{2} \neq 0 .
$$

For these systems we calculate

$$
\mu_{0}=c^{2} k^{2}, \quad \eta=-27 c^{2} k^{2}, \quad \widehat{\theta}=64 c^{2} k^{2}, \quad \widehat{B}_{3}=\widehat{\mathcal{G}}_{1}=0, \quad \widetilde{M}=72 c k x y=9 \widetilde{N}, \quad \widehat{H}_{7}=-32 c k
$$

3.2.4.1. Assume first $\mu_{0} \neq 0$. Then $c k \neq 0$ and we may assume $c=k=1$ due to the rescaling $(x, y) \mapsto\left(c^{-1 / 3} k^{-2 / 3} x, c^{-2 / 3} k^{-1 / 3} y\right)$. Herein for this system we have $\mathbf{D}=1296>0$ and as $\mu_{0} \neq 0$ according to Theorem 2 (see the statement $\left(f_{5}\right),[\alpha]$ ) we obtain an integrable saddle and a center (other two finite singularities being complex). Taking into account the condition $\eta<0$ at infinity we get a simple node and we arrive to the configuration

$\$, c ; N \quad[(0,1,0,0,0,1),(0,0,-1,-1,0,0)]$. On the other hand due to $\mu_{0}>0$ and $\eta<0$ according to [27] the phase portrait of this system is given by Picture 4.2(b) (topologically equivalent to $V u l_{2}$ ).

3.2.4.2. Suppose now $\mu_{0}=0$. Then $c k=0$ and $c^{2}+k^{2} \neq 0$ (otherwise we get a linear system). So without loss of generality we may assume $k=0$ due to the change $(x, y, t, c, k) \mapsto$ $(y, x,-t, k, c)$ and $c=1$ (due to a rescaling). We again obtain a single system for which we have

$$
\mu_{0}=\mu_{1}=\mu_{2}=\eta=\kappa=\kappa_{1}=\widetilde{M}=\widetilde{K}=\mathbf{R}=\mathbf{P}=0, \quad \mu_{3}=-y^{3}=-K_{1}, \quad \mathbf{U}=y^{6} .
$$

So in accordance with Table 4 this system has only one finite singularity (the integrable saddle) and by [25] at infinity we get Fig.33: $\left(\begin{array}{l}3 \\ 3\end{array}\right) P E P E P-P$. So we get the configuration

$$
\$ ;\left(\begin{array}{l}
3 \\
3
\end{array}\right) \text { PEPEP }-P \quad[(0,1,0,0,0,1),(0,0,-1,0,0,0)] \text {. }
$$

It remains to observe that in this case we have

$$
\eta=\widetilde{M}=\widetilde{N}=\widehat{B}_{3}=D_{1}=0, \quad \widehat{N}_{3}=-6 y^{3} \neq 0
$$

and by [28] the phase portrait of this system corresponds to Picture 5.26 which is equivalent to $\mathrm{Ham}_{18}$.

All the cases were examined. To complete the proof of the Main Theorem it remains to examine the intersection of the invariant conditions and the respective configurations obtained in two disjoint cases given by $b^{2}+l^{2} \neq 0$ and $b=l=0$ and to convince ourself that this leads to the respective conditions provided by Tables 1 and 2 .

\section{REFERENCES}

[1] J. C. Artés, R. Koolj, And J. Llibre, Structuraly stable quadratic vector fields, Mem. Am. Math. Soc. 134(639), 1998.

[2] J. C. Artés, J. Llibre And D. Schlomiuk, The geometry of quadratic dufferential systems with a weak focus of second order, International J. of Bifurcation and Chaos, 16 (2006), 31273194.

[3] J. C. Artés, J. Llibre And N. I. Vulpe, Singular points of quadratic systems: A complete classification in the coefficient space $\mathbb{R}^{12}$, International J. of Bifurcation and Chaos 18 (2008), 313-362. 
[4] J. C. Artés, J. Llibre And N. I. Vulpe, When singular points determine quadratic systems, Electron. J. Differential Equations 2008, No. 82, 37 pp.

[5] J. C. Artés, J. Llibre And N. I. Vulpe, Complete geometric invariant study of two classes of quadratic systems, Preprint 36, Barcelona, 2010, 28 pp.

[6] V.A. Baltag And N.I. Vulpe, The number and multiplicity of the singular points of the quadratic system, Dokl. Akad. Nauk. 323 (1992), 9-12 (Russian), Russian Acad. Sci. Dokl. Math. 45 (1993), 235-238 (English).

[7] V.A. Baltag And N.I. Vulpe, Affine-invariant conditions for determining the number and multiplicity of singular points of quadratic differential systems, Izv. Akad. Nauk Respub. Moldova Mat. 1993, no. 1, 39-48

[8] N.N. BAutin, On the number of limit cycles which appear with the variation of coefficients from an equilibrium point of focus or center type, Math. USSR-Sb. 100 (1954), 397-413; Translations Amer. Math. Soc. 1 (1962), 396-413.

[9] D. Bularas, Iu. Calin. L. Timochouk and N. Vulpe, T-comitants of quadratic systems: A study via the translation invariants, Delft University of Technology, Faculty of Technical Mathematics and Informatics, Report no. 96-90, 1996; (URL: ftp://ftp.its.tudelft.nl/publications/techreports/1996/DUT-TWI-96- 90.ps.gz).

[10] CAI, Sui Lin, The weak saddle and separatrix cycle of a quadratic system, Acta Math. Sinica, 30(1987), No.4, 553-559 (Chinese).

[11] J. Chavarriga, H. Giacomini, J. Giné and J. Llibre, On the integrability of two-dimensional flows, J. Differential Equations 157 (1999), 163-182

[12] W.A. Coppel, A Survey of Quadratic Systems, J. Differential Equations 2 (1966), $293-304$.

[13] F. Dumortier, J. Llibre And J. C. Artés, Qualitative Theory of Planar Differential Systems, Universitext, Springer-Verlag, New York-Berlin, ISBN: 3-540-32893-9 (2008).

[14] P. Joyal, C. Rousseau, Saddle Quantities and Applications. J. Differential Equations 78 (1989), No. 2 , 374-399.

[15] M.A. Liapunov Problème général de la stabilité du mouvement, Ann. of Math. Stud. 17, Princeton University Press, 1947.

[16] J. LlibRe AND D. Schlomiuk, The geometry of quadratic differential systems with a weak focus of third order, Canadian J. Math. 56 (2004), 310-343.

[17] R. Moussu, Une démonstration d'un théorème de Lyapunov-Poincaré, Astérisque 98-99 (1982), 216-223.

[18] P.J. Olver, Classical Invariant Theory, London Mathematical Society student texts: 44, Cambridge University Press, 1999.

[19] J. Pal And D. Schlomiuk, On the geometry in the neighborhood of infinity of quadratic differential phase portraits with a weak focus, Qualitative Theory of Dynamical Systems 2 (2001), 1-43.

[20] H. Poincaré, Mémoire sur les courbes définies par les équations différentielles, J. Math. Pure Appl. (4) 1 (1885) 167-244.

[21] H. Poincaré, Sur l'intégration des équations différentielles du premier ordre et du premier degré I and II, Rendiconti del circolo matematico di Palermo 5 (1891), 161-191; 11 (1897), 193-239.

[22] J.W. REYN, A bibliography of the qualitative theory of quadratic systems of differential equations in the plane, Delf University of Technology, http://ta.twi.tudelft.nl/DV/Staff/J.W.Reyn.html, 1997.

[23] R. Roussarie, Bifurcation of planar vector fields and Hilbert's sixteenth problem, Progress in Mathematics, Vol. 164, Birkhäuser Verlag, Basel, 1998.

[24] D. Schlomiuk, N. Vulpe, Planar quadratic differential systems with invariant straight lines of at least five total multiplicity, Qualitative Theory of Dynamical Systems, 5 (2004), 135-194.

[25] D. Schlomiuk, N. Vulpe, Geometry of quadratic differential systems in the neighborhood of infinity. J. Differential Equations, 2005, 215, 357-400.

[26] D. Schlomiuk, N. Vulpe, Planar quadratic differential systems with invariant straight lines of total multiplicity four, Nonlinear Anal., 2008, 68, No. 4, 681-715

[27] D. Schlomiuk, N. Vulpe, Integrals and phase portraits of planar quadratic differential systems with invariant lines of total multiplicity four, Bull. of Acad. of Sci. of Moldova. Mathematics, No. 1(56), 2008, $27-83$.

[28] D. Schlomiuk, N. Vulpe, Integrals and phase portraits of planar quadratic differential systems with invariant lines of at least five total multiplicity, Rocky Mountain J. Math. 38 (2008), 2015-2076.

[29] D. Schlomiuk, N. Vulpe, The full study of planar quadratic differential systems possessing a line of singularities at infinity, J. Dynam. Differential Equations 20 (2008), 737-775.

[30] D. Schlomiuk, N. Vulpe Global classification of the planar Lotka-Volterra differential systems according to their configurations of invariant straight lines, J. Fixed Point Theory Appl. 8 (2010), 177-245

[31] N. I. VulPE, Affine-invariant conditions for the topological discrimination of quadratic systems with a center, Differential Equations 19 (1983), 273-280

[32] N. VulPE, Characterization of the finite weak singularities of quadratic systems via invariant theory. Preprint 23, Barcelona, 2010, 42 pp.

[33] Ye YAnqIAn And others, Theory of Limit Cycles,Transl. Math. Monographs 66, Amer. Math. Soc., Providence, 1984.

[34] Ye YANQIAN, Qualitative Theory of Polynomial Differential Systems, Shanghai Scientific \& Technical Publishers, Shanghai, 1995 (in Chinese). 
1 Departament de Matemàtiques, Universitat Autònoma de Barcelona, 08193 Bellaterra, Barcelona, SPAIN

E-mail address: artes@mat.uab.cat, jllibre@mat.uab.cat

${ }^{2}$ Institute of Mathematics and Computer Science, Academy of Science of Moldova, 5 Academiei Str, Chişinău, MD-2028, Moldova

E-mail address: nvulpe@gmail.com 